\title{
Quantum and Information Thermodynamics: A Unifying Framework Based on Repeated Interactions
}

\author{
Philipp Strasberg, ${ }^{\dagger}$ Gernot Schaller, and Tobias Brandes ${ }^{*}$ \\ Institut für Theoretische Physik, Technische Universität Berlin, \\ Hardenbergstraße 36, D-10623 Berlin, Germany \\ Massimiliano Esposito \\ Complex Systems and Statistical Mechanics, Physics and Materials Science, \\ University of Luxembourg, L-1511 Luxembourg, Luxembourg
}

(Received 9 October 2016; revised manuscript received 17 January 2017; published 7 April 2017)

\begin{abstract}
We expand the standard thermodynamic framework of a system coupled to a thermal reservoir by considering a stream of independently prepared units repeatedly put into contact with the system. These units can be in any nonequilibrium state and interact with the system with an arbitrary strength and duration. We show that this stream constitutes an effective resource of nonequilibrium free energy, and we identify the conditions under which it behaves as a heat, work, or information reservoir. We also show that this setup provides a natural framework to analyze information erasure ("Landauer's principle") and feedback-controlled systems ("Maxwell's demon"). In the limit of a short system-unit interaction time, we further demonstrate that this setup can be used to provide a thermodynamically sound interpretation to many effective master equations. We discuss how nonautonomously driven systems, micromasers, lasing without inversion and the electronic Maxwell demon can be thermodynamically analyzed within our framework. While the present framework accounts for quantum features (e.g., squeezing, entanglement, coherence), we also show that quantum resources do not offer any advantage compared to classical ones in terms of the maximum extractable work.
\end{abstract}

DOI: 10.1103/PhysRevX.7.021003

Subject Areas: Mesoscopics, Quantum Physics, Statistical Physics

\section{INTRODUCTION}

Thermodynamics was traditionally designed to understand the laws that govern the behavior of macroscopic systems at equilibrium in terms of a few macroscopic variables (e.g., temperature, pressure, chemical potential, energy, volume, particle number, etc.) containing very limited information about the microscopic state of the system. Remarkable progress has been made over the last decades to understand under which conditions the laws of thermodynamics emerge for small-scale systems where quantum and stochastic effects dominate and which are usually far away from thermal equilibrium. This includes a consistent thermodynamic framework for driven systems weakly coupled to large and fast thermal reservoirs, which are described by a microscopically derived (quantum) master equation (ME) [1-4]. Such MEs can also be used as a basis to establish universal fluctuation relations which replace the traditional second law formulated as an

\footnotetext{
*Deceased.

†phist@physik.tu-berlin.de
}

Published by the American Physical Society under the terms of the Creative Commons Attribution 3.0 License. Further distribution of this work must maintain attribution to the author(s) and the published article's title, journal citation, and DOI. inequality by an exact symmetry that fluctuations must satisfy arbitrarily far from equilibrium [5-9]. This theory has been very successful to study small systems in a large variety of fields ranging from biophysics to electronics, and many of its predictions have been verified experimentally [10-16].

Yet, many situations encountered nowadays force us to go beyond the setup of driven systems weakly coupled to thermal reservoirs. Notable examples include the thermodynamic description of computation and information processing using feedback controls ("Maxwell demons") where different members of the statistical ensemble undergo different drivings [17-21], systems interacting with reservoirs prepared in nonequilibrium states [22-28] or nonGibbsian equilibrium states [29], and systems described by non-Hermitian dynamics [30].

In this paper, we extend the traditional framework of thermodynamics by considering a system which, in addition to being in contact with a thermal reservoir, interacts with a stream of external systems which we call "units." Each independently prepared unit interacts for a certain time with the system before being replaced by another one, and no additional assumption about the state of the units nor the system-unit interaction is required. In the most general picture, this stream of units will be shown to constitute a resource of nonequilibrium free energy modifying the 
traditional energetic and entropic balances. We will study the limits in which the stream of units effectively reproduces the effect of a heat, work, or information reservoir. We will also explore limits giving rise to an effective closed dynamics for the system, which still allows for a consistent thermodynamic description. We will focus on the ensembleaveraged description and not on fluctuations.

The benefit of our generalized thermodynamic framework is that it provides a unified perspective and encompasses many previously considered setups. In modern physics, such setups have probably first been used in quantum optics, theoretically as well as experimentally, to model a maser in which a stream of atoms is injected into a cavity in order to macroscopically populate it [31-33]. Such setups have also been used to stabilize photon number states via measurement-based quantum feedback control [34]. In theoretical works, an "information reservoir" made of a stream of bits was proposed to extract work from a single heat reservoir $[35,36]$, a picture that also closely resembles a Turing machine [17,37]. The setup is also close to resource theoretic formulations of thermodynamics, in which one investigates which system transformations are possible given a set of freely available states and resources (all other states) [38,39]. Further analogies can be drawn with biomolecular motors or enzymes [11] which manipulate, e.g., nucleic acids (units) on a DNA strand, or with scattering theory where incoming and outgoing wave packets (units) interact for a short time with the scatterer (the system) [40,41].

\section{A. Outline}

The structure of the paper and some of its main results are summarized in Fig. 1. We start very generally in Sec. II by considering two interacting systems, and we review under what conditions the laws of thermodynamics can be established if one of the systems is initially in a thermal state and plays the role of the reservoir while the other is the driven system of interest. Besides establishing notation, this section also sets the basis for Sec. III, where the system now also interacts with an external stream of units. Generalized laws of thermodynamics are established which show that the stream of units effectively constitutes a resource of nonequilibrium free energy. We then consider various limiting cases in Sec. IV, where the stream of units respectively behaves as a heat, work, and information reservoir. Furthermore, Landauer's principle is derived, as well as a quantum version of the second law of thermodynamics under feedback control. We go one step further in Sec. V by considering scenarios leading to a closed reduced dynamics for the system when tracing out the units, but where one still retains a consistent thermodynamic description. More specifically, we consider the limit of infinitesimally short interactions which are either Poissonian or regularly distributed and which lead to effective MEs. We also analyze the limit where the units effectively generate a time-dependent Hamiltonian for the system. Specific models, apparently unrelated in the literature, are then analyzed within our unifying framework in the subsequent section (Sec. VI). These include the Mandal-Jarzynski engine, the micromaser, lasing without inversion where work is extracted from quantum coherence, and the electronic Maxwell demon. We finally close the paper with Sec. VII, where we first show that our generalized second law of thermodynamics does not conflict with the traditional Kelvin-Planck statement of

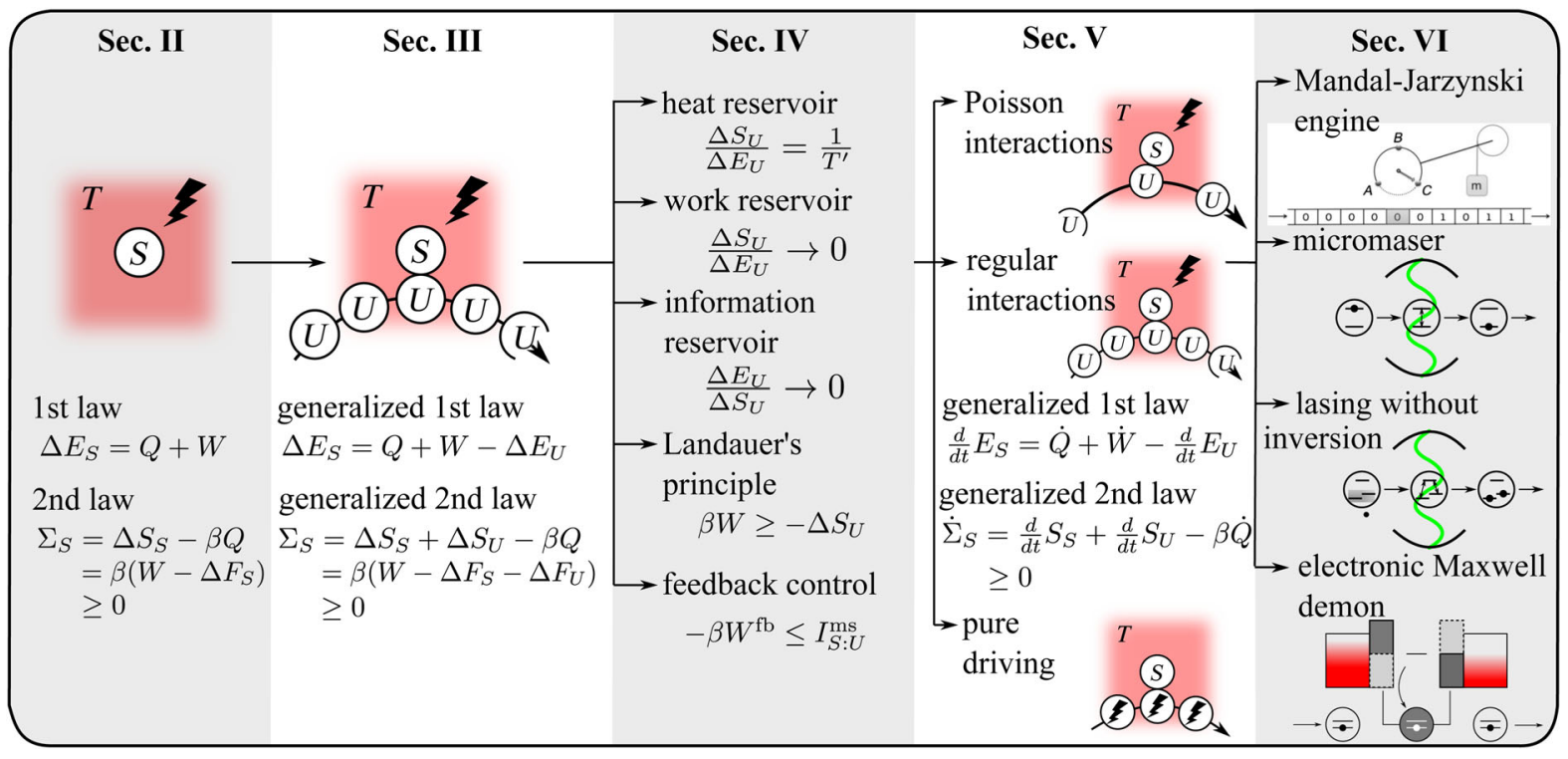

FIG. 1. Overview of the structure of the article covering its main results and applications. Here and in the following, $E_{S, U}$, $S_{S, U}$, and $F_{S, U}$ denote the energy, entropy, and nonequilibrium free energy of the system $(S)$ or unit $(U)$. $Q$ is the heat flowing from the reservoir at inverse temperature $\beta=T^{-1}\left(k_{B} \equiv 1\right)$, and $W$ is the work done on the system. Furthermore, $\Sigma_{S}$ denotes the entropy production and $I_{S}: U$ the mutual information between the system and unit. The picture of the Mandal-Jarzynski engine was taken from Ref. [35]. 
the second law; we then prove that quantum thermodynamics offers a priori no advantages compared to classical thermodynamics within our framework; and we finally give a short summary and outlook.

\section{ENERGY AND ENTROPY BALANCE OF AN OPEN QUANTUM SYSTEM}

\section{A. Two interacting systems}

To introduce important concepts and notation, we start by considering two interacting systems $X$ and $Y$, which are initially (at time $t=0$ ) decorrelated, i.e.,

$$
\rho_{X Y}(0)=\rho_{X}(0) \otimes \rho_{Y}(0) \equiv \rho_{X}(0) \rho_{Y}(0) .
$$

Here, $\rho_{X Y}$ denotes the density operator of the compound system $X$ and $Y$, whereas $\rho_{X}\left(\rho_{Y}\right)$ describes the reduced state of $X(Y)$. In order to make a statement about the first law of thermodynamics, we associate a Hamiltonian to the setup, which we decompose as

$$
\begin{aligned}
H_{\mathrm{tot}}(t) & =H_{X}(t) \otimes \mathbf{1}_{Y}+\mathbf{1}_{X} \otimes H_{Y}(t)+H_{X Y}(t) \\
& \equiv H_{X}(t)+H_{Y}(t)+H_{X Y}(t) .
\end{aligned}
$$

Here, $\mathbf{1}_{X}\left(\mathbf{1}_{Y}\right)$ denotes the identity on the Hilbert space of system $X(Y)$, which we usually suppress to simplify notation, and $H_{X Y}(t)$ describes the interaction between systems $X$ and $Y$. Furthermore, all terms can, in principle, be explicitly time dependent.

The time evolution of the compound system is governed by the Liouville-von Neumann equation $d_{t} \rho_{X Y}(t)=-i\left[H_{\text {tot }}(t), \rho_{X Y}(t)\right](\hbar \equiv 1$ throughout the text $)$. Introducing the unitary time evolution operator $U_{t} \equiv$ $\mathcal{T}_{+} \exp \left[-i \int_{0}^{t} d s H_{\text {tot }}(s)\right]$ (where $\mathcal{T}_{+}$denotes the time ordering operator), the state of the compound system at time $\tau$ is given by

$$
\rho_{X Y}(\tau)=U_{\tau} \rho_{X}(0) \rho_{Y}(0) U_{\tau}^{\dagger},
$$

which is, in general, correlated.

To obtain the reduced state of system $X$, we must trace over system $Y$. Using the spectral decomposition of the initial density matrix of system $Y, \rho_{Y}(0)=\sum_{l} p_{l}|l\rangle_{Y}\langle l|$, we arrive at

$$
\begin{aligned}
\rho_{X}(\tau) & =\operatorname{tr}_{Y}\left\{\rho_{X Y}(\tau)\right\} \\
& =\sum_{k l} T_{k l} \rho_{X}(0) T_{k l}^{\dagger} \equiv \Phi_{X}(\tau) \rho_{X}(0),
\end{aligned}
$$

where $T_{k l} \equiv \sqrt{p_{l}}\left\langle k\left|U_{\tau}\right| l\right\rangle_{Y}$. The $T_{k l}$ are still operators in the Hilbert space of system $X$, and they fulfill the completeness relation

$$
\sum_{k l} T_{k l}^{\dagger} T_{k l}=\mathbf{1}_{X}
$$

The map $\Phi_{X}(\tau)$ in Eq. (4) is known as a Kraus map or quantum operation, and it is the most general map (preserving the density matrix properties) for a quantum system which was initially uncorrelated [42-45]. We note that the representation of $\Phi_{X}$ in terms of the Kraus operators $T_{k l}$ is not unique.

The energy of the compound system at any point in time is $E_{X Y}(t) \equiv \operatorname{tr}_{X Y}\left\{H_{\text {tot }}(t) \rho_{X Y}(t)\right\}$. Because the compound system is isolated, the dynamics is unitary and the energy change is solely due to the time dependence in the Hamiltonian and can thus be identified as work,

$$
d_{t} E_{X Y}(t)=\operatorname{tr}_{X Y}\left\{\rho_{X Y}(t) d_{t} H_{\mathrm{tot}}(t)\right\} \equiv \dot{W}(t) .
$$

The rate of work injection $\dot{W}$ is positive if it increases the energy of the compound system. Equation (6) is the first law of thermodynamics for an isolated system.

We now consider the entropy balance by defining the von Neumann entropy of a system $X$, which we interpret as a measure for our lack of knowledge about the state $\rho_{X}$, as usual, by

$$
S_{X} \equiv-\operatorname{tr}_{X}\left\{\rho_{X} \ln \rho_{X}\right\}
$$

Because the joint von Neumann entropy $S_{X Y}(t) \equiv$ $-\operatorname{tr}_{X Y}\left\{\rho_{X Y}(t) \ln \rho_{X Y}(t)\right\}$ does not change under unitary time evolution, we have $S_{X Y}(\tau)=S_{X Y}(0)$. We further introduce the non-negative (quantum) mutual information

$$
I_{X: Y}(t) \equiv S_{X}(t)+S_{Y}(t)-S_{X Y}(t) \geq 0,
$$

which measures the amount of correlations shared between $X$ and $Y$ [43]. For the initially decorrelated state (1), we have $S_{X Y}(0)=S_{X}(0)+S_{Y}(0)$. Hence, in terms of the mutual information, we can write that

$$
I_{X: Y}(\tau)=\Delta S_{X}(\tau)+\Delta S_{Y}(\tau) \geq 0 .
$$

Thus, the mutual information tells us how the sum of the marginal entropies $\Delta S_{X}(\tau) \equiv S_{X}(\tau)-S_{X}(0)$ and $\Delta S_{Y}(\tau) \equiv$ $S_{Y}(\tau)-S_{Y}(0)$ can change.

Introducing the relative entropy between two density matrices $\rho$ and $\sigma$ [43],

$$
D(\rho \| \sigma) \equiv \operatorname{tr}\{\rho(\ln \rho-\ln \sigma)\} \geq 0,
$$

which is non-negative by Klein's inequality, the mutual information can also be written as

$$
I_{X: Y}(t)=D\left[\rho_{X Y}(t) \| \rho_{X}(t) \rho_{Y}(t)\right] \geq 0 .
$$

By measuring the local entropy changes in $X$ and $Y$, the mutual information is therefore also a measure of the 
information lost when disregarding the correlation established over time $t$ between $X$ and $Y$ while keeping full knowledge of $X$ and of $Y$ separate in the description. Note that relative entropy is not symmetric, i.e., $D(\rho \| \sigma) \neq$ $D(\sigma \| \rho)$ in general, but mutual information fulfills $I_{X: Y}=$ $I_{Y: X}$. Furthermore, it is important to mention that the action of any Kraus map $\Phi$ can never increase the relative entropy $[44,46]$, i.e.,

$$
D(\Phi \rho \| \Phi \sigma) \leq D(\rho \| \sigma) .
$$

\section{B. System coupled to a thermal reservoir}

To make further contact with thermodynamics, we now consider the case where the system $Y$ is supposed to play the role of a thermal reservoir. For this purpose, we relabel $Y$ by $R$ and make the two assumptions that the Hamiltonian $H_{R}$ is time independent and that the initial state of the reservoir is thermal:

$$
\rho_{R}(0)=\rho_{\beta}^{R} \equiv \frac{e^{-\beta H_{R}}}{Z_{R}}, \quad Z_{R}=\operatorname{tr}\left(e^{-\beta H_{R}}\right) .
$$

Similar treatments were presented, e.g., in Refs. [47-50].

Following the rational of Eq. (6), the energy change in the total system is identified as the work done by the external time-dependent driving on the system,

$$
\begin{aligned}
d_{t} E_{X Y}(t) & =\operatorname{tr}_{X R}\left\{\rho_{X R}(t) d_{t}\left[H_{X}(t)+H_{X R}(t)\right]\right\} \\
& \equiv \dot{W}(t) .
\end{aligned}
$$

The energy flowing out of the reservoir is in turn identified as the heat flow into the system $X$ at time $t$ (positive if it increases the system energy),

$$
\dot{Q}(t) \equiv-\operatorname{tr}_{R}\left\{H_{R} d_{t} \rho_{R}(t)\right\} .
$$

Consequently, the internal energy of the system $X$ is identified as

$$
E_{X}(t) \equiv \operatorname{tr}_{X R}\left\{\left[H_{X}(t)+H_{X R}(t)\right] \rho_{X R}(t)\right\}
$$

so that

$$
d_{t} E_{X}(t)=\dot{W}(t)+\dot{Q}(t)
$$

This constitutes the first law of thermodynamics for a closed system. We use the conventional terminology of thermodynamics where a system exchanging only energy (but not matter) with a reservoir is called "closed," though one would rather call it "open" from the perspective of open quantum system theory. An open system in the thermodynamic sense (also exchanging matter with its environment) can be considered by introducing a chemical potential for reservoir $R$, which is then described by an initial grand canonical equilibrium state. Integrating the first law over an interval $[0, \tau]$ gives

$$
\Delta E_{X}(\tau)=E_{X}(\tau)-E_{X}(0)=W(\tau)+Q(\tau)
$$

with

$$
\begin{aligned}
W(\tau) & \equiv \int_{0}^{\tau} d t \dot{W}(t)=E_{X R}(\tau)-E_{X R}(0), \\
Q(\tau) & \equiv \int_{0}^{\tau} d t \dot{Q}(t)=-\operatorname{tr}_{R}\left\{H_{R}\left[\rho_{R}(\tau)-\rho_{R}(0)\right]\right\} .
\end{aligned}
$$

By analogy with the second law of phenomenological nonequilibrium thermodynamics [51] which states that the non-negative entropy production characterizing the irreversibility of a process is given by the sum of the entropy change in the system and in the (macroscopic ideal and always equilibrated) reservoir, we follow Ref. [47] and define entropy production as

$$
\Sigma(\tau) \equiv \Delta S_{X}(\tau)-\beta Q(\tau)
$$

Since the initial reservoir state (13) is thermal, the nonnegativity of $\Sigma$ can be shown by noting the identities [47]

$$
\begin{aligned}
\Sigma(\tau) & =D\left[\rho_{X R}(\tau) \| \rho_{X}(\tau) \otimes \rho_{\beta}^{R}\right] \\
& =D\left[\rho_{R}(\tau) \| \rho_{\beta}^{R}\right]+I_{X: R}(\tau) \geq 0 .
\end{aligned}
$$

It relies on the assumption that the initial total system state is decorrelated. We emphasize that $\Sigma(\tau) \geq 0$ holds for any reservoir size and thus cannot be considered as strictly equivalent to the phenomenological second law of nonequilibrium thermodynamics. We also note that expression (21) provides interesting insight into the difference between the way in which we treated the reservoir $R$ in this section compared to the way in which we treated system $Y$ in the previous section: The entropy production not only measures the information lost in the correlations between the system and the reservoir via the mutual information $I_{X: R}$, it also measures the information lost in not knowing the state of the reservoir after the interaction via $D\left[\rho_{R}(\tau) \| \rho_{\beta}^{R}\right]$. This translates into the obvious fact in thermodynamics that one has no access to the state of the reservoir and that one only knows the energy that has flown in it as heat.

We define an ideal heat reservoir as a reservoir that remains close to thermal equilibrium during its interaction with the system, i.e., $\rho_{R}(\tau)=\rho_{\beta}^{R}+\epsilon \sigma_{R}$, where $\epsilon$ is a small parameter and $\operatorname{tr}_{R}\left(\sigma_{R}\right)=0$. Using the exact identity

$$
T D\left[\rho_{R}(\tau) \| \rho_{\beta}^{R}\right]=-Q(\tau)-T \Delta S_{R}(\tau) \geq 0,
$$

this means that the information lost by not knowing the reservoir state becomes negligible because $D\left[\rho_{R}(\tau) \| \rho_{\beta}^{R}\right]=$ $\mathcal{O}\left(\epsilon^{2}\right)$ [52]. Consequently, the entropy change in the 
reservoir can be solely expressed in terms of the heat flowing in it via Clausius equality, i.e., $\Delta S_{R}(\tau)=-\beta Q(\tau)$, where these two quantities are generically of first order in $\epsilon$ and only differ from each other to second order in $\epsilon$. Using Eq. (21), this also means that the entropy production due to an ideal heat reservoir coincides (to second order in $\epsilon$ ) with the lost mutual information between the system and the reservoir, i.e., $\Sigma(\tau)=I_{X: R}(\tau)$.

Finally, it is useful to introduce the concept of a nonequilibrium free energy. Following Refs. [47,53-55], we define

$$
F_{X}(t) \equiv E_{X}(t)-T S_{X}(t)
$$

where $T$ is the temperature of the initial reservoir attached to the system. Since it is fixed, $d F_{X}(t)$ is still an exact differential. Using this quantity, we can write the second law as

$$
\Sigma(\tau)=\beta\left[W(\tau)-\Delta F_{X}(\tau)\right] \geq 0 .
$$

Explicit processes where $\Sigma(\tau)$ can be made arbitrarily close to zero have been considered, e.g., in Refs. [49,55-58].

\section{Weak-coupling limit and master equations}

The results above provide a general way to formally derive the laws of thermodynamics as exact identities. Remarkably, these relations hold even if the reservoir $R$ is arbitrarily small and strongly influenced by the presence of the system. However, while the entropy production $\Sigma(\tau)$ is proven to be non-negative, its rate can be negative. Indeed, as finite-size quantum systems evolve quasiperiodically, the exact rate of entropy production must become negative at some time to restore the initial state. Furthermore, these identities are also of limited practical use because computing any particular expression requires solving the full Liouville-von Neumann dynamics for the joint system and reservoir.

The weak-coupling limit between the system and the reservoir circumvents these limitations and is of great practical relevance. It has been used for a long time to study quantum thermodynamics $[59,60]$ (see also Refs. [1,3] for recent reviews). Within this limit, the system does not perturb the reservoir over any relevant time scale, and it is further assumed that the reservoir behaves memoryless (i.e., Markovian). This allows us to close the equation of motion for the system density matrix $\rho_{X}(t)$. The resulting dynamics is called a (quantum) master equation (ME). In this limit, the general results of Sec. II B reduce to the well-known ME formulation of quantum thermodynamics [47], where all thermodynamic quantities can be expressed solely in terms of system operators.

More specifically, after applying the Born-Markovsecular approximation [1,2,5,59,61], which is usually justified in the weak-coupling limit, the ME can be put into the form [62]

$$
\begin{aligned}
d_{t} \rho_{X}(t) & =-i\left[H_{X}(t), \rho_{X}(t)\right]+\mathcal{L}_{\beta}(t) \rho_{X}(t) \\
& \equiv \mathcal{L}_{X}(t) \rho_{X}(t)
\end{aligned}
$$

where $\mathcal{L}_{\beta}(t)$ and $\mathcal{L}_{X}(t)$ denote superoperators that act linearly on the space of system operators. In order to derive Eq. (25), one also has to assume that the driving of $H_{X}(t)$ is slow compared to the relaxation time of the reservoir, though this does not imply that the driving must be adiabatic [63-65]. For a system-reservoir coupling of the form $H_{X R}=\sum_{k} A_{k} \otimes B_{k}$ with Hermitian system and reservoir operators $A_{k}$ and $B_{k}$, the superoperator $\mathcal{L}_{\beta}(t)$ reads (see, e.g., Sec. 3.3 in Ref. [61] for a microscopic derivation)

$$
\begin{aligned}
\mathcal{L}_{\beta}(t) \rho(t)= & \sum_{\omega} \sum_{k, \ell} \gamma_{k \ell}(\omega) \\
& \times\left(A_{\ell}(\omega) \rho(t) A_{k}^{\dagger}(\omega)-\frac{1}{2}\left\{A_{k}^{\dagger}(\omega) A_{\ell}(\omega), \rho(t)\right\}\right) .
\end{aligned}
$$

Here, $\left\{\omega=\epsilon-\epsilon^{\prime}\right\}$ denotes the set of transition frequencies of the Hamiltonian $H_{S}(t)=\sum_{\epsilon} \epsilon(t) \Pi_{\epsilon(t)}$ with instantaneous eigenenergies $\epsilon(t)$ and corresponding projection operators $\Pi_{\epsilon(t)}$. Omitting the explicit time dependence in the notation, $A_{k}(\omega)$ is defined as

$$
A_{k}(\omega) \equiv \sum_{\epsilon-\epsilon^{\prime}=\omega} \Pi_{\epsilon} A_{k} \Pi_{\epsilon^{\prime}} .
$$

Furthermore, the rate $\gamma_{k \ell}(\omega)$ is the Fourier-transformed reservoir correlation function

$$
\gamma_{k \ell}(\omega)=\int_{-\infty}^{\infty} d t e^{i \omega t} \operatorname{tr}_{R}\left\{B_{k}(t) B_{\ell}(0) \rho_{\beta}^{R}\right\}
$$

which can be shown to be non-negative [61]. The thermal generator (26) fulfills two important properties. First, it is of so-called Lindblad form $[69,70]$. This means that the time evolution of Eq. (25),

$$
\rho_{X}(\tau)=\mathcal{T}_{+} \exp \left[\int_{0}^{\tau} d s \mathcal{L}_{X}(s)\right] \rho_{X}(0)
$$

[or simply $\rho_{X}(\tau)=e^{\mathcal{L}_{X} \tau} \rho_{X}(0)$ if $\mathcal{L}_{X}$ is time independent], can be written as a Kraus map (4). Whereas each Lindblad ME defines a particular Kraus map, the inverse is not necessarily true; i.e., for a given Kraus map, we cannot associate a unique ME in Lindblad form. Second, and very important from the thermodynamic point of view, the rates satisfy the property of local detailed balance

$$
\gamma_{k \ell}(-\omega)=e^{-\beta \omega} \gamma_{\ell k}(\omega),
$$

which follows from the Kubo-Martin-Schwinger (KMS) relation of the reservoir correlation functions $[1,2,5,59,61]$. 
It also plays a crucial role to establish a consistent nonequilibrium thermodynamics for classical stochastic processes $[8,9]$. More importantly for us here, it implies that the steady state of the system corresponds to the canonical equilibrium (or Gibbs) state [61]:

$$
\mathcal{L}_{\beta}(t) \rho_{\beta}^{X}(t)=0, \quad \rho_{\beta}^{X}(t)=\frac{e^{-\beta H_{X}(t)}}{Z_{X}(t)} .
$$

Since the system-reservoir interaction is now negligible, the system energy reads $E_{X}(t) \equiv \operatorname{tr}\left\{H_{X}(t) \rho_{X}(t)\right\}$ and the first law takes the usual form,

$$
d_{t} E_{X}(t)=\dot{W}(t)+\dot{Q}(t),
$$

where the work rate on the system is given by

$$
\dot{W}(t)=\operatorname{tr}_{X}\left\{\rho_{X}(t) d_{t} H_{X}(t)\right\}
$$

and the heat flow into the system is caused by the dissipative part of the evolution,

$$
\begin{aligned}
\dot{Q}(t) & =\operatorname{tr}_{X}\left\{H_{X}(t) d_{t} \rho_{X}(t)\right\} \\
& =\operatorname{tr}_{X}\left\{H_{X}(t) \mathcal{L}_{X}(t) \rho_{X}(t)\right\} .
\end{aligned}
$$

The second law is now more stringent since it not only ensures the non-negativity of the entropy production but also of its rate,

$$
\dot{\Sigma}(t)=d_{t} S_{X}(t)-\beta \dot{Q}(t) \geq 0 .
$$

Mathematically, this result follows from Spohn's inequality [71]

$$
-\operatorname{tr}\left\{\left[\mathcal{L}_{X}(t) \rho_{X}(t)\right]\left[\ln \rho_{X}(t)-\ln \bar{\rho}_{X}(t)\right]\right\} \geq 0 .
$$

This is true for any superoperator $\mathcal{L}_{X}(t)$ of Lindblad form with steady state $\bar{\rho}_{X}(t)$, i.e., $\mathcal{L}_{X}(t) \bar{\rho}_{X}(t)=0$, and it corresponds to the differential version of Eq. (12). For the case considered here, we have $\bar{\rho}_{X}(t)=\rho_{\beta}^{X}(t)$ [see Eq. (31)], and Spohn's inequality gives, after a straightforward manipulation, Eq. (35).

\section{THERMODYNAMICS OF REPEATED INTERACTIONS}

\section{A. Idea}

Traditional thermodynamic setups consist of a system in contact with thermal reservoirs, as reviewed in the previous section. However, as motivated in the Introduction, many experimental (and theoretical) setups today do not fit into this picture, making use of much more structured resources. For instance, a micromaser (as reviewed in Sec. VI B) makes use of a stream of flying atoms prepared in nonequilibrium states interacting sequentially with a cavity. Another example is the measurement and subsequent manipulation of a device by an external observer ("Maxwell's demon"); see Secs. IV E and VID.

Although both examples are quite different, we can treat them within a unified framework by introducing a new form of "reservoir" which can be prepared in arbitrary nonequilibrium states. The basic setup consists of a stream of additional systems, which we generically call "units." These units are identically and independently prepared and interact one after another with the device (i.e., the system of interest) without ever coming back. This framework of repeated interactions provides sufficient structure to formulate a meaningful and tractable dynamics and thermodynamics, as we now demonstrate.

\section{B. Formal setup}

The system $X$ that we considered so far is split into two as $X=S \oplus U$, where $S$ denotes the system of interest whereas $U$ is an auxiliary system called the unit. We assume that this unit interacts with the system $S$ for a time $\tau^{\prime}$ and is afterwards decoupled from it and replaced at time $\tau>\tau^{\prime}$ by a new unit. This new unit interacts with $S$ from $\tau$ to $\tau+\tau^{\prime}$ before it is replaced by yet another new unit at $2 \tau$. The process is then repeated over and over. This means that the system is interacting sequentially with a stream of units $\left\{U_{n}\right\}$, where the index $n$ refers to the $n$th interaction taking place in the interval $[n \tau, n \tau+\tau)$ as sketched in Fig. 2.

The Hamiltonian $H_{X}(t)$ of the joint system and all units can be formally written as

$$
H_{X}(t)=H_{S}(t)+H_{U}+H_{S U}(t),
$$

where the system Hamiltonian $H_{S}(t)$ may or may not be time dependent. The Hamiltonian of the stream of (noninteracting) units can be written as a sum of (timeindependent) single-unit Hamiltonians $H_{U}^{(n)}$,

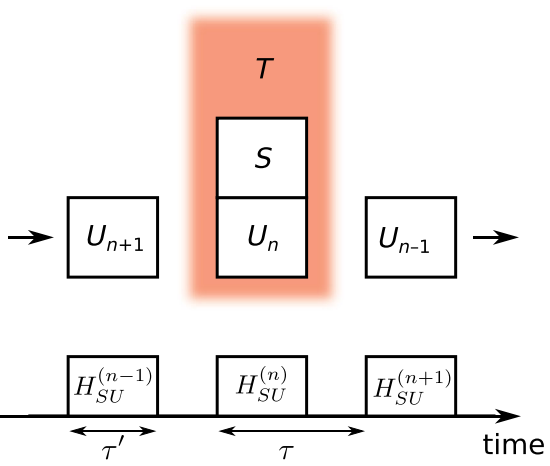

FIG. 2. Sketch of a stream of units interacting with a system in contact with a heat reservoir at inverse temperature $\beta$. The lower panel shows the switching on and off of the system-unit interaction as a function of time. Note that $\tau$ denotes the full interaction period, whereas the system and unit are only physically coupled during a time $\tau^{\prime}$. 


$$
H_{U}=\sum_{n} H_{U}^{(n)}
$$

and the system-unit interaction as

$$
H_{S U}(t)=\sum_{n} \Theta(t-n \tau) \Theta\left(n \tau+\tau^{\prime}-t\right) V_{S U}^{(n)}(t),
$$

where $V_{S U}^{(n)}(t)$ is an arbitrary interaction between the system and the $n$th unit. The Heaviside step function explicitly reads

$$
\Theta(t) \equiv \begin{cases}1 & \text { if } t \geq 0 \\ 0 & \text { if } t<0 .\end{cases}
$$

This means that the interaction between the system and the $n$th unit is switched on at time $t=n \tau$ and switched off at $t=n \tau+\tau^{\prime}$ with $0<\tau^{\prime}<\tau$ as explained above. During this interaction time, the system and unit are both coupled to the reservoir $R$. Their dynamics can be described exactly or by a ME of the form (25) in the weak-coupling limit. However, before or after the interaction with the system, when the unit is not in contact with the system, it will evolve freely (i.e., unitarily) with $H_{U}^{(n)}$, and its energy $E_{U}^{(n)}(t)=\operatorname{tr}_{U_{n}}\left\{H_{U}^{(n)} \rho_{U}^{(n)}(t)\right\}$ and entropy $S_{U}^{(n)}(t)=$ $-\operatorname{tr}_{U_{n}}\left\{\rho_{U}^{(n)}(t) \ln \rho_{U}^{(n)}(t)\right\}$ will remain constant.

Our setup is meant to model situations where an experimentalist can prepare independent units in any desired state. Therefore, a crucial but reasonable assumption that we use is that the incoming units are decorrelated (i.e., independently prepared) and that their statistical description is stationary in time; i.e., the density matrix of the incoming units fulfills $\rho_{U}^{(n)}(n \tau)=\rho_{U}^{(m)}(m \tau)$ for any $n, m$ [72]. We further assume that the interaction Hamiltonian $V_{S U}^{(n)}(t)$ always has the same form [of course, it acts on different unit Hilbert spaces, but for simplicity, we always denote it by $\left.V_{S U}(t)\right]$.

Our goal is to formulate thermodynamic laws for the system where one regards the stream of units as a nonequilibrium reservoir, and to understand to what extent the latter modifies the traditional thermodynamic laws. In the next section, we focus on one fixed interval, where the system interacts with a single unit only. For simplicity, we choose the interval $[0, \tau)$ and drop the index $n=0$. Section III D then discusses what happens if the system is repeatedly put into contact with subsequent units and whether one can expect the system to reach a stroboscopic steady state.

\section{Modified energy and entropy balance}

To obtain the first law of thermodynamics, we can either take Eq. (18) or integrate Eq. (32), where care has to be taken with the definition of the time interval to correctly capture boundary effects. Thus, we define the global change of system and unit energy as

$$
\Delta E_{S U} \equiv \lim _{\epsilon \searrow 0} \int_{-\epsilon}^{\tau-\epsilon} d t \frac{d E_{X}(t)}{d t}=\Delta E_{S}+\Delta E_{U}
$$

such that the interaction term does not contribute. Here, $\Delta E_{S}=\operatorname{tr}_{S}\left\{H_{S}(\tau) \rho_{S}(\tau)\right\}-\operatorname{tr}_{S}\left\{H_{S}(0) \rho_{S}(0)\right\}$ and analogously for $\Delta E_{U}$. Integrating the rate of work yields two terms:

$$
W \equiv \lim _{\epsilon \searrow 0} \int_{-\epsilon}^{\tau-\epsilon} d t \dot{W}(t)=W_{X}+W_{\mathrm{sw}}
$$

The first term is standard and results from the smooth time dependence of $H_{S}(t)$ during the full interval and of $V_{S U}(t)$ during the interaction, i.e.,

$$
\begin{aligned}
W_{X}= & \int_{0}^{\tau} d t \operatorname{tr}_{X}\left\{\rho_{X}(t) d_{t} H_{S}(t)\right\} \\
& +\lim _{\epsilon \searrow 0} \int_{\epsilon}^{\tau^{\prime}-\epsilon} d t^{\prime} \operatorname{tr}_{X}\left\{\rho_{X}(t) d_{t} V_{S U}(t)\right\}
\end{aligned}
$$

The second term, which we refer to as the switching work, is a boundary term resulting from the sudden on and off switching of the interaction, and it reads

$$
W_{\mathrm{sw}}=\operatorname{tr}_{X}\left\{V_{S U}(0) \rho_{X}(0)-V_{S U}\left(\tau^{\prime}\right) \rho_{X}\left(\tau^{\prime}\right)\right\} .
$$

Mathematically, it follows from $d_{t} \Theta(t)=\delta(t)$, where $\delta(t)$ is a Dirac delta distribution. Physically, we can interpret $W_{\text {sw }}$ as the work needed to pull the stream of units along the system (we assume that the other units that do not interact with the system move in a frictionless way). Finally, by integrating the heat flow [Eq. (19) or (34)], we get

$$
Q \equiv \lim _{\epsilon \searrow 0} \int_{-\epsilon}^{\tau-\epsilon} d t \dot{Q}(t),
$$

and the first law of thermodynamics takes the form

$$
\Delta E_{S}=W+Q-\Delta E_{U}
$$

Using the second law of thermodynamics, Eq. (20) or Eq. (35), as well as the factorization of the initial condition $\rho_{X}(0)=\rho_{S}(0) \rho_{U}(0)$, the entropy production of the system and unit during each interaction period reads

$$
\Sigma=\Delta S_{S}+\Delta S_{U}-I_{S: U}(\tau)-\beta Q \geq 0,
$$

where the unit entropy change $\Delta S_{U}$ and the final systemunit correlations $I_{S: U}(\tau)$ modify what would otherwise be the traditional second law for the system in contact with its reservoir. In view of our interpretation of Eq. (20), Eq. (47) describes the dissipation of the joint system $S U$ to the reservoir. The units and the system are thus treated on the same footing. However, since each unit only interacts once with the system, the mutual information that they 
established by interacting with the system is never used or recovered [73]. A more meaningful definition of entropy production for our setup, which accounts for these losses, is thus

$$
\Sigma_{S} \equiv \Delta S_{S}+\Delta S_{U}-\beta Q \geq I_{S: U}(\tau) \geq 0 .
$$

This entropy production not only measures the lost information as system-reservoir mutual information and as relative entropy between the nonequilibrium and equilibrium reservoir state after the interaction [compare with Eq. (21)], but it also accounts for the information lost as mutual information by the units that never come back. Obviously, $\Sigma_{S} \geq \Sigma$. In the special case where the coupling to the reservoir is switched off, $Q=0$ and $\Sigma=0$, the entropy production is solely given by the mutual information $\Sigma_{S}=I_{S: U}$ lost in our setup.

Using the first law (46) together with the definition of the nonequilibrium free energy (23) with respect to the reservoir temperature $\beta$, Eq. (48) can be rewritten as

$$
\Sigma_{S}=\beta\left(W-\Delta F_{S}-\Delta F_{U}\right) \geq I_{S: U}(\tau) \geq 0 .
$$

This form of the modified second law allows us to draw the important conclusion that the stream of units in its most general sense is nothing but a resource of nonequilibrium free energy.

\section{Steady-state regime}

We derived our modified laws of thermodynamics for an arbitrary initial system state over a single interaction interval. To treat many interaction intervals, we have to link the (thermo)dynamics between successive interaction intervals; i.e., the final system state $\rho_{S}(n \tau)$ of the $n$th interaction interval has to be taken as the initial condition for the $(n+1)$ th interval. Because the incoming units are statistically independent and identically prepared, we can treat each interaction interval as above.

A particularly important case is the limit of multiple interactions where it is reasonable to assume that the system will eventually reach a stroboscopic steady state, given that the time dependence (if any) of the system Hamiltonian $H_{S}(t)$ and of the interaction Hamiltonian $H_{X R}(t)$ with the heat reservoir is also $\tau$ periodic. We often resort to this steady-state assumption for the applications considered in this article, which reads

$$
\rho_{S}(0)=\rho_{S}(\tau)
$$

and implies $\Delta E_{S}=0$ and $\Delta S_{S}=0$. Then, the laws of thermodynamics simplify to

$$
\begin{gathered}
0=W+Q-\Delta E_{U}, \\
\Sigma_{S}=\beta\left(W-\Delta F_{U}\right) \geq I_{S: U}(\tau) \geq 0 .
\end{gathered}
$$

To justify this steady-state assumption, we assume that the reservoir is always in the same initial state at the beginning of every interaction interval $n$ so that the system and unit evolve according to the same Kraus map $\Phi_{S U}$ over each interaction interval. Physically, this means that the reservoir remains virtually unaffected by the interactions with the system and unit. This assumption is, for instance, implicit if the system-unit dynamics is described by a ME. Without this reservoir resetting assumption, justifying the existence of the steady-state regime is a much harder task. Proceeding with this assumption, it is easy to show that there also exists a Kraus map $\Phi_{S}$ for the system alone,

$\rho_{S}(n \tau+\tau)=\Phi_{S} \rho_{S}(n \tau) \equiv \operatorname{tr}_{U}\left\{\Phi_{S U} \rho_{S}(n \tau) \rho_{U}(n \tau)\right\}$.

Importantly, $\Phi_{S}$ does not depend on the interaction interval $n$ because the initial state of the unit $\rho_{U}(n \tau)$ is always the same and because all relevant Hamiltonians are assumed $\tau$ periodic. Therefore, if a unique steady state exists, it must be $\tau$ periodic. The existence of a steady state is guaranteed by Eqs. (10) and (12), and its uniqueness can be proven if we have a strict inequality [74]

$$
D(\Phi \rho \| \Phi \sigma)<D(\rho \| \sigma) \quad(\text { for } \rho \neq \sigma) .
$$

A precise mathematical condition for this strict inequality was worked out in Ref. [75] but is hard to translate physically. For instance, if additional symmetries are present, or for pure dephasing interactions (commuting with the system Hamiltonian), it is well known that there is no unique steady state [61]. Nevertheless, for most relevant scenarios, the strict inequality (54) will be satisfied. In some examples to be considered below, the existence of a unique steady state is also a well-established experimental fact (e.g., for the micromaser treated in Sec. VIB).

Finally, let us stress that even when the steady-state regime is guaranteed, solving the combined system-unit dynamics exactly is often out of reach, especially when the system and unit are complicated systems by themselves. This is why in Sec. V we go one step further and describe various limiting regimes [corresponding to special types of interaction $H_{S U}(t)$ ], where an effective ME can be derived for the system alone, with its corresponding thermodynamic interpretation.

\section{E. Discussion}

Let us summarize what we have achieved. By allowing the system to interact with units, we showed that a new term arises in the system energy and entropy balance, Eqs. (46) and (48), respectively. It describes the unit energy and entropy changes, $\Delta E_{U}$ and $\Delta S_{U}$, respectively, in addition to the traditional terms describing the energy and entropy changes in the reservoir in terms of heat. Consequently, the entropy production $\Sigma_{S}$, which measures the irreversible losses in the system dynamics, now displays a new term 
which is given by the free-energy change of the unit $\Delta F_{U}$. This term enables new transformations that would have been impossible without the units. From an operational perspective, evaluating this free energy requires preparing the units in a known state before the interaction and measuring their state after the interaction has ended. In the next section, we examine whether $\Delta E_{U}$ and $\Delta S_{U}$ can be linked to traditional thermodynamic notions of work or heat.

Since our generalized second law $\Sigma_{S} \geq 0$ provides a bound on the possibility to extract work or to convert different states into each other, it is worth mentioning that a number of different bounds have been established recently within the framework of resource theories [76-82]. These studies also explicitly show ways to saturate these bounds. While the setups they consider share some similarities with ours, the bounds obtained are, in general, different from our second law and are derived under additional restrictions imposed on the setup. For instance, within the "resource theory of thermal operations" (see Ref. [39] for an overview about different resource theories), it is assumed that the global time evolution commutes respectively with the bare Hamiltonian of the system, of the unit, and of the reservoir. This assumption is not needed within the approach presented in this section. Given that our sole restriction is to consider initially decorrelated system-unit states, the problem of finding specific protocols that saturate the bound in Eq. (49) is, in principle, equivalent to finding protocols saturating the bound (24) because the former is a consequence of the latter (see, e.g., Refs. [49,55-58] for such optimal protocols). However, these optimal protocols might correspond to highly idealized, if not unrealistic, situations. Instead of following a resource theory strategy by imposing restrictions from the start, we kept a general level of discussion in this section and will consider specific physical setups of greater experimental relevance in Sec. VI.

\section{IMPLICATIONS}

\section{A. Thermal units and ideal heat reservoir}

We consider thermal units initially prepared in an equilibrium state at an inverse temperature $\beta^{\prime}$, i.e., $\rho_{U}(0)=$ $\rho_{\beta^{\prime}}^{U}=e^{-\beta^{\prime} H_{U}} / Z_{U}$.

We say that these units behave as an ideal heat reservoir when

$$
\frac{\Delta S_{U}}{\Delta E_{U}}=\frac{1}{T^{\prime}} \quad \text { (ideal heat reservoir). }
$$

More insight is obtained by using an argument similar to the one used in Sec. II B to define an ideal heat reservoir. Using the identity

$$
T^{\prime} D\left[\rho_{U}(\tau) \| \rho_{\beta^{\prime}}^{U}\right]=\Delta E_{U}-T^{\prime} \Delta S_{U} \geq 0,
$$

we see that Eq. (55) is fulfilled when the state of the unit remains close to thermal equilibrium after the interaction, i.e., when $\rho_{U}(\tau)=\rho_{\beta^{\prime}}^{U}+\epsilon \sigma_{U}$, where $\epsilon$ is a small parameter and $\operatorname{tr}_{U}\left(\sigma_{U}\right)=0$. Indeed, we then get that $D\left[\rho_{U}(\tau) \| \rho_{\beta^{\prime}}^{U}\right]=\mathcal{O}\left(\epsilon^{2}\right)$, whereas $\Delta E_{U}$ and $\Delta S_{U}$ are, in general, nonzero and equal to first order in $\epsilon$. Since the unit energy change can be interpreted as heat, $\Delta E_{U}=-Q_{U}$, the second law (48) becomes $\Sigma_{S} \equiv \Delta S_{S}-\beta^{\prime} Q_{U}-\beta Q \geq 0$. We remark that saturating Eq. (55) away from the weak-coupling limit is, in general, nontrivial, but see Sec. VA 4 for another class of ideal heat reservoirs.

As a simple application, we operate our setup in the steady-state regime with hot thermal units $T^{\prime}>T$. Using Eq. (56) in Eq. (49), the entropy production bound implies

$W-\left(1-T / T^{\prime}\right) \Delta E_{U} \geq T D\left[\rho_{U}(\tau) \| \rho_{\beta^{\prime}}^{U}\right]+T I_{S: U}(\tau)$.

This shows that to operate as a heat engine, where work is extracted $(W<0)$, energy must be extracted from the units $\left(\Delta E_{U}<0\right)$. For thermal units constituting an ideal heat reservoir, the thermodynamic efficiency of the engine is defined as $\eta=-W / \Delta E_{U}$ and is upper bounded by the Carnot efficiency $\eta \leq 1-T / T^{\prime}$ because of Eq. (57), which reduces to $W-\left(1-T / T^{\prime}\right) \Delta E_{U} \geq 0$ in that case. However, nonideal thermal units decrease the efficiency bound as

$\eta \leq 1-\frac{T}{T^{\prime}}-T \frac{D\left[\rho_{U}(\tau) \| \rho_{\beta^{\prime}}^{U}\right]+I_{S: U}(\tau)}{-\Delta E_{U}} \leq 1-\frac{T}{T^{\prime}}$.

While realizing that a thermal stream of unit can behave as an ideal reservoir is interesting, the importance of our setup is that it allows us to treat units initially prepared out of equilibrium. One way to do it here is to consider an initial state $\rho_{\beta^{\prime}}^{U}$ with negative $\beta^{\prime}$. The efficiency of the heat engine can then formally exceed 1 without violating the second law of thermodynamics because the entropy production (47) is still non-negative. However, in this case-as the work output has to be compared with the total energy put into the system - the first law of thermodynamics tells us that a correctly defined efficiency would still be bound by one.

\section{B. Work reservoir}

To make the units act as a work source, we should engineer their state and interaction with the system in such a way that only energy is exchanged but not entropy. Thus, we define

$$
\frac{\Delta S_{U}}{\Delta E_{U}} \rightarrow 0 \quad \text { (ideal work reservoir). }
$$

For finite $\Delta E_{U}$, this implies that the change in nonequilibrium free energy is given by $\Delta F_{U}=\Delta E_{U}$, and Eq. (49) becomes, at steady state $\left(\Delta F_{S}=0\right)$, 


$$
\Sigma_{S}=\beta\left(W-\Delta E_{U}\right) \geq I_{S: U}(\tau) \geq 0
$$

This means that we can extract work from the energy initially stored in the units, but not by extracting heat from the reservoir since Eq. (60) implies that $Q<0$ because of the first law. Definition (59) can be fulfilled for many different situations depending on the precise nature of the interaction and the state and Hamiltonian of the units (we will treat particular examples in Secs. VA 3, V C, and VIB).

Let us note that for the special case of an ideal heat reservoir, which is thermal before and after the interaction (as discussed above), the form of Eqs. (59) and (60) can be obtained by choosing $T^{\prime} \rightarrow \infty$ in Eq. (55) or (56) while keeping $\Delta F_{U}$ finite. This confirms the colloquial saying that a work reservoir corresponds to an infinite-temperature heat reservoir [83].

Finally, we note that the notion of work for small systems is subtle and has been debated [6,85-92]. This originates from the desire to explain work microscopically in contrast to the standard approach where it is usually incorporated "by hand" as a time-dependent part of the system Hamiltonian. We see that the repeated interaction framework brings an interesting perspective on this issue by defining work as the part of the energy exchange that does not induce any entropy change in the units. This approach agrees with the point of view advertised in Refs. [87,88,90]. In Sec. V C, we provide an explicit model where the units effectively mimic a time-dependent system Hamiltonian and fulfill Eq. (59).

\section{Information reservoir}

We now consider the regime where the units operate as a pure information source by demanding that the exchange of energy $\Delta E_{U}$ vanishes whereas the exchange of entropy $\Delta S_{U}$ remains finite. Thus, in contrast to Eq. (59), we demand that

$$
\left(\frac{\Delta S_{U}}{\Delta E_{U}}\right)^{-1} \rightarrow 0 \quad \text { (ideal information reservoir) }
$$

Then, $\Delta F_{U}=-T \Delta S_{U}$, and the second law of thermodynamics (49) becomes at steady state $\left(\Delta F_{S}=0\right)$,

$$
\Sigma_{S}=\beta W+\Delta S_{U} \geq I_{S: U}(\tau) \geq 0 .
$$

This shows that it is possible to extract work $(W<0)$ while writing information to the units $\left(\Delta S_{U}>0\right)$ in an energy neutral fashion $\left(\Delta E_{U}=0\right)$. Note that for this interpretation, we have tacitly equated the entropy of a system with its information content in the spirit of Shannon's fundamental work [93]. Engines that are able to extract work only at the expense of information are also called information-driven engines [94].
The idea that an information reservoir represents the opposite of a work reservoir also becomes manifest by considering the case of an ideal heat reservoir in the limit $T^{\prime} \rightarrow 0$. Rearranging Eq. (56) yields

$$
\frac{\Delta E_{U}}{T^{\prime}}=\Delta S_{U}+D\left[\rho_{U}(\tau) \| \rho_{\beta^{\prime}}^{U}\right]
$$

In the limit of an ideal reservoir, the second term on the right-hand side becomes negligibly small. However, in order to keep $\Delta S_{U}$ finite while $T^{\prime} \rightarrow 0$, we automatically see that we obtain the requirement $\Delta E_{U} \rightarrow 0$. This can be achieved, for instance, by scaling the Hamiltonian of the units as $H_{U}=T^{\prime} \tilde{H}_{U}$ (note that $\tilde{H}_{U}$ is dimensionless now). The same conclusion was also reached in Ref. [95].

The notion of an "information reservoir" was introduced in a classical context by Deffner and Jarzynski in Ref. [48], where each single informational state corresponds to a set of microscopic states which are assumed to rapidly equilibrate internally. If the free-energy barriers between the different informational states are large, this enables a stable encoding of the information. Here instead, we equate each microstate of the unit with an informational state. In this respect, we do not impose any stability condition on our information, but we take all changes at the microscopic level into account. A correspondence between the two approaches can be established using a coarse-graining procedure similar to Ref. [96]. Furthermore, the thermodynamics of information reservoirs has attracted a lot of attention recently as a number of model studies show [35,36,95,97-103]. In Sec. VI A, we propose a microscopic model for the Mandal-Jarzynski engine [35], where the extracted work is shown to correspond to $W_{\text {sw }}$, Eq. (44).

An overview of the last three sections is represented in Fig. 3.

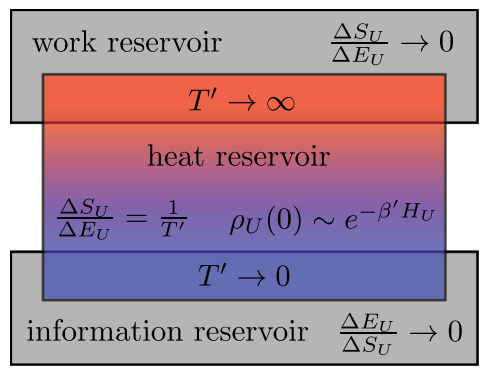

FIG. 3. Venn diagram of the thermodynamic role of the stream of units. In general, the interaction can be arbitrary, but if the initial state of the units is thermal, they can mimic an ideal heat reservoir when they fulfill the Clausius equality (55). In the limiting case where $T^{\prime} \rightarrow \infty$, we obtain a work reservoir (Sec. IV B). The converse is not true; i.e., not every work reservoir can be obtained as a limiting case of a heat reservoir. Similarly, we can obtain an information reservoir (Sec. IV C) out of a heat reservoir for $T^{\prime} \rightarrow 0$, but again the converse is not true. We note that some setups do not fit in any of the three categories. 


\section{Landauer's principle}

Landauer's principle colloquially states that logically irreversible computation-more specifically, the erasure of a bit-has a fundamental work cost. This result was first derived for a particular model by Landauer in 1961 [104]. Since then, many groups made this statement more precise by deriving it from a more general context, partly also within a repeated interaction framework, and by extending it to finite-time operations $[17,20,49,50,55,105-115]$. The modern understanding is that this principle immediately follows from the nonequilibrium version of the second law as we will show below. There is also growing experimental evidence in favor of it [116-122]. Nevertheless, this principle remains under debate [123].

Within our framework, Landauer's principle can be formulated as follows: Changing the information content (that is to say, the Shannon or von Neumann entropy) of a unit by $-\Delta S_{U}>0$ requires a work expenditure of at least

$$
\beta\left(W-\Delta E_{U}\right) \geq-\Delta S_{U}>0 .
$$

This statement immediately follows from our generalized second law (49), where $\Delta E_{S}=0$ because we focus on the steady-state regime. Note that, since $-\Delta S_{U}=S_{U}(0)-$ $S_{U}(\tau)>0$, we are indeed erasing information; i.e., we lower the Shannon or von Neumann entropy of the unit. Furthermore, we recover the standard statement $\beta W \geq$ $-\Delta S_{U}$ for $\Delta E_{U}=0$, which is automatically fulfilled if the states of the units are energetically degenerate, as is usually assumed (treatments including energetic changes can be found in Refs. [55,110] and are in agreement with our result and were also confirmed experimentally in Ref. [122]).

We emphasize that the initial product state of the system and unit, $\rho_{X}(0)=\rho_{S}(0) \rho_{U}(0)$, is essential for deriving Landauer's bound. In fact, we regard the unit (functioning as a memory in this case) as an auxiliary system to which the experimenter has free access. If the memory was initially correlated with the system, it should be treated as part of the system instead [45]. In the presence of initial correlations, it is well known that Landauer's bound does not hold $[49,124,125]$.

We end with some remarks. As pointed out in Ref. [20], erasing information is not necessarily a thermodynamically irreversible process because when reaching the equality in Eq. (64), the process becomes thermodynamically reversible (i.e., with no entropy production). The inverse operation of erasure corresponds to a randomization of the memory back to its initial state while absorbing heat from the reservoir. This can be viewed as creating information in the sense of Shannon. However, it is not a computational process, i.e., a deterministic operation on the set of logical states which cannot increase the Shannon entropy of the state during computation [126]. It is only in this sense that a logically irreversible computer can be said to produce irretrievable losses of energy. In fact, the informationdriven engines introduced in Sec. IV C can be seen as an implementation of the reverse process. The duality between work extraction and information erasure was also noticed in Refs. [35,36,48,95,98,100-103].

\section{E. Second law of thermodynamics for discrete feedback control}

Feedback control describes setups where one manipulates the dynamics of a system based on the information that one obtains by measuring it. Several groups have established that for a system undergoing feedback control in contact with a thermal reservoir at inverse temperature $\beta$, the amount of extractable work $W^{\mathrm{fb}}$ is bounded by (details about the assumptions are stated below) [56,127-138]

$$
-\beta W^{\mathrm{fb}} \leq I_{S: U}^{\mathrm{ms}},
$$

where $I_{S: U}^{\mathrm{ms}}$ is the classical mutual information [which can be obtained from Eq. (11) by replacing the von Neumann by the Shannon entropy] between the system and the memory in which the measurement result is stored after the measurement. Equation (65) is also called the second law of thermodynamics for discrete feedback control. It was confirmed experimentally in Refs. [139-141].

To be more specific, the inequality (65) holds under special conditions. For instance, the bound is known to be different for quantum systems [56], and even classically, additional requirements are imposed on the measurement which are seldom stated explicitly. Within our framework, we show that we are able to provide a very transparent and clean proof of Eq. (65).

The memory used to store the measurement of the system will be a unit in our setup. The assumption of an initially decorrelated system-memory state complies with the notion of a memory used in Sec. IV D. We assume that the Hamiltonian $H_{U}$ of the memory is completely degenerate so that the change in energy of the memory is always zero, $\Delta E_{U}=0$. The stream of memories can thus be viewed as the information reservoir introduced in Sec. IV C. Including changes in the energy of the memory poses no fundamental challenge but would just lengthen the equations below.

We now divide the interaction interval in two parts, $[0, \tau)=\left[0, t_{\mathrm{ms}}\right) \cup\left[t_{\mathrm{ms}}, \tau\right)$ with $t_{\mathrm{ms}} \in(0, \tau)$, as illustrated in Fig. 4. The measurement is performed during $\left[0, t_{\mathrm{ms}}\right)$, whereas the feedback step is performed during $\left[t_{\mathrm{ms}}, \tau\right)$.

One possibility is to treat an instantaneous measurement, $t_{\mathrm{ms}} \rightarrow 0$. In this case, the measurement consists of a deltafunction time dependence of the interaction Hamiltonian, $H_{S U}(t)=\delta(t) V_{S U}^{\mathrm{ms}}$, which generates a sudden unitary operation $U_{\mathrm{ms}}$ acting on the joint system-memory space. The state of the system and memory after such a measurement reads 


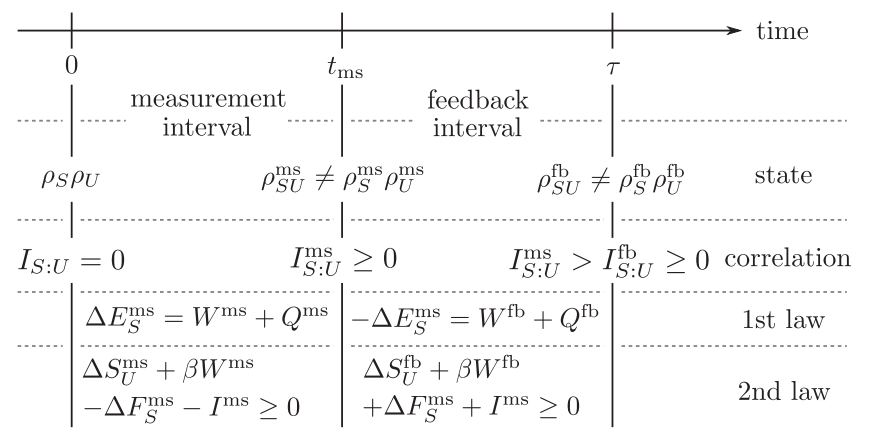

FIG. 4. Overview of the various quantities involved during the measurement and feedback step in the steady-state regime.

$$
\rho_{S U}^{\mathrm{ms}}=U_{\mathrm{ms}} \rho_{S}(0) \rho_{U} U_{\mathrm{ms}}^{\dagger},
$$

where $U_{\mathrm{ms}}=\exp \left(-i V_{S U}^{\mathrm{ms}}\right)$ and $\rho_{S U}^{\mathrm{ms}}$ will, in general, be correlated. During this short time window, the system and memory are effectively decoupled from the reservoir and the measurement acts in an entropy-preserving fashion. As a result, during $\left[0, t_{\mathrm{ms}}\right)$, the first and second laws respectively read

$$
\begin{gathered}
\Delta E_{S}^{\mathrm{ms}}=W^{\mathrm{ms}} \\
\Sigma^{\mathrm{ms}}=\Delta S_{S U}^{\mathrm{ms}}=\Delta S_{S}^{\mathrm{ms}}+\Delta S_{U}^{\mathrm{ms}}-I_{S: U}^{\mathrm{ms}}=0 .
\end{gathered}
$$

The non-negative mutual information that has been created during the measurement step, $I_{S: U}^{\mathrm{ms}}$, will constitute the resource during the feedback step.

On the other hand, if the measurement time $t_{\mathrm{ms}}$ remains finite, we are effectively implementing an "environmentally assisted measurement." If $\mathcal{L}_{\mathrm{ms}}(t)$ denotes the superoperator governing the time evolution of the system and memory in weak contact with the reservoir during $\left[0, t_{\mathrm{ms}}\right)$, then their initial state will be mapped into the generically correlated state [see Eq. (29)]

$$
\rho_{S U}^{\mathrm{ms}}=\left[\mathcal{T}_{+} \exp \int_{0}^{t_{\mathrm{ms}}} d s \mathcal{L}_{\mathrm{ms}}(s)\right] \rho_{S}(0) \rho_{U}
$$

In this case, heat exchanges with the reservoir $Q^{\mathrm{ms}}$ will occur, and the first and second laws respectively read

$$
\begin{gathered}
\Delta E_{S}^{\mathrm{ms}}=W^{\mathrm{ms}}+Q^{\mathrm{ms}}, \\
\Sigma^{\mathrm{ms}}=\Delta S_{S U}^{\mathrm{ms}}-\beta Q^{\mathrm{ms}} \\
=\Delta S_{S}^{\mathrm{ms}}+\Delta S_{U}^{\mathrm{ms}}-I_{S: U}^{\mathrm{ms}}-\beta Q^{\mathrm{ms}} \geq 0 .
\end{gathered}
$$

By combining these two laws, we find that the measurement work is bounded by

$$
-\beta W^{\mathrm{ms}} \leq-\beta \Delta F_{S}^{\mathrm{ms}}+\Delta S_{U}^{\mathrm{ms}}-I_{S: U}^{\mathrm{ms}} .
$$

A reversible implementation of the measurement, $\Sigma^{\mathrm{ms}}=0$, is possible in the limit $t_{\mathrm{ms}} \rightarrow \infty$ as examined explicitly by Bennett and others [17,94,105,106].

We remark that from the system's perspective, the measurement simply changes its state from $\rho_{S}(0)$ to $\rho_{S}^{\mathrm{ms}}=\operatorname{tr}_{U}\left(\rho_{S U}^{\mathrm{ms}}\right) \equiv \Phi_{S}^{\mathrm{ms}} \rho_{S}(0)$, where $\Phi_{S}^{\mathrm{ms}} \quad$ denotes the Kraus map of the measurement. So far, our approach is very general since it includes any kind of measurement scenario (including measurements on classical systems) compatible with an initially decorrelated system-memory state $[43,45]$.

We now turn to the feedback step. In a macroscopic setting, the observer would make a projective measurement of the memory in the computational basis $|o\rangle_{U}$. After reading the outcome $o$ of the measurement, the observer would subsequently perform a feedback step by accordingly changing the system Hamiltonian and/or the systemreservoir coupling. In the inclusive approach which we now follow, invoking a macroscopic agent is not necessary since the same resulting dynamics can be obtained by using a total Hamiltonian of the form [142]

$$
H_{\mathrm{fb}}(t)=\sum_{o} \Pi_{o} \otimes\left[H_{S}^{(o)}(t)+H_{S R}^{(o)}\right]+H_{R}
$$

Here, $\Pi_{o}=|o\rangle_{U}\langle o|$ denotes the projector onto the unit subspace corresponding to outcome $o$. The unit plays the role of a minimal description of the external agent or feedback controller. We note that the idea of describing a measured and feedback-controlled system in a larger space without having to rely on explicit measurements is not new and is at the heart of coherent or autonomous feedback in quantum mechanics [143-145]. It also works classically [146]. More details on such descriptions and on strategies to optimize work extraction can be found in Ref. [56]. We now proceed with the first and second laws of thermodynamics during the feedback step, which take the form (independently of the measurement scheme) [147]

$$
\begin{gathered}
\Delta E_{S}^{\mathrm{fb}}=W^{\mathrm{fb}}+Q^{\mathrm{fb}}, \\
\Sigma_{S}^{\mathrm{fb}}=\Delta S_{S}^{\mathrm{fb}}+\Delta S_{U}^{\mathrm{fb}}-\beta Q^{\mathrm{fb}}+I_{S: U}^{\mathrm{ms}} \geq I_{S: U}^{\mathrm{fb}} \geq 0,
\end{gathered}
$$

where $I_{S: U}^{\mathrm{ms}}$ is the system-memory mutual information at the end of the measurement interval while $I_{S: U}^{\mathrm{fb}} \equiv I_{S: U}(\tau)$ is the remaining mutual information at the end of the entire interval. We note that this latter resource is left out and will always diminish the amount of extractable work. By combining Eq. (75) with Eq. (74), we get

$$
-\beta W^{\mathrm{fb}} \leq-\beta \Delta F_{S}^{\mathrm{fb}}+\Delta S_{U}^{\mathrm{fb}}+I_{S: U}^{\mathrm{ms}} .
$$

Assuming now that we operate in the steady-state regime where $\Delta F_{S}=\Delta F_{S}^{\mathrm{ms}}+\Delta F_{S}^{\mathrm{fb}}=0$, Eq. (76) becomes 


$$
-\beta W^{\mathrm{fb}} \leq \beta \Delta F_{S}^{\mathrm{ms}}+\Delta S_{U}^{\mathrm{fb}}+I_{S: U}^{\mathrm{ms}} .
$$

This result can be regarded as the generalized second law for discrete feedback control.

To recover Eq. (65) from Eq. (77), one needs to consider a nondisturbing classical measurement in which the state of the system before and after the measurement is the same [45] and the information stored in the memory is classical. These assumptions have been used implicitly or explicitly in the classical context [127-129,131-138], whereas for quantum treatments only the information stored in the memory was treated classically $[56,130]$. Indeed, the first property of a nondisturbing measurement implies that $\Delta F_{S}^{\mathrm{ms}}=0$, while the assumption of a classical memory implies that, after the measurement, the memory is diagonal in its computational basis $|o\rangle_{U}$. Therefore, the evolution caused by the Hamiltonian (73) will leave the entropy of the memory constant, i.e., $\Delta S_{U}^{\mathrm{fb}}=0$. Hence, we recover Eq. (65), having clearly identified the necessary additional assumptions. It is worth pointing out that even classical measurements can be disturbing [45] as, in fact, any real measurement is. Then, $\Delta F_{S}^{\mathrm{ms}} \neq 0$, and the amount of extractable work changes.

We conclude this section by noting that Eq. (77) gives a bound on the extractable work during the feedback process but neglects the work invested during the measurement step (which, however, can be zero). When adding Eq. (77) to Eq. (72), we find that the total extractable work is bounded by minus the entropy change in the unit

$$
\beta W=\beta\left(W^{\mathrm{ms}}+W^{\mathrm{fb}}\right) \geq-\Delta S_{U} .
$$

This result is equivalent to Eq. (62) and shows that our feedback control scheme (implemented by a stream of memories) is equivalent to the information reservoir described in Sec. IV C. This connection between feedback control and information-driven engines was debated in Refs. [102,148-150] but is unambiguous here.

A summary of the thermodynamics of feedback control within our framework is given in Fig. 4. A model-system application will also be provided in Sec. VID.

\section{EFFECTIVE MASTER EQUATIONS}

The thermodynamic framework introduced in Sec. III is very general, and it allowed us to derive important exact identities. But in practice, it can only be used if one is able to solve the reduced dynamics of the joint system-unit complex. This is usually not an easy task. Our goal in this section will be to derive a closed reduced description for the system only, which includes its dynamics and thermodynamics. We derive effective MEs for the system which do not rely on the weak-coupling approximation, contrary to the results of Sec. II C. These MEs often have an apparent nonthermal character. For instance, they do not obey the condition (31). Establishing a consistent thermodynamics for these MEs, when solely considered as mathematical objects, can thus be challenging and often requires the ad hoc introduction of effective new quantities, which lack a solid physical interpretation [151-154]. Progress has been achieved when the MEs result from the coarse graining of a larger network of states which originally obeys a thermodynamically consistent ME, especially if the network is bipartite [96,155-159], or for particular information-driven engines [102] and "boundary-driven" MEs [160-163]. Our approach is similar in spirit since we derive effective MEs starting from the framework of repeated interactions for which we established a consistent thermodynamics.

In this section, the energy and entropy of the system at the effective level will always be given by

$$
\begin{gathered}
E_{S}(t)=\operatorname{tr}_{S}\left\{H_{S}(t) \rho_{S}(t)\right\}, \\
S_{S}(t)=-\operatorname{tr}_{S}\left\{\rho_{S}(t) \ln \rho_{S}(t)\right\} .
\end{gathered}
$$

We also allow the time interval $\tau$ between successive system-unit interactions to fluctuate according to the waiting time distribution $w(\tau)$. The duration of the interaction itself $\tau^{\prime} \leq \tau$ will be specified on a case-by-case basis. The time evolution of the system over an interval $\tau$ is given by some generic Kraus map $\Phi_{S}(\tau)$. We introduce the conditional density matrix $\rho_{S}^{(n)}(t)$, which describes the system density matrix conditioned on the fact that $n$ interactions with the units occurred so far. Then, $\rho_{S}^{(n)}(t)$ is related to $\rho_{S}^{(n-1)}(t-\tau)$ at an earlier time $\tau>0$ by

$$
\rho_{S}^{(n)}(t)=\int_{0}^{t-t_{0}} d \tau w(\tau) \Phi_{S}(\tau) \rho_{S}^{(n-1)}(t-\tau)
$$

where $t_{0}<t$ is an arbitrary initial time. The unconditional state of the system is recovered by summing over $n: \rho_{S}(t)=\sum_{n} \rho_{S}^{(n)}(t)$.

\section{A. Poisson-distributed interaction times}

\section{Setup}

In this subsection, we consider an exponential waiting time distribution $w(\tau)=\gamma e^{-\gamma \tau}$. This means that the number $N$ of units with which the system interacts during a fixed time window $T$ is Poisson distributed, i.e., $P_{N}(T)=$ $\left[(\gamma T)^{N} / N !\right] e^{-\gamma T}$. The average time between successive interactions is therefore $\int_{0}^{\infty} \tau w(\tau) d \tau=\gamma^{-1}$.

Furthermore, we assume that the system-unit interactions are very strong and short but happen very rarely. In this way, we can assure that the units have a finite influence on the evolution of the density matrix. More specifically, if the random times at which a new unit interaction occur are denoted $t_{n}(n=0,1,2, \ldots)$, the system-unit interaction Hamiltonian is a sum of delta kicks, 


$$
H_{S U}(t)=\sum_{n} \delta\left(t-t_{n}\right) V_{S U}
$$

as sketched in Fig. 5. This interaction creates an instantaneous unitary operation $U$ at times $t=t_{n}$ such that the system-unit state right after an interaction reads

$$
\rho_{S U}^{\prime}(t)=U \rho_{S}(t) \otimes \rho_{U} U^{\dagger}, \quad U=e^{-i V_{S U}} .
$$

Putting aside the brief system-unit interactions, most of the time the system will evolve in weak contact with a large thermal reservoir at inverse temperature $\beta$. Its dynamics will thus obey a ME of the form (25)

$$
d_{t} \rho_{S}(t)=\mathcal{L}_{0} \rho_{S}(t) \equiv-i\left[H_{S}(t), \rho_{S}(t)\right]+\mathcal{L}_{\beta} \rho_{S}(t),
$$

where $\mathcal{L}_{\beta}$ is the standard dissipator caused by the thermal reservoir. For notational simplicity, we keep the time dependence of superoperators implicit, $\mathcal{L}=\mathcal{L}(t)$.

Similar interaction scenarios have been considered in the past but for different purposes. See, e.g., Refs. [32,102,148,164-167].

Overall, the system evolution over an entire interval $\tau$ is given by the Kraus map

$$
\Phi_{S}(\tau) \rho_{S}=e^{\mathcal{L}_{0} \tau} \mathcal{J}_{S} \rho_{S}
$$

where

$$
\mathcal{J}_{S} \rho_{S}(t) \equiv \operatorname{tr}_{U}\left\{U \rho_{S}(t) \otimes \rho_{U} U^{\dagger}\right\}
$$

Thus, Eq. (85) describes the short kick felt by the system due to the interaction with the unit $\left(\mathcal{J}_{S}\right)$ followed by the dissipative evolution of the system in contact with the reservoir $\left(e^{\mathcal{L}_{0} \tau}\right)$. If $\mathcal{L}_{0}$ is time dependent, we have to replace $e^{\mathcal{L}_{0} \tau}$ by the corresponding time-ordered generator; see Eq. (29). For later convenience, we also introduce the superoperator describing the effect of a system-unit interaction on the unit

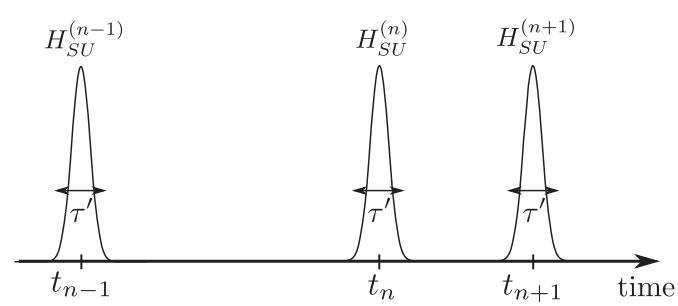

FIG. 5. Sketch of the Poisson-distributed regime: The system evolves freely most of the time but, once in a while, undergoes a short $\left(\tau^{\prime} \rightarrow 0\right)$ and strong $\left(H_{S U} \sim \tau^{\prime-1}\right)$ interaction with a unit where $\tau^{\prime}$ denotes the duration of the interaction as in Sec. III. The duration $\tau$ between successive system-unit interactions fluctuates according to an exponential time distribution with average duration $\gamma^{-1}$.

$$
\mathcal{J}_{U} \rho_{U} \equiv \operatorname{tr}_{S}\left\{U \rho_{S}(t) \otimes \rho_{U} U^{\dagger}\right\} .
$$

Note that $\mathcal{J}_{U}=\mathcal{J}_{U}(t)$ might be time dependent if $\rho_{S}(t)$ has not yet reached its steady state, but $\mathcal{J}_{S}$ is not.

Using Eq. (85) in Eq. (81) and substituting $\tau=t-t^{\prime}$ to make the dependence on the actual time $t$ explicit, we obtain

$$
\rho_{S}^{(n)}(t)=\int_{t_{0}}^{t} d t^{\prime} \gamma e^{-\gamma\left(t-t^{\prime}\right)} e^{\mathcal{L}_{0}\left(t-t^{\prime}\right)} \mathcal{J}_{S} \rho_{S}^{(n-1)}\left(t^{\prime}\right) .
$$

By taking the time derivative, we find

$d_{t} \rho_{S}^{(n)}(t)=-\gamma \rho_{S}^{(n)}(t)+\mathcal{L}_{0} \rho_{S}^{(n)}(t)+\gamma \mathcal{J}_{S} \rho_{S}^{(n-1)}(t)$,

and by summing over $n$, we finally obtain the effective ME ruling the averaged time evolution of the system,

$$
d_{t} \rho_{S}(t)=\mathcal{L}_{0} \rho_{S}(t)+\gamma\left(\mathcal{J}_{S}-1\right) \rho_{S}(t) .
$$

We can make the new part $\gamma\left(\mathcal{J}_{S}-1\right)$ of the ME (90) more explicit by writing the initial state of the unit as

$$
\rho_{U}=\sum_{k} p_{k}|k\rangle_{U}\langle k|,
$$

where $\left\{|k\rangle_{U}\right\}$ is an arbitrary set of eigenstates of $\rho_{U}$ (not necessarily energy eigenstates of $H_{U}$ ). Then, we see that

$$
\mathcal{J}_{S} \rho_{S}(t)=\sum_{k, l} A_{k l} \rho_{S}(t) A_{k l}^{\dagger}
$$

has the form of a Kraus map (4) where the system operators defined as $A_{k l} \equiv \sqrt{p_{k}}\langle l|U| k\rangle_{U}$ fulfill the completeness relation $\sum_{k, l} A_{k l}^{\dagger} A_{k l}=\mathbf{1}_{S}$. Therefore, we can write

$$
\mathcal{L}_{\text {new }} \rho_{S}(t) \equiv \gamma\left(\mathcal{J}_{S}-1\right) \rho_{S}(t)=\gamma \sum_{k, l} \mathcal{D}\left[A_{k l}\right] \rho_{S}(t),
$$

where $\mathcal{D}[A] \rho \equiv A \rho A^{\dagger}-\frac{1}{2}\left\{A^{\dagger} A, \rho\right\}$, thus explicitly showing that $\mathcal{L}_{\text {new }}$ is of Lindblad form $[61,69,70]$. By choosing $\rho_{U}$ and $V_{S U}$ appropriately, we can create arbitrary $A_{k l}$ 's as long as they fulfill the completeness relation. Note that the class of generators $\mathcal{L}_{\text {new }}$ created this way is not equivalent to the class of thermal generators (26). In general, relation (30) or (31) will not be fulfilled for $\mathcal{L}_{\text {new }}$. Furthermore, we remark that including multiple independent streams of units can be easily done within this scenario because the probability of a simultaneous interaction with more than one unit is negligible.

In summary, the new ME (90) can be written as

$d_{t} \rho_{S}(t)=-i\left[H_{S}(t), \rho_{S}(t)\right]+\mathcal{L}_{\beta} \rho_{S}(t)+\mathcal{L}_{\text {new }} \rho_{S}(t)$.

\section{Thermodynamics}

We now turn to the thermodynamic description corresponding to the setup above. 
We start by considering energy and entropy changes in the units. During a short time interval $d t$, the probability that a unit interacts (resp. does not interact) is given by $\gamma d t \ll 1$ (resp. $1-\gamma d t$ ). In the former case, the unit state changes from $\rho_{U}$ to $\mathcal{J}_{U} \rho_{U}$, while it remains in $\rho_{U}$ in the latter case. Since an energy and entropy change in the unit only occurs when an interaction takes place, the rates of unit energy and entropy change are given by

$$
\begin{aligned}
d_{t} E_{U}(t)= & \gamma\left(\operatorname{tr}_{U}\left\{H_{U} \mathcal{J}_{U} \rho_{U}\right\}-\operatorname{tr}_{U}\left\{H_{U} \rho_{U}\right\}\right), \\
d_{t} S_{U}(t)= & \gamma\left(-\operatorname{tr}_{U}\left\{\left(\mathcal{J}_{U} \rho_{U}\right) \ln \left(\mathcal{J}_{U} \rho_{U}\right)\right\}\right. \\
& \left.+\operatorname{tr}_{U}\left\{\rho_{U} \ln \rho_{U}\right\}\right) .
\end{aligned}
$$

We now turn to the rate of work injected in the joint system unit, which we again split into two parts:

$$
\dot{W}=\dot{W}_{S}+\dot{W}_{S U},
$$

where

$$
\begin{gathered}
\dot{W}_{S}=\operatorname{tr}_{S}\left\{\rho_{S}(t) d_{t} H_{S}(t)\right\}, \\
\dot{W}_{S U}=\gamma \operatorname{tr}_{S U}\left\{\left[H_{S}(t)+H_{U}\right]\left[U \rho_{S}(t) \rho_{U} U^{\dagger}-\rho_{S}(t) \rho_{U}\right]\right\} \\
=\gamma \operatorname{tr}_{S}\left\{H_{S}(t)\left(\mathcal{J}_{S}-1\right) \rho_{S}(t)\right\} \\
+\gamma \operatorname{tr}_{U}\left\{H_{U}\left(\mathcal{J}_{U}-1\right) \rho_{U}\right\} .
\end{gathered}
$$

The first part is the work due to the time dependence in the system Hamiltonian $H_{S}(t)$, while the second part is due to the system-unit interaction when it occurs. Since this latter gives rise to a unitary dynamics in the system-unit space which produces no heat, it is given by the energy change in the system and unit due to the system-unit interaction.

The overall change in the energy of the system is naturally given by

$$
d_{t} E_{S}(t)=d_{t} \operatorname{tr}_{S}\left\{H_{S}(t) \rho_{S}(t)\right\},
$$

and the heat entering the system from the reservoir is

$$
\begin{aligned}
\dot{Q}(t) & =\operatorname{tr}_{S}\left\{H_{S}(t) \mathcal{L}_{\beta} \rho_{S}(t)\right\} \\
& =-\beta^{-1} \operatorname{tr}_{S}\left\{\left[\mathcal{L}_{\beta} \rho_{S}(t)\right] \ln \rho_{\beta}^{S}(t)\right\} .
\end{aligned}
$$

Noting that $\dot{W}_{S U}(t)-d_{t} E_{U}(t)=\gamma \operatorname{tr}_{S}\left\{H_{S}(t) \mathcal{L}_{\text {new }} \rho_{S}(t)\right\}$, we obtain the first law of thermodynamics,

$$
d_{t} E_{S}(t)=\dot{Q}(t)+\dot{W}(t)-d_{t} E_{U}(t),
$$

which constitutes the differential version of the general result Eq. (46).

We now proceed to show that the differential version of the generalized second law (48),

$$
\dot{\Sigma}_{S}(t)=d_{t} S_{S}(t)+d_{t} S_{U}(t)-\beta \dot{Q} \geq 0,
$$

also holds and that its non-negativity is ensured. Using the fact that $d_{t} S_{S}(t)=-\operatorname{tr}\left\{\left[d_{t} \rho_{S}(t)\right] \ln \rho_{S}(t)\right\}$ together with (90), we find that

$$
\begin{aligned}
d_{t} S_{S}(t)= & -\operatorname{tr}_{S}\left\{\left[\mathcal{L}_{\beta} \rho_{S}(t)\right] \ln \rho_{S}(t)\right\} \\
& -\gamma \operatorname{tr}_{S}\left\{\left[\left(\mathcal{J}_{S}-1\right) \rho_{S}(t)\right] \ln \rho_{S}(t)\right\} .
\end{aligned}
$$

Combining this with Eq. (101), we can rewrite Eq. (103) as

$$
\begin{aligned}
\dot{\Sigma}_{S}(t)= & -\operatorname{tr}_{S}\left\{\mathcal{L}_{\beta} \rho_{S}(t)\left[\ln \rho_{S}(t)-\ln \rho_{\beta}^{S}(t)\right]\right\} \\
& -\gamma \operatorname{tr}_{S}\left\{\left[\left(\mathcal{J}_{S}-1\right) \rho_{S}(t)\right] \ln \rho_{S}(t)\right\}+d_{t} S_{U}(t) \\
\geq & -\gamma \operatorname{tr}_{S}\left\{\left[\left(\mathcal{J}_{S}-1\right) \rho_{S}(t)\right] \ln \rho_{S}(t)\right\}+d_{t} S_{U}(t),
\end{aligned}
$$

where we used Spohn's inequality (36) at the end. Using Eq. (96), the remaining part can be expressed as

$$
\begin{aligned}
\frac{\dot{\Sigma}_{S}(t)}{\gamma} \geq & -\operatorname{tr}_{S}\left\{\left[\mathcal{J}_{S} \rho_{S}(t)\right] \ln \left[\mathcal{J}_{S} \rho_{S}(t)\right]\right\}+D\left[\mathcal{J}_{S} \rho_{S}(t) \| \rho_{S}(t)\right] \\
& -\operatorname{tr}_{S}\left\{\rho_{S}(t) \ln \rho_{S}(t)\right\}-\operatorname{tr}_{U}\left\{\left(\mathcal{J}_{U} \rho_{U}\right) \ln \left(\mathcal{J}_{U} \rho_{U}\right)\right\} \\
& +\operatorname{tr}_{U}\left\{\rho_{U} \ln \rho_{U}\right\} .
\end{aligned}
$$

Now using the fact that entropy does not change under unitary transformation so that

$$
\begin{aligned}
S\left[\rho_{S}(t)\right]+S\left[\rho_{U}\right]= & S\left[\rho_{S}(t) \otimes \rho_{U}\right] \\
= & S\left[U \rho_{S}(t) \otimes \rho_{U} U^{\dagger}\right] \\
= & S\left[\mathcal{J}_{S} \rho_{S}(t)\right]+S\left[\mathcal{J}_{U} \rho_{U}\right] \\
& -I_{S: U}\left[U \rho_{S}(t) \rho_{U} U^{\dagger}\right]
\end{aligned}
$$

we can prove the non-negativity of $\dot{\Sigma}_{S}(t)$ since

$\frac{\dot{\Sigma}_{S}(t)}{\gamma} \geq D\left[\mathcal{J}_{S} \rho_{S}(t) \| \rho_{S}(t)\right]+I_{S: U}\left[U \rho_{S}(t) \rho_{U} U^{\dagger}\right] \geq 0$.

The present analysis underlines that our generalized thermodynamic framework of repeated interactions can be carried over to the limiting situation considered in this section of very short and Poisson-distributed system-unit interactions. The resulting description is closed in terms of the system density matrix obeying the dynamics (94), and the non-negativity of the differential form of the second law (103) is a stronger result than the original integrated one (47).

A crucial point to emphasize is that knowing the physical mechanism underlying a ME dynamics such as Eq. (94) is essential to establish its correct thermodynamics. Indeed, without the additional information about the units at hand, the thermodynamic analysis would be very 
different. Presupposing that we were able to disentangle the two dissipative mechanisms caused by $\mathcal{L}_{\beta}$ and $\mathcal{L}_{\text {new }}$, we would need to define an effective heat flow $\dot{Q}_{\text {eff }}(t) \equiv$ $\operatorname{tr}\left\{H_{S} \mathcal{L}_{\text {new }} \rho_{S}(t)\right\}$ to explain the discrepancy in the first law. From our inclusive approach above, however, we know that $\dot{Q}_{\text {eff }}(t)=\dot{W}_{S U}(t)-d_{t} E_{U}(t)$. The effect on the second law would be even more drastic. Using Spohn's inequality (36), we know that the quantity

$$
\begin{aligned}
\dot{\Sigma}_{S}^{\text {eff }} \equiv- & \operatorname{tr}\left\{\left[\mathcal{L}_{0} \rho_{S}(t)\right]\left[\ln \rho_{S}(t)-\ln \rho_{\beta}^{S}(t)\right]\right\} \\
& -\operatorname{tr}\left\{\left[\mathcal{L}_{\text {new }} \rho_{S}(t)\right]\left[\ln \rho_{S}(t)-\ln \bar{\rho}_{\text {new }}\right]\right\}
\end{aligned}
$$

would always be non-negative as a sum of two nonnegative terms. Here, $\bar{\rho}_{\text {new }}$ denotes the steady state of $\mathcal{L}_{\text {new }}$, i.e., $\mathcal{L}_{\text {new }} \bar{\rho}_{\text {new }}=0$. In fact, if $\mathcal{L}_{\text {new }}$ corresponds to the dissipator caused by a standard thermal reservoir, Eq. (108) would correspond to the standard entropy production. However, this is not the case; thus, $\dot{\Sigma}_{S}^{\text {eff }}$ is not only numerically different from $\dot{\Sigma}_{S}$, but it also lacks any a priori thermodynamic interpretation.

\section{Heat, work, and information-dominated interactions}

Following the line of Secs. IV A-IV C, we now consider specific scenarios that have a clear thermodynamic interpretation.

We start by studying the case of initially thermal units at inverse temperature $\beta^{\prime}$ as in Sec. IVA. Then, using Eqs. (95) and (96), we deduce, in accordance with Eq. (56), that

$$
d_{t} S_{U}(t)=\beta^{\prime} d_{t} E_{U}(t)-\gamma D\left(\mathcal{J}_{U} \rho_{U} \| \rho_{U}\right) .
$$

Thus, in contrast to a weakly coupled macroscopic reservoir, the units, in general, do not mimic an ideal heat reservoir unless additional assumptions are used, as we will see in Sec. VA 4.

The difference between the effective ME (90) and the weak-coupling $\mathrm{ME}$ from Sec. IIC also becomes apparent by noting that the generator $\mathcal{L}_{\text {new }}$ is not of the form of $\mathcal{L}_{\beta}$ in Eq. (26). Thus, initially thermal units will typically not imply that $\mathcal{L}_{\text {new }} \rho_{\beta^{\prime}}^{S}(t)=0$. One very specific way to enforce it is to assume that the units and the system have identical Hamiltonians, $H_{S}=\sum_{k} E_{k}\left|E_{k}\right\rangle_{S}\left\langle E_{k}\right|$ and $H_{U}=\sum_{k} E_{k}\left|E_{k}\right\rangle_{U}\left\langle E_{k}\right|$, and that their delta kick interaction gives rise to a unitary evolution of the form $U=\sum_{k, l}\left|E_{k}\right\rangle_{S}\left\langle E_{l}|\otimes| E_{l}\right\rangle_{U}\left\langle E_{k}\right|$, which swaps energy between the system and the unit.

For the work reservoir, we require that $d_{t} S_{U}=0$ while $d_{t} E_{U} \neq 0$. For initially thermal units, this can again be ensured by choosing $\beta^{\prime} \rightarrow 0$ as in Sec. IV B. But, in general, to ensure that the entropy of the unit remains constant while its energy can change, the effective unit dynamics should be given by a unitary operator $U_{U}$,
$\mathcal{J}_{U} \rho_{U}=U_{U} \rho_{U} U_{U}^{\dagger}$. Besides the trivial choice $U=U_{S} \otimes U_{U}$, finding such conditions might not be easy.

In turn, the limit of an information reservoir where $d_{t} E_{U}=0$ and $d_{t} S_{U}(t) \neq 0$ can be easily achieved for any interaction by considering a fully degenerate unit Hamiltonian,

$$
H_{U} \sim \mathbf{1}_{U} \quad \text { (ideal information reservoir), }
$$

naturally always implying $d_{t} E_{U}=0$.

A last important class of interactions are those generated by the unitary operator

$$
U=\sum_{k} U_{S}^{(k)} \otimes|k\rangle_{U}\langle k|,
$$

where $U_{S}^{(k)}$ is an arbitrary unitary operator in the system Hilbert space whereas $|k\rangle_{U}$ denotes the eigenvectors of $\rho_{U}$, Eq. (91). One easily verifies that the unit state does not change during the interaction, $\rho_{U}=\mathcal{J}_{U} \rho_{U}$, and hence its energy and entropy also stay constant, $d_{t} E_{U}=0, d_{t} S_{U}=0$. In this case, the units are neither a work nor an information reservoir. Instead, the system state changes according to

$$
\mathcal{J}_{S} \rho_{S}=\sum_{k} p_{k} U_{S}^{(k)} \rho_{S}\left(U_{S}^{(k)}\right)^{\dagger},
$$

where $p_{k}=\left\langle k\left|\rho_{U}\right| k\right\rangle$. This interaction will therefore, in general, inject energy as well as entropy into the system. Using Eq. (106), we see that the change in the system entropy caused by such a system-unit interaction is given by the mutual information established between the system and the unit after the interaction,

$$
S\left[\mathcal{J}_{S} \rho_{S}(t)\right]-S\left[\rho_{S}(t)\right]=I_{S: U}\left[U \rho_{S}(t) \rho_{U} U^{\dagger}\right] .
$$

Thus, the interaction (111) can be seen as a measurement of the unit by the system. Indeed, depending on the unit state $|k\rangle_{U}$, the system will, in general, change its state to $U_{S}^{(k)} \rho_{S}\left(U_{S}^{(k)}\right)^{\dagger}$. Vice versa, by exchanging the labels $U$ and $S$ above, we can also implement a measurement of the system by the units. This will be used in Sec. VID.

\section{Ensemble of units and ideal heat reservoir}

In Sec. VA 2, we considered the energy and entropy changes of those units which interacted with the system. An interesting alternative approach consists in evaluating energy and entropy changes with respect to a statistical ensemble composed of both the units that did and those that did not interact. One physically relevant scenario for this is the case where units are frequently sent to the system, but only a small Poisson-distributed fraction of them interact whereas the rest remain unchanged. 
Mathematically, let us assume that every time step $d t$, a unit passes the system with certainty but only interacts with it with probability $\gamma d t \ll 1$, which is assumed to be infinitesimal such that the average evolution of the system is still differentiable and coincides with Eq. (94). If we do not record the precise interaction times, each outgoing unit $\rho_{U}^{\prime}$ must be described by the state

$$
\rho_{U}^{\prime}=(1-\gamma d t) \rho_{U}+\gamma d t \mathcal{J}_{U} \rho_{U}
$$

where $\rho_{U}$ describes the initial state as usual. The change in unit entropy per time step $d t$ then becomes

$$
\begin{aligned}
d_{t} \bar{S}_{U}(t) & \equiv \lim _{d t \rightarrow 0} \frac{S\left(\rho_{U}^{\prime}\right)-S\left(\rho_{U}\right)}{d t} \\
& =-\gamma \operatorname{tr}\left\{\left[\left(\mathcal{J}_{U}-1\right) \rho_{U}\right] \ln \rho_{U}\right\} .
\end{aligned}
$$

Here, we used a bar to distinguish this definition from the previous case, Eq. (104), in which every unit passing the system also interacts with the system. The difference between both is exactly

$$
d_{t} \bar{S}_{U}(t)-d_{t} S_{U}(t)=\gamma D\left(\mathcal{J}_{U} \rho_{U} \| \rho_{U}\right) \geq 0,
$$

which can be interpreted as the entropy increase caused by mixing the units that interacted with the system with those that did not. In contrast, since the energy $E_{U}(t)$ of the units is a linear functional of $\rho_{U}(t)$, we easily deduce that $d_{t} \bar{E}_{U}(t)=d_{t} E_{U}(t)$. In other words, while the entropy balance differs between the two approaches, the energy balance remains the same. Since heat is also unaffected, the difference in entropy production between the two approaches reads

$$
\dot{\bar{\Sigma}}_{S}(t)=\dot{\Sigma}_{S}+\gamma D\left(\mathcal{J}_{U} \rho_{U} \| \rho_{U}\right) \geq 0
$$

An important implication of the present approach is that when considering units that are initially thermal $\rho_{U}=\rho_{\beta^{\prime}}^{U}$, we find that

$$
d_{t} \bar{S}_{U}(t)=\beta^{\prime} d_{t} \bar{E}_{U}(t),
$$

in contrast to Eq. (109). Thus, the notion of an ideal heat reservoir requires one to not only focus on those units that interacted but to consider the statistical mixture of units that did and did not interact. This picture is also supported by an alternative approach.

Consider a reservoir made of an initial population of $N_{0} \gg 1$ identical and independent units. Let us assume that the particle content of the reservoir decays exponentially, $N_{t}=N_{0} e^{-\gamma t}$, and produces the sequence of units that all eventually interact with the system. In contrast to the case in Sec. VA 2, after they interact, we send the units back to the reservoir and let them mix with the remaining $N_{t}$ fresh units. The mixed state of this reservoir can be described as

$$
\tilde{\rho}_{U}(t)=\frac{N_{0}-N_{t}}{N_{0}} \mathcal{J}_{U} \rho_{U}+\frac{N_{t}}{N_{0}} \rho_{U}
$$

where we assumed that $\mathcal{J}_{U}$ is time independent for simplicity. For such a process, we have $d_{t} N_{t}=-\gamma N_{t}$, and thus

$$
d_{t} \tilde{\rho}_{U}(t)=\gamma \frac{N_{t}}{N_{0}}\left(\mathcal{J}_{U}-1\right) \rho_{U}
$$

Considering times over which $N_{t} \approx N_{0}$, to remain consistent with the assumption that only fresh units interact with the system, it is possible to recover the same mathematical results as above. Thus, physically separating the units from the system and dividing them into an incoming and outgoing stream is not essential in this picture. One could equally well consider a gas of noninteracting units surrounding the system and interacting with it at Poisson random times.

\section{B. Regular and frequent interaction intervals}

\section{Setup}

There is another class of the repeated interaction framework that has often been considered to derive MEs [160-163,166,168,169]. Its thermodynamics has been considered as well [160-163]. In this scenario, the duration between two consecutive system-unit interactions $\tau$ is taken to be constant, as in Sec. III, and equal to the duration of the system-unit interaction $\tau^{\prime}$. Furthermore, the duration is short, and $\tau^{\prime}=\tau \equiv \delta t$ is used as a small expansion parameter, where the interaction is assumed to be of the form

$$
H_{S U}(t)=\sum_{n} \Theta(t-n \delta t) \Theta(n \delta t+\delta t-t) \frac{\tilde{V}}{\sqrt{\delta t}} .
$$

This Hamiltonian models very short but permanent and strong interactions. The fact that every unit is replaced after a time $\delta t$ implies Markovianity. Furthermore, for a clean derivation of the ME, in this setting one has to assume no coupling to a thermal reservoir. Therefore, the system and unit evolve unitarily over one period via the operator

$$
U(\delta t)=\exp \left\{-i \delta t\left[H_{S}(t)+H_{U}+\tilde{V} / \sqrt{\delta t}\right]\right\},
$$

where it is further assumed that $\delta t$ is much smaller than the rate of change of $H_{S}(t)$. Finally, one needs to expand the evolution of $\rho_{S}(t)$ up to first order in $\delta t$ under the assumption that $\operatorname{tr}\left\{\tilde{V} \rho_{U}\right\}=0$.

Instead of following such a derivation, which is presented elsewhere [160-163,166,168,169], we follow an alternative route by considering the setup of Sec. VA in the limit of an infinitely fast Poisson process. This procedure yields identical mathematical results. More specifically, we 
consider the limit where the Poisson rate scales as $\gamma=\epsilon^{-1}$ while at the same time assuming that the unitary interaction (83) scales as $U=\exp (-i \sqrt{\epsilon} \tilde{V})$, i.e., $V_{S U}=\sqrt{\epsilon} \tilde{V}$ in Eq. (82). Furthermore, we explicitly neglect the reservoir; i.e., we set $\mathcal{L}_{\beta}=0$ in the results of Sec. VA. The Kraus map (85) then reads $\Phi_{S}(\tau) \rho_{S}=e^{-i H_{S}(t) \tau}\left[\mathcal{J}_{S} \rho_{S}\right] e^{i H_{S}(t) \tau}$. Our goal now is to derive an effective ME in the limit $\epsilon \rightarrow 0$.

We start with Eq. (86) by expanding $U \rho_{S}(t) \rho_{U} U^{\dagger}$ in powers of $\sqrt{\epsilon}$. This yields

$$
\begin{aligned}
\mathcal{J}_{S} \rho_{S}(t)= & \rho_{S}(t)-i \sqrt{\epsilon} \operatorname{tr}_{U}\left\{\left[\tilde{V}, \rho_{S}(t) \rho_{U}\right]\right\} \\
& -\frac{\epsilon}{2} \operatorname{tr}_{U}\left\{\left[\tilde{V},\left[\tilde{V}, \rho_{S}(t) \rho_{U}\right]\right]\right\}+\ldots
\end{aligned}
$$

In order to derive a meaningful differential equation, we now also have to demand that the interaction $\tilde{V}$ or the initial unit state $\rho_{U}$ is chosen such that $\operatorname{tr}_{U}\left\{\tilde{V} \rho_{U}\right\}=0$, which removes the term proportional to $\sqrt{\epsilon}$. Then, we consider Eq. (93), which becomes

$$
\begin{aligned}
\mathcal{L}_{\text {new }} \rho_{S}(t) & =\frac{1}{\epsilon}\left(\rho_{S}(t)-\frac{\epsilon}{2} \operatorname{tr}_{U}\left\{\left[\tilde{V},\left[\tilde{V}, \rho_{S}(t) \rho_{U}\right]\right]\right\}+\cdots-\rho_{S}(t)\right) \\
& =\frac{1}{2} \operatorname{tr}_{U}\left\{\left[\tilde{V},\left[\tilde{V}, \rho_{S}(t) \rho_{U}\right]\right]\right\}+\mathcal{O}(\sqrt{\epsilon}) .
\end{aligned}
$$

We can make this effective ME more explicit by writing $\tilde{V}=\sum_{k} A_{k} \otimes B_{k}$, where $A_{k}$ and $B_{k}$ are arbitrary operators in the system and unit space such that $\tilde{V}$ is Hermitian. Then, after taking into account the influence of the possibly timedependent system Hamiltonian, from Eq. (94) we get, with $\mathcal{L}_{\beta}=0$, our final ME:

$$
\begin{aligned}
d_{t} \rho_{S}(t)= & -i\left[H_{S}(t), \rho_{S}(t)\right] \\
& +\sum_{k, l}\left\langle B_{l} B_{k}\right\rangle_{U}\left(A_{k} \rho_{S}(t) A_{l}-\frac{1}{2}\left\{A_{l} A_{k}, \rho_{S}(t)\right\}\right),
\end{aligned}
$$

where we defined $\left\langle B_{l} B_{k}\right\rangle_{U} \equiv \operatorname{tr}_{U}\left\{B_{l} B_{k} \rho_{U}\right\}$. This result agrees with Refs. [160-162,166,168] and will be further used in Sec. VIC. Treating multiple streams of units can also be easily done within this setup. We finally note that this ME is very similar (but not identical) to the singular coupling ME [61].

\section{Thermodynamics}

A thermodynamic analysis of such "boundary-driven MEs" (125) was given in Ref. [162] for the case of initially thermal units $\rho_{U}=\rho_{\beta}^{U}$. We now approach this problem from our perspective, demonstrating that the thermodynamic framework in Ref. [162] is consistent but overestimates the entropy production.
For $\operatorname{tr}_{U}\left\{\tilde{V} \rho_{U}\right\}=0$, it follows immediately that (in the following, we consider only the leading-order terms)

$$
\begin{aligned}
\mathcal{J}_{S} \rho_{S}(t)= & \rho_{S}(t)-\frac{\epsilon}{2} \operatorname{tr}_{U}\left\{\left[\tilde{V},\left[\tilde{V}, \rho_{S}(t) \rho_{U}\right]\right]\right\}, \\
\mathcal{J}_{U} \rho_{U}= & \rho_{U}(t)-i \sqrt{\epsilon} \operatorname{tr}_{S}\left\{\left[\tilde{V}, \rho_{S}(t) \rho_{U}\right]\right\} \\
& -\frac{\epsilon}{2} \operatorname{tr}_{S}\left\{\left[\tilde{V},\left[\tilde{V}, \rho_{S}(t) \rho_{U}\right]\right]\right\} .
\end{aligned}
$$

Thus, it is clear that all thermodynamic quantities defined in Sec. VA for the system [i.e., $d_{t} E_{S}(t)$ and $\left.d_{t} S_{S}(t)\right]$ are well behaved (i.e., do not diverge for $\epsilon \rightarrow 0$ ), but for the unit, this is less clear.

We start by looking at unit-related quantities in the first law. For instance, for Eq. (95) [the same terms appear in $\dot{W}_{S U}(t)$, too],

$$
\begin{aligned}
d_{t} E_{U}(t)= & \gamma \operatorname{tr}_{U}\left\{H_{U}\left(\mathcal{J}_{U}-1\right) \rho_{U}\right\} \\
= & -i \frac{1}{\sqrt{\epsilon}} \operatorname{tr}_{S U}\left\{H_{U}\left[\tilde{V}, \rho_{S}(t) \rho_{U}\right]\right\} \\
& -\frac{1}{2} \operatorname{tr}_{S U}\left\{H_{U}\left[\tilde{V},\left[\tilde{V}, \rho_{S}(t) \rho_{U}\right]\right]\right\},
\end{aligned}
$$

where we replaced $\gamma=\epsilon^{-1}$ and used Eq. (127). Thus, the first term will diverge as $\epsilon \rightarrow 0$ unless $\left[H_{U}, \tilde{V}\right]=0$ or $\left[H_{U}, \rho_{U}\right]=0$. Note, however, that the divergences cancel out if we consider the first law (102) for the system. Furthermore, within the framework of Ref. [162], we indeed have $\left[H_{U}, \rho_{U}\right]=0$ since $\rho_{U}=\rho_{\beta}^{U}$; hence,

$$
d_{t} E_{U}(t)=-\frac{1}{2} \operatorname{tr}_{S U}\left\{H_{U}\left[\tilde{V},\left[\tilde{V}, \rho_{S}(t) \rho_{U}\right]\right]\right\},
$$

$\dot{W}_{S U}(t)=-\frac{1}{2} \operatorname{tr}_{S U}\left\{\left(H_{S}+H_{U}\right)\left[\tilde{V},\left[\tilde{V}, \rho_{S}(t) \rho_{U}\right]\right]\right\}$,

demonstrating that $d_{t} E_{U}(t)$ and $\dot{W}_{S U}(t)$ remain well behaved. Furthermore, we can identify $\dot{W}_{S U}(t)=\dot{W}_{\text {sw }}(t)$ by using definition (44) and some straightforward algebraic manipulations.

Turning to the entropy change, we see from our result (109) that the only term that could cause a divergence is $\epsilon^{-1} D\left(\mathcal{J}_{U} \rho_{U} \| \rho_{U}\right)$. However, with the use of footnote [52] and Eq. (127), we find that $D\left(\mathcal{J}_{U} \rho_{U} \| \rho_{U}\right)=\mathcal{O}(\epsilon)$; thus, the entropy change of the units is also well behaved.

In total, the first and second laws derived in Sec. VA become, for this setup,

$$
d_{t} E_{S}(t)=\dot{W}_{S}(t)+\dot{W}_{\mathrm{sw}}(t)+d_{t} E_{U}(t),
$$

$\dot{\Sigma}_{S}(t)=d_{t} S_{S}(t)+\beta^{\prime} d_{t} E_{U}(t)-\gamma D\left(\mathcal{J}_{U} \rho_{U} \| \rho_{U}\right) \geq 0$. 
To compare our results with Ref. [162], we set $\dot{W}_{S}(t)=0$. In this reference, the change in the unit energy is identified with heat, $\dot{Q}_{U}(t) \equiv d_{t} E_{U}(t)$. The first law is the same as ours, but the second law reads $d_{t} S_{S}(t)+\beta^{\prime} \dot{Q}_{U}(t) \geq 0$. Interestingly, this is the result obtained in Sec. VA 4, when the ensemble considered is not only that of the units that interacted but the entire set of units. As we have seen, it overestimates our entropy production by a mixing term $\gamma D\left(\mathcal{J}_{U} \rho_{U} \| \rho_{U}\right)$.

Finally, this example also illustrates that-although the dynamics in the joint space of the system and all units is unitary (and thus reversible) - the dynamics of the system is irreversible precisely because we impose a unidirectional movement of the units. If we time reverse the global evolution, we would recover the initial system state. This can also be seen in the system-specific entropy production, which can be rewritten as [see also Eq. (9)] $\dot{\Sigma}_{S}(t)=d_{t} I_{S: U}(t)$; i.e., for the entropy production rate of system and unit [compare with Eq. (47)], we have $\dot{\Sigma}=0$.

\section{Mimicking time-dependent Hamiltonians}

\section{Setup}

In the last part of this section, we show that the stream of units can be engineered in a way that will effectively generate a time-dependent system Hamiltonian of the form

$$
H_{S}(t)=H_{0}+f(t) A,
$$

where $f(t)$ is an arbitrary real-valued and differentiable function and $A$ an arbitrary Hermitian system operator. We will further show that this stream of units acts as a work source, thereby providing an alternative justification for treating time-dependent Hamiltonians as work sources as done in standard quantum thermodynamics $[1,3,60]$. We note that research in the direction of obtaining a timedependent Hamiltonian out of a time-independent one has been carried out for different settings in Refs. [89,170,171].

The idea is that an arbitrary drive $f(t)$ can be effectively generated by a stream of units with system-unit interactions of the form $A \otimes F$, where so far $F$ is an unspecified Hermitian unit operator. As in the previous subsection, we consider short and repeated interactions: $\tau^{\prime}=\tau \equiv \delta t$. We also consider no reservoir at the moment. However, since $f(t)$ can be arbitrary, one must relax the assumption that the units are prepared in the same initial state. Thus, only in this subsection, we allow $\rho_{U_{n}}(n \delta t) \neq \rho_{U_{m}}(m \delta t)$ for $n \neq m$ [we set the initial time to be zero such that $\rho_{U_{n}}(n \delta t)$ denotes the initial state of the unit just before the interaction]. The incoming units are, however, still assumed to be decorrelated. The time evolution of the system is given by

$$
\begin{aligned}
\rho_{S}(n \delta t+\delta t)= & \operatorname{tr}_{U}\left[e^{-i\left(H_{0}+A F+H_{U}\right) \delta t} \rho_{S}(n \delta t) \rho_{U_{n}}(n \delta t)\right. \\
& \left.\times e^{i\left(H_{0}+A F+H_{U}\right) \delta t}\right] .
\end{aligned}
$$

By expanding Eq. (134) to first order in $\delta t$, we arrive at

$$
\rho_{S}(n \delta t+\delta t)=\rho_{S}(n \delta t)-i \delta t\left[H_{0}+\langle F\rangle_{U_{n}}(n \delta t) A, \rho_{S}(n \delta t)\right] .
$$

We now choose the state of the unit such that

$$
\langle F\rangle_{U_{n}}(n \delta t) \equiv \operatorname{tr}_{U_{n}}\left\{F \rho_{U_{n}}(n \delta t)\right\}=f(n \delta t) .
$$

Under these circumstances, we obtain from Eq. (135), after rearranging the terms in the limit $\delta t \rightarrow 0$,

$$
d_{t} \rho_{S}(t)=-i\left[H_{0}+f(t) A, \rho_{S}(t)\right],
$$

which is the desired evolution according to the Hamiltonian (133).

In fact, if $\delta t$ is chosen small enough compared to any other time scales, one could even include an additional reservoir in the description. In this case, the Hamiltonian to be simulated becomes

$$
H_{S R}(t)=H_{0}+f(t) A+V_{S R}+H_{R},
$$

and the joint system-reservoir state evolves according to

$$
d_{t} \rho_{S R}(t)=-i\left[H_{S R}(t), \rho_{S R}(t)\right] .
$$

\section{Thermodynamics}

In order to establish the thermodynamics of the present setup, we need to consider how the units change over time. Similarly as for Eqs. (134) and (135), we deduce that

$$
\begin{aligned}
\rho_{U_{n}}(n \delta t+\delta t)= & \rho_{U_{n}}(n \delta t)-i \delta t\left[H_{U}\right. \\
& \left.+\langle A\rangle_{S}(n \delta t) F, \rho_{U_{n}}(n \delta t)\right] .
\end{aligned}
$$

Since the unit state changes unitarily, the entropy change of the units is zero, $d_{t} S_{U_{n}}(t)=0$, and hence, according to the classification schemes from Sec. IV, the stream of units may behave as a work reservoir.

To confirm it, we now consider energy balances. First, the change in unit energy is given by

$$
\begin{aligned}
d_{t} E_{U_{n}} & =\delta t^{-1} \operatorname{tr}_{U_{n}}\left\{H_{U}\left[\rho_{U_{n}}(n \delta t+\delta t)-\rho_{U_{n}}(n \delta t)\right]\right\} \\
& =i\langle A\rangle_{S}^{n} \operatorname{tr}_{U_{n}}\left\{\left[F, H_{U}\right] \rho_{U_{n}}^{n}\right\} .
\end{aligned}
$$

For the last line, we used Eq. (140). Second, we need to consider the switching work stemming from the timedependent coupling of the system and unit. However, we have to remember that the units change for each interval. Naively applying the definition (44) for $W_{\text {sw }}$, which is only valid for identical units, would yield a wrong result. Considering the interval starting in $n \delta t$ and lasting $\delta t$, we find that 


$$
\begin{aligned}
\delta t \dot{W}_{\mathrm{sW}}= & \operatorname{tr}_{S U_{n+1}}\left\{A F \rho_{S}(n \delta t+\delta t) \rho_{U_{n+1}}(n \delta t+\delta t)\right\} \\
& -\operatorname{tr}_{S U_{n}}\left\{A F \rho_{S U_{n}}(n \delta t+\delta t)\right\} .
\end{aligned}
$$

This quantity represents the work required to switch on the interaction for the next unit state $\rho_{U_{n+1}}(n \delta t+\delta t)$ minus the work required to switch off the interaction for the actual unit state $\rho_{U_{n}}(n \delta t+\delta t)$ at the end of the interval. To evaluate it, we use Eq. (140) and

$$
\begin{aligned}
\rho_{S U_{n}}(n \delta t+\delta t)= & \rho_{S}(n \delta t) \rho_{U_{n}}(n \delta t) \\
& -i \delta t\left[H_{S}+H_{U}+A F, \rho_{S}(n \delta t) \rho_{U_{n}}(n \delta t)\right] \\
& -i \delta t \operatorname{tr}_{R}\left\{\left[V_{S R}, \rho_{S R}(n \delta t)\right]\right\} \rho_{U_{n}}(n \delta t),
\end{aligned}
$$

$$
\begin{aligned}
\rho_{S}(n \delta t+\delta t)= & \rho_{S}(n \delta t)-i \delta t\left[H_{S}+f(n \delta t) A, \rho_{S}(n \delta t)\right] \\
& -i \delta t \operatorname{tr}_{R}\left\{\left[V_{S R}, \rho_{S R}(n \delta t)\right]\right\},
\end{aligned}
$$

which follow from the Liouville-von Neumann equation for a time step $\delta t$. After a straightforward but tedious calculation, we arrive at (to first order in $\delta t$ )

$$
\begin{aligned}
\dot{W}_{\mathrm{sw}}= & f^{\prime}(n \delta t)\langle A\rangle_{S}(n \delta t) \\
& +i\langle A\rangle_{S}(n \delta t) \operatorname{tr}_{U_{n}}\left\{\left[F, H_{U}\right] \rho_{U_{n}}(n \delta t)\right\} .
\end{aligned}
$$

Here, we introduced the discretized derivative of $f(t)$ as

$$
f^{\prime}(n \delta t) \equiv \frac{f(n \delta t+\delta t)-f(n \delta t)}{\delta t},
$$

which is well behaved because we assumed $f(t)$ to be differentiable. Since the units act as a pure work reservoir, the total rate of work done on the system can be defined as

$$
\dot{W} \equiv \dot{W}_{\mathrm{sw}}-d_{t} E_{U_{n}}=f^{\prime}(n \delta t)\langle A\rangle_{S}(n \delta t) .
$$

Finally, let us compare this result with our general treatment from Sec. II A, where we found that for a time-dependent Hamiltonian of the form (138), the rate of work done on the system is [compare with Eq. (6)]

$\dot{W}(t)=\operatorname{tr}_{S R}\left\{\rho_{S R}(t) d_{t} H_{\text {tot }}(t)\right\}=\frac{d f(t)}{d t} \operatorname{tr}_{S}\left\{A \rho_{S}(t)\right\}$.

This expression exactly coincides for $t=n \delta t$ with Eq. (147). This confirms that there is a clean (but somewhat artificial) way to simulate a driven system by a stream of units and to justify that the driving corresponds to a pure work source.

\section{APPLICATIONS}

In this section, we demonstrate the use of the repeated interaction framework that we have developed in the previous sections by considering specific examples. We mostly emphasize the way in which the setups can be analyzed from our thermodynamic perspective and refer to the original literature for more details. Many more setupse.g., the measurement and feedback scheme realized in Ref. [34], the squeezed reservoirs of Ref. [27], the coherent states of Ref. [25], or the entangled unit states of Ref. [23,172]—can be analyzed within our framework but will not be considered here for brevity.

\section{A. Information reservoir: Mandal-Jarzynski engine}

We start by providing a microscopic model describing the information-driven engine first proposed by Mandal and Jarzynski [35] and show that it falls within the class of information reservoirs considered in Sec. IV C.

The system is modeled as a three-level system with Hamiltonian

$$
H_{S}=\epsilon_{S}(|A\rangle\langle A|+| B\rangle\langle B|+| C\rangle\langle C|),
$$

and the unit is modeled by a two-level system ("bit") with Hamiltonian

$$
H_{U}=\epsilon_{U}(|0\rangle\langle 0|+| 1\rangle\langle 1|) .
$$

Thus, the bare system and unit Hamiltonians are completely degenerate. The system-unit interaction is switched on and off at the beginning and end of the full interval of length $\tau$, such that the time interval between the unit interactions is infinitely short (i.e., $\tau^{\prime} \rightarrow \tau$ ). During the interaction, the degeneracy of the system and unit states is lifted via

$$
V_{S U}=\frac{\Delta w}{\epsilon_{S}} H_{S}|1\rangle\langle 1|,
$$

such that the energy of the system-unit states $|A 1\rangle,|B 1\rangle$, $|C 1\rangle$ becomes $\epsilon_{S}+\epsilon_{U}+\Delta w$, and the energy of the systemunit states $|A 0\rangle,|B 0\rangle,|C 0\rangle$ is $\epsilon_{S}+\epsilon_{U}$.

The model is completed by adding a weakly coupled reservoir and by assuming that it induces thermally activated transitions between the following levels: $A 0 \leftrightarrow B 0$, $B 0 \leftrightarrow C 0, C 0 \leftrightarrow A 1, A 1 \leftrightarrow B 1$, and $B 1 \leftrightarrow C 1$. For other possible physical setups, see Refs. [100,102].

Note that, although we have written down the model in a quantum mechanical way, the model in Ref. [35] is purely classical. In this spirit, we neglect any subtleties arising from deriving a $\mathrm{ME}$ for degenerate quantum systems and use a classical rate equation where those levels that interact with the reservoir are connected, as specified above. Then, the ME takes the form $d_{t} \mathbf{p}(t)=\mathcal{R} \mathbf{p}$ with (in suitable units) [35] 


$$
\begin{gathered}
\mathbf{p}(t)=\left(\begin{array}{c}
p_{A 0}(t) \\
p_{B 0}(t) \\
p_{C 0}(t) \\
p_{A 1}(t) \\
p_{B 1}(t) \\
p_{C 1}(t)
\end{array}\right), \\
\mathcal{R}=\left(\begin{array}{cccccc}
-1 & 1 & 0 & 0 & 0 & 0 \\
1 & -2 & 1 & 0 & 0 & 0 \\
0 & 1 & -2+\epsilon & 1+\epsilon & 0 & 0 \\
0 & 0 & 1-\epsilon & -2-\epsilon & 1 & 0 \\
0 & 0 & 0 & 1 & -2 & 1 \\
0 & 0 & 0 & 0 & 1 & -1
\end{array}\right) .
\end{gathered}
$$

The parameter $\epsilon \in(-1,1)$ is related to $\Delta w$ by local detailed balance [see Eq. (30)] via $-\beta \Delta w=\ln [(1-\epsilon) /(1+\epsilon)]$. The initial state of the incoming units is given by $\rho_{U}(0)=$ $[(1+\delta) / 2]|0\rangle\langle 0|+[(1-\delta) / 2]| 1\rangle\langle 1|$ with $\delta \in[-1,1]$. The stationary solution for the system is obtained by solving this rate equation for a time $\tau$ with an initial condition $\rho_{U}(0)$ for the units and $\rho_{S}(0)=\rho_{S}(\tau)$ for the system. As demonstrated in Ref. [35], it can be solved exactly.

Our thermodynamic analysis from Sec. III now tells us immediately that $\Delta E_{S}=0$ and $\Delta E_{U}=0$ due to the degeneracy of the bare system and unit Hamiltonian. Since $\Delta E_{U}=0$, it also follows from Eq. (61) that the stream of bits constitutes an information reservoir unless $\Delta S_{U}=0$, too. Furthermore, because we have no explicit driving, we also have $W_{X}=0$ [see Eq. (43)], and when the system has reached its steady state, we will also have $\Delta S_{S}=0$. The first law (46) then tells us that

$$
Q=-W_{\mathrm{sw}}
$$

In fact, Mandal and Jarzynski imagined a little load attached to the system such that the heat absorbed from the system can be identified as work. Within our setup, we indeed see that this "attaching of the load," i.e., the switching on and off of the system-unit interaction, is responsible for providing that energy and has a clear microscopic interpretation in our framework. From Eq. (44), we can immediately compute

$$
W_{\mathrm{sw}}=-\Delta w\left(\left\langle 1\left|\rho_{U}(\tau)\right| 1\right\rangle-\left\langle 1\left|\rho_{U}(0)\right| 1\right\rangle\right) .
$$

This term exactly equals (minus) the work identified in Ref. [35]. Finally, in accordance with our second law (62), it was also shown in Ref. [35] that

$$
\beta Q=-\beta W_{\mathrm{sw}} \leq \Delta S_{U}
$$

\section{B. Work reservoir: Micromaser}

The micromaser is historically, experimentally, and theoretically important, and its operation is based on repeated interactions. Beyond quantum optics, it has also been used, e.g., as a model system for transport in superconducting single-electron transistors coupled to quantum resonators [173], where it displays an intriguing dynamics such as selfsustained oscillations and transitions to multistable behavior. We now briefly elaborate on the fact that in its simplest version, the micromaser can be viewed as a system interacting with a stream of units that operate as a work reservoir. Our approach will be qualitative since the detailed calculations can be found, e.g., in Refs. [32,33,165].

The system $S$ in this case consists of a high-quality cavity supporting a microwave field with Hamiltonian $H_{S}=\Omega a^{\dagger} a$, where $a^{\dagger}$ and $a$ are bosonic creation and annihilation operators and $\Omega$ denotes the frequency of the cavity. The microwave mode is coupled to an external reservoir of electromagnetic field modes at equilibrium with temperature $\beta^{-1}$ and "high quality" means that the coupling is very weak, especially such that it is negligible on time scales when the system interacts with an atom flying through the cavity. This atom corresponds to a unit $U$ and can be conveniently modeled as a two-level system (TLS) $H_{U}=(\Delta / 2)(|1\rangle\langle 1|-| 0\rangle\langle 0|)$ with energy gap $\Delta \approx$ $\Omega$ (on resonance with the cavity). Depending on the experimental details, the units can be prepared in different states and might arrive at the cavity at regular intervals or Poisson distributed; see Fig. 6 for a sketch of the setup and Ref. [34] for a recent experiment involving measurement and feedback.

In the standard setup, the TLSs are prepared in a statistical mixture of excited and ground states, and they interact with the cavity during a short time (compared to the cavity lifetime) via a Jaynes-Cummings interaction

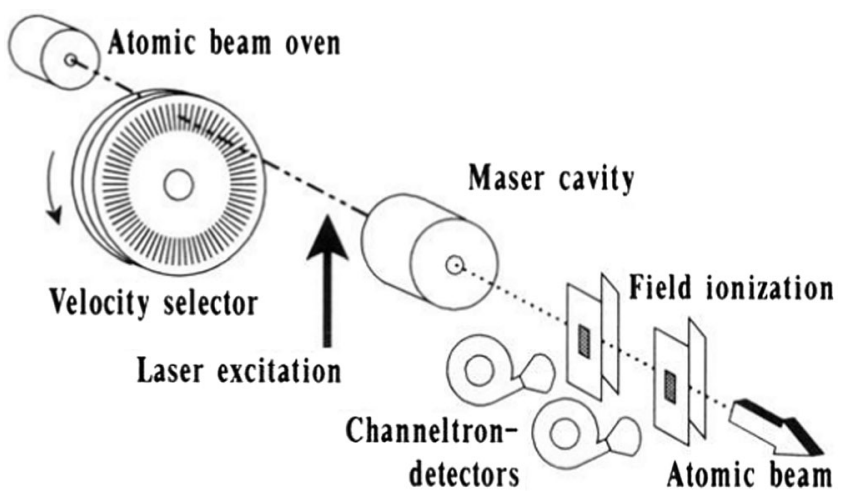

FIG. 6. Sketch of a micromaser setup. The units are produced in an atomic beam oven and are initialized with a velocity selector and laser excitation. They then interact with a microwave ("maser") cavity (the system) before they are finally detected and their state is read out. The figure is taken from Ref. [174], reporting on experimental observation of sub-Poissonian photon statistics in a micromaser. 
Hamiltonian $\quad V_{S U}=g\left(|0\rangle\left\langle 1\left|a^{\dagger}+\right| 1\right\rangle\langle 0| a\right) \quad$ causing the atom to emit or absorb photons [33]. By tuning the interaction time (or the interaction strength $g$, respectively) correctly, one can make sure that an atom initially in the excited (ground) state will always emit (absorb) a cavity photon. In essence, the effect of the cavity is therefore to swap the population of the TLSs.

This implies a difference in energy between incoming and outgoing TLSs $\left(\Delta E_{U} \neq 0\right)$ but not in entropy because entropy is invariant under the exchange of state labels $\left(\Delta S_{U}=0\right)$. Hence, the stream of atoms acts as a pure source of work, which builds up a photon field inside the cavity. However, because the cavity is weakly coupled to an outside thermal reservoir, it constantly loses photons, too. To achieve a steady-state occupation of the cavity above the thermal average, the incoming TLSs must be in a population-inverted state, i.e., have a higher probability to be excited than in the ground state. More details, such as the exact threshold condition for a buildup of the cavity field, are given in Refs. [32,33,165].

\section{Quantum coherence as a resource: Lasing without inversion}

As explained in the previous section, the thermodynamic working principle (but not the physical origin) of the micromaser can be understood within classical physics. However, it is also possible to populate a cavity above its thermal distribution by using a stream of atoms (units) without population inversion. This phenomenon is known as lasing without inversion [33] and results from a destructive interference of the photon absorption process due to a coherent superposition of the energy levels of the incoming unit. Thus, lasing without inversion is a pure quantum effect.

The idea to use quantum coherence via lasing without inversion in order to extract more work from a heat engine than classically possible was proposed by Scully et al. in Ref. [22]; see also Refs. [175,176] for similar models and Ref. [82] for a resource theory formulation of the problem. We now briefly sketch how to treat lasing without inversion [33] and how to rederive the results from Ref. [22] from our results of Sec. VB.

The system we are considering is the same as for the micromaser (i.e., a single cavity with frequency $\Omega$ ). The units are three-level systems described by

$$
H_{U}=E_{a}|a\rangle\left\langle a\left|+E_{b}\right| b\right\rangle\left\langle b\left|+E_{c}\right| c\right\rangle\langle c| .
$$

We assume a so-called " $\Lambda$ configuration" [33] where the two states $|b\rangle$ and $|c\rangle$ are nearly degenerate $\left(E_{b} \approx E_{c}\right)$ and well separated from the excited state $|a\rangle$, which is nearly resonant with the cavity (i.e., $\Omega \approx E_{a}-E_{b} \approx E_{a}-E_{c}$ ). The initial state of the units is modeled as a statistical mixture between the energy eigenstates with an additional coherence allowed between the near-degenerate levels $|b\rangle$ and $|c\rangle$. Following the notation of Ref. [22], we use

$$
\begin{aligned}
\rho_{U}= & P_{a}|a\rangle\left\langle a\left|+P_{b}\right| b\right\rangle\left\langle b\left|+P_{c}\right| c\right\rangle\langle c| \\
& +\rho_{b c}|b\rangle\left\langle c\left|+\rho_{c b}\right| c\right\rangle\langle b|,
\end{aligned}
$$

with $P_{a}+P_{b}+P_{c}=1, P_{a, b, c} \geq 0$, and $\rho_{b c}=\rho_{c b}^{*} \in \mathbb{C}$. Beyond that, the positive definiteness of $\rho_{U}$ requires $\rho_{b c} \rho_{c b} \leq P_{b} P_{c}$. Finally, the interaction between system and unit is modeled by a generalized Jaynes-Cummings Hamiltonian,

$\tilde{V}=-g\left[a(|a\rangle\langle b|+| a\rangle\langle c|)+a^{\dagger}(|b\rangle\langle a|+| c\rangle\langle a|)\right]$,

where we assumed that the direct transition between $|b\rangle$ and $|c\rangle$ is dipole forbidden [33] and assumed an interaction scenario as in Sec. V B 1. We note that in the interaction picture with respect to $H_{S}+H_{U}$, we have

$$
\tilde{V}_{\text {int }}(t) \equiv e^{i\left(H_{S}+H_{U}\right) t} V_{S U} e^{-i\left(H_{S}+H_{U}\right) t} \approx \tilde{V}
$$

because of the resonance condition.

We now assume a cavity of very high quality, neglecting any dissipation due to the electromagnetic reservoir such that the system interacts with many atoms coherently. This corresponds to the stage $1 \rightarrow 2$ in Fig. 2 of Ref. [22]. Using Eq. (158), it is easy to confirm that $\operatorname{tr}_{U}\left\{\tilde{V} \rho_{U}\right\}=0$. Then, following Sec. V B, we see that Eq. (125) requires us to compute 16 correlations functions $\left\langle B_{l} B_{k}\right\rangle_{U}$, where we identify $B_{1} \equiv-g|a\rangle\left\langle b\left|, \quad B_{2} \equiv-g\right| a\right\rangle\left\langle c\left|, \quad B_{3} \equiv-g\right| b\right\rangle\langle a|$, and $B_{4} \equiv-g|c\rangle\langle a|$. Only six are nonzero, and the ME (125) in the interaction picture becomes

$d_{t} \rho_{S}(t)=\gamma_{\text {eff }}\left\{2 P_{a} \mathcal{D}\left[a^{\dagger}\right]+\left(P_{b}+P_{c}+\rho_{b c}+\rho_{c b}\right) \mathcal{D}[a]\right\} \rho_{S}(t)$,

with the dissipator $\mathcal{D}$ defined below Eq. (93) and some effective and, for our purposes, unimportant rate $\gamma_{\text {eff }}>0$. The Lindblad form is ensured by the nonnegativity of the unit density matrix, which implies $\left(P_{b}+P_{c}+\rho_{b c}+\rho_{c b}\right) \geq 0$. Note that $\mathcal{D}\left[a^{\dagger}\right]$ describes the absorption and $\mathcal{D}[a]$ the emission of a cavity photon.

If the unit is initially in a thermal state with occupations $P_{a}=e^{-\beta \Omega / 2} / Z$ and $P_{b}=P_{c}=e^{\beta \Omega / 2} / Z$ and without coherences, $\rho_{b c}=\rho_{c b}=0$, the rates for emission and absorption satisfy local detailed balance and the equilibrium state $\rho_{\beta}^{S}$ is the steady state of the ME.

In contrast, with coherences $\rho_{b c}+\rho_{c b}=2 \Re\left(\rho_{b c}\right)$ (which can be positive as well as negative), the rate of absorption can be decreased or increased. If $2 \Re\left(\rho_{b c}\right)<0$, the rate of photon absorption by the units is lowered, and lasing without inversion becomes possible. Note that, typically, such coherences in three-level lambda systems are created by two coherent light fields via stimulated 
Raman processes, i.e., time-dependent external lasers that thus ultimately provide a resource of energy.

To show the equivalence of these results with those of Ref. [22], we compute the evolution of the mean photon occupation of the cavity $N(t) \equiv \operatorname{tr}_{S}\left\{a^{\dagger} a \rho_{S}(t)\right\}$. Using $\left[a, a^{\dagger}\right]=1$ and the property $\operatorname{tr}\{A B C\}=\operatorname{tr}\{C A B\}$, we obtain

$d_{t} N(t)=\gamma_{\mathrm{eff}}\left\{2 P_{a}[1+N(t)]-\left(P_{b}+P_{c}+\rho_{b c}+\rho_{c b}\right) N(t)\right\}$,

which is identical to Eq. (5) in Ref. [22] [after replacing $N(t)$ with $n_{\phi}$ and $\gamma_{\text {eff }}$ with $\alpha$ ] from which the results in Ref. [22] are derived. The steady-state occupation reads

$$
\begin{aligned}
N_{\text {eff }} \equiv \lim _{t \rightarrow \infty} N(t) & =\frac{2 P_{a}}{P_{b}+P_{c}-2 P_{a}+2 \Re\left(\rho_{b c}\right)} \\
& =\left(e^{\beta \Omega}-1+e^{\beta \Omega / 2} Z \Re\left(\rho_{b c}\right)\right)^{-1} .
\end{aligned}
$$

For zero coherence, it corresponds to the (equilibrium) Bose distribution. But for finite coherence, the cavity population can be lowered or raised. This means that the nonequilibrium free energy of the incoming atoms has been converted into a nonequilibrium free energy for the cavity. This feature alone does not yet yield a positive work output, but a thermodynamic cycle that does so is presented in Ref. [22]. It basically relies on the fact that the population $N(t)$ of the cavity is related to a radiation pressure $P$ via $P \sim N(t)$, which can be used to drive a piston. Then, by putting the cavity first in contact with the stream of atoms populating it according to some effective temperature $T_{\text {eff }}>T$ [which can be inferred from Eq. (163)] and afterwards with a standard heat reservoir at temperature $T$, we can extract work proportional to $N_{T_{\text {eff }}}-N_{T}$, where $N_{T}$ denotes the Bose distribution.

The idea behind lasing without inversion thus provides an example of how our framework can account for coherences. Following Ref. [22], we have shown that these latter can be used as a thermodynamic resource. During the first part of the cycle as described above, the units do not correspond to any limiting classification scheme introduced in Sec. IV because the initial state of the units is not thermal; in general, the energy as well as the entropy of the units will change, whereas during the rest of the cycle (where the system expands back to an equilibrium distribution), there are no units interacting with the system.

\section{Measurement and feedback: Electronic Maxwell demon}

In the traditional thought-experiment of Maxwell, the demon shuffles gas particles from a cold to a hot reservoir with negligible consumption of energy $[18,19]$. In an isothermal setup, a similar violation of the traditional second law appears if a feedback mechanism shuffles particles from a reservoir with low chemical potential to a reservoir with high chemical potential. This is the central idea of the electronic Maxwell demon, which has been theoretically well studied for a number of different models [102,151,155,177-182]. The setup proposed in Ref. [155] was recently experimentally realized in Ref. [183]. Below, we revisit one particular electronic Maxwell demon.

The system to be controlled is a conventional singleelectron transistor (SET), which consists of a single-level quantum dot connected to two thermal reservoirs with chemical potential $\mu_{\nu}(\nu \in\{L, R\})$ at the same inverse temperature $\beta$. The quantum dot can either be filled with an electron of energy $\epsilon_{S}$ or empty (corresponding to a zeroenergy state). A sketch of the setup (with the feedback mechanism described below) is shown in Fig. 7. The ME governing the time evolution of the system in the absence of feedback is

$$
d_{t} \rho_{S}(t)=\sum_{\nu} \mathcal{L}_{\beta}^{(\nu)}\left(\Gamma_{\nu}\right) \rho_{S}(t)
$$

where the thermal generators are defined as

$\mathcal{L}_{\beta}^{(\nu)}\left(\Gamma_{\nu}\right) \equiv \Gamma_{\nu}\left\{\left(1-f_{\nu}\right) \mathcal{D}\left[|E\rangle_{S}\langle F|\right]+f_{\nu} \mathcal{D}\left[|F\rangle_{S}\langle E|\right]\right\}$.

Here, $f_{\nu}=\left(e^{\beta\left(\epsilon_{S}-\mu_{\nu}\right)}+1\right)^{-1}$ is the Fermi function evaluated at the energy of the quantum dot, and $\Gamma_{\nu} \geq 0$ is a bare rate that depends on the details of the microscopic-coupling Hamiltonian. Furthermore, $|E\rangle_{S}\left(|F\rangle_{S}\right)$ denotes the empty (filled) state of the dot and the dissipator $\mathcal{D}$ is defined in the same way as below Eq. (93).

We now engineer a demon mechanism operating a feedback control on the system which modulates the energy barriers of the dot (i.e., the bare rates $\Gamma_{\nu}$ ) depending on the

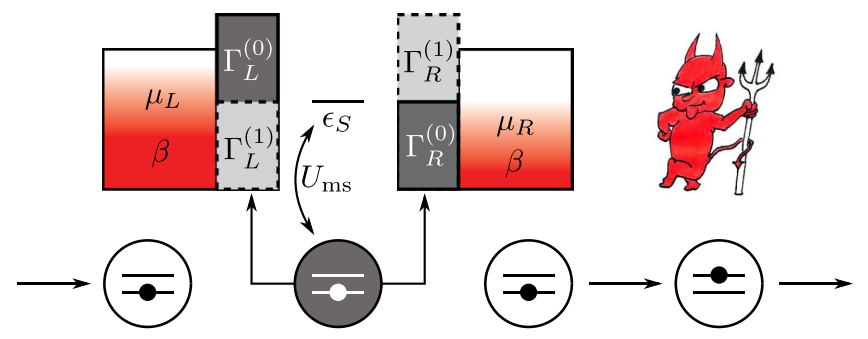

FIG. 7. Sketch of the electronic Maxwell demon. The system consists of a single-level quantum dot tunnel coupled to two electronic reservoirs with chemical potentials $\mu_{L} \geq \mu_{R}$ and inverse temperature $\beta$. The demon mechanism is implemented by a stream of units which monitors the state of the dot and, depending on its state, changes the tunneling barriers $\Gamma_{\nu}$ to make electronic transport against the bias possible. In the absence of the demon mechanism, the tunneling barriers would not depend on the state of the dot, and electrons would flow from left to right. The picture of the demon was provided courtesy of Ania Brzezinska. 
dot state as sketched in Fig. 7. The phenomenological description of this mechanism was done in Ref. [178] and its thermodynamical analysis was performed in Ref. [151]. A physical mechanism autonomously implementing this feedback was proposed in Ref. [155]. It relies on a capacitive coupling to another single-level quantum dot at a different temperature. This mechanism was further analyzed in Refs. [157,182] and will also be used below for comparison. We now propose a different mechanism, implementing the same feedback on the system. As sketched in Fig. 7, this one is based on repeated interactions with a stream of units consisting of two-level systems prepared in the state $\rho_{U}=(1-\epsilon) \Pi_{0}+\epsilon \Pi_{1}$, where $\Pi_{i}=$ $|i\rangle_{U}\langle i|$ is the projector on the state $i \in\{0,1\}$ and $\epsilon \in[0,1]$ is a free parameter quantifying the measurement error.

At the beginning of the interaction interval, we assume that the interaction produces an instantaneous unitary operation $U_{\mathrm{ms}}=\Pi_{E} \otimes \mathbf{1}_{U}+\Pi_{F} \otimes \sigma_{U}^{x}$, where $\Pi_{E}=$ $|E\rangle_{S}\langle E|$ and $\Pi_{F}=|F\rangle_{S}\langle F|$ and $\sigma_{U}^{x}=|0\rangle_{U}\langle 1|+| 1\rangle_{U}\langle 0|$, which can be interpreted as a measurement. Indeed, considering an initial system state of the form $\rho_{S}(t)=$ $p_{E}(t) \Pi_{E}+p_{F}(t) \Pi_{F}$, where $p_{E}(t)\left[p_{F}(t)\right]$ is the probability to find the system in the empty [filled] state, the postmeasurement state of the system and unit reads

$$
\begin{aligned}
\rho_{S U}^{\mathrm{ms}}(t) & =(1-\epsilon) p_{E}(t) \Pi_{E} \otimes \Pi_{0}+\epsilon p_{E}(t) \Pi_{E} \otimes \Pi_{1} \\
& +(1-\epsilon) p_{F}(t) \Pi_{F} \otimes \Pi_{1}+\epsilon p_{F}(t) \Pi_{F} \otimes \Pi_{0} .
\end{aligned}
$$

Note that $\epsilon=0$ corresponds to a perfect measurement in which the state of the unit after the measurement is 0 (1) if and only if the state of the system is $E(F)$. The reduced state of the system is always given by $\rho_{S}^{\mathrm{ms}}(t)=$ $\operatorname{tr}_{U}\left\{\rho_{S U}^{\mathrm{ms}}(t)\right\}=\rho_{S}(t)$, which means that the measurement does not disturb the system. This feature circumvents the difficulty mentioned in Sec. V B 1 and allows us to consider a continuously measured system in which $\delta t$ (the waiting time between two units) can be arbitrarily small despite the fact that the system is still interacting with its reservoir.

Next, in the spirit of Sec. IV E and Eq. (73), we postulate a Hamiltonian of the form $H_{\mathrm{fb}}=\epsilon_{S}|F\rangle_{S}\langle F|+\Pi_{0} H_{S R}^{(0)}+$ $\Pi_{1} H_{S R}^{(1)}+H_{R}$, which acts during the remaining interaction time $\delta t$ and changes the system reservoir coupling $H_{S R}^{(i)}$ according to the state $i$ of the unit. Assuming that we can treat $H_{S R}^{(i)}$ as a weak perturbation, we are effectively changing the tunneling rates from $\Gamma_{\nu}$ to $\Gamma_{\nu}^{(i)}$, and the time evolution of the system and unit is then given by

$\rho_{S U}(t+\delta t)=\sum_{i \in\{0,1\}} \exp \left\{\mathcal{L}_{\beta}^{(\nu)}\left(\Gamma_{\nu}^{(i)}\right) \delta t\right\} \Pi_{i} \rho_{S U}^{\mathrm{ms}}(t) \Pi_{i}$.

Tracing over the unit space and expanding this equation to first order in $\delta t$ (assuming that $\delta t$ is sufficiently small) yields an effective evolution equation for the system of the form $d_{t} \rho_{S}(t)=\mathcal{L}_{\text {eff }} \rho_{S}(t)$, with

$$
\begin{aligned}
\mathcal{L}_{\text {eff }} \equiv & \sum_{\nu} \mathcal{L}_{\text {eff }}^{(\nu)} \\
= & \sum_{\nu}\left[(1-\epsilon) \Gamma_{\nu}^{(1)}+\epsilon \Gamma_{\nu}^{(0)}\right]\left(1-f_{\nu}\right) \mathcal{D}\left[|E\rangle_{S}\langle F|\right] \\
& +\sum_{\nu}\left[(1-\epsilon) \Gamma_{\nu}^{(0)}+\epsilon \Gamma_{\nu}^{(1)}\right] f_{\nu} \mathcal{D}\left[|F\rangle_{S}\langle E|\right] .
\end{aligned}
$$

This equation is identical to the ME obtained for the system when it is subjected to the capacitive demon mechanism considered in Ref. [155], which results from coarse graining the demon dot and only retaining the SET degrees of freedom. In the error-free case $(\epsilon=0)$, it also reduces to the effective ME of Ref. [178], but the above procedure constitutes an elegant way to generalize arbitrary piecewise-constant feedback schemes [184] to finite detection errors.

We now turn to the thermodynamic analysis of our new demon mechanism. First of all, we can assume that the unit Hamiltonian $H_{U} \sim \mathbf{1}_{U}$ is fully degenerate. This implies $d_{t} E_{U}=0$ at all times. Then, during the measurement step, the system and unit correlate such that [see Eq. (68)] $I_{S: U}^{\mathrm{ms}}=d_{t} S_{U}^{\mathrm{ms}} \delta t$, where we used the fact that $d_{t} S_{S}^{\mathrm{ms}}=0$ since the system density matrix is left unchanged by the measurement. This correlation can then be exploited during the feedback step. The second law for feedback (75) in our situation reads, in a differential form, as

$$
\dot{\Sigma}_{S}^{\mathrm{fb}}=\beta\left(\mu_{L}-\mu_{R}\right) I_{L}+\frac{I_{S: U}^{\mathrm{ms}}}{\delta t} \geq \frac{I_{S: U}^{\mathrm{fb}}}{\delta t} \geq 0,
$$

where, for simplicity, we assumed that the system operates at steady state $d_{t} S_{S}^{\mathrm{fb}}=0$ and where we used $d_{t} S_{U}^{\mathrm{fb}}=0$ because the entropy of the unit does not change during the feedback step. Furthermore, the entropy flow reads $-\beta \dot{Q}^{(L)}-\beta \dot{Q}^{(R)}=\beta\left(\mu_{L}-\mu_{R}\right) I_{L}$, where $I_{L}$ is the matter current that entered the system from the left reservoir. Finally, $I_{S: U}^{\mathrm{fb}}$ quantifies the remaining system-unit correlations after the feedback step.

In the spirit of Eq. (47), it will turn out to be useful to include the final correlation $I_{S: U}^{\mathrm{fb}}$ in the second law and to define

$\dot{\Sigma}=\beta\left(\mu_{L}-\mu_{R}\right) I_{L}+\mathcal{I} \geq 0, \quad \mathcal{I} \equiv \frac{I_{S: U}^{\mathrm{ms}}-I_{S: U}^{\mathrm{fb}}}{\delta t}$.

Here, the newly defined quantity $\mathcal{I}$ is the rate with which we use up the correlations established during the measurement. Note that $\mathcal{I}$ had not yet been considered in previous works on this system. In fact, the information current in Ref. [151] is purely phenomenological in nature, whereas the information flow in Ref. [157] describes the same quantity but in a bipartite setting. Note that while both 
terms $\left[\left(I_{S: U}^{\mathrm{ms}}\right) / \delta t\right]$ and $\left[\left(I_{S: U}^{\mathrm{fb}}\right) / \delta t\right]$ would diverge when $\delta t \rightarrow 0, \mathcal{I}$, in general, remains finite, which motivates the use of Eq. (170) instead of Eq. (169). More specifically, we can compute

$$
\begin{aligned}
\mathcal{I}= & (1-\epsilon) \sum_{\nu}\left\{\Gamma_{\nu}^{(0)} f_{\nu} p_{E}+\Gamma_{\nu}^{(1)}\left(1-f_{\nu}\right) p_{F}\right\} \ln \frac{1-\epsilon}{\epsilon} \\
& -\epsilon \sum_{\nu}\left\{\Gamma_{\nu}^{(0)}\left(1-f_{\nu}\right) p_{F}+\Gamma_{\nu}^{(1)} f_{\nu} p_{E}\right\} \ln \frac{1-\epsilon}{\epsilon} \\
= & 2 \Gamma(1-2 \epsilon) \arctan (1-2 \epsilon) \\
& \times\left(\cosh \delta-(1-2 \epsilon) \sinh \delta \tanh \frac{\beta V}{4}\right) .
\end{aligned}
$$

For the last equality, we used the steady-state solution of $p_{E}$ and $p_{F}$ and parametrized the rates as $\Gamma_{L}^{(0)} \equiv \Gamma_{R}^{(1)} \equiv \Gamma e^{-\delta}$ and $\Gamma_{L}^{(1)} \equiv \Gamma_{R}^{(0)} \equiv \Gamma e^{\delta}$, such that $\delta \in \mathbb{R}$ characterizes a feedback strength. Note that $\mathcal{I}$ diverges for $\epsilon \rightarrow 0$. This makes sense because if we monitor the quantum dot in an error-free way, we can also extract an infinite amount of work by letting $\delta \rightarrow \infty$.

It is now interesting to compare the total entropy production generated by the two different electronic demon mechanisms, i.e., the one due to capacitive coupling with another quantum dot [155], denoted here as $\dot{\Sigma}^{\text {cap }}$, and the one generated by repeated interactions considered above, $\dot{\Sigma}$. These two entropy productions can then be compared to the effective one $\dot{\Sigma}_{S}^{\text {eff }}$, obtained when the demon mechanism is not known and the only information at hand is that of the system's effective description. The best one can do in this case is derive an "effective" second law in the spirit of Eq. (108) or of Ref. [151], which at the steady state can be written as

$$
\dot{\Sigma}_{S}^{\mathrm{eff}} \equiv \sum_{\nu} \operatorname{tr}_{S}\left\{\left(\mathcal{L}_{\mathrm{eff}}^{(\nu)} \bar{\rho}\right) \ln \bar{\rho}_{\nu}\right\} \geq 0
$$

and is equivalent to Eq. (14) of Ref. [155]. Here, $\bar{\rho}$ is the steady state fulfilling $\mathcal{L}_{\text {eff }} \bar{\rho}=0$ and $\bar{\rho}_{\nu}$ is the steady state with respect to reservoir $\nu$, i.e., $\mathcal{L}_{\text {eff }}^{(\nu)} \bar{\rho}_{\nu}=0$. Since this effective approach only quantifies the demon effect on the system and neglects the demon's dissipation, it will typically underestimate the true dissipation $[96,155]$.

To make the comparison between the various dissipations meaningful, we must compare them in the regime where they all give rise to the same effective dynamics on the system, i.e., to the same ME (168). For the repeated interaction mechanism, $\dot{\Sigma}$ is given by Eq. (170), while the effective dissipation $\dot{\Sigma}_{S}^{\text {eff }}$ is given by Eq. (172). For the capacitive mechanism, $\dot{\Sigma}^{\text {cap }}$ is given by Eq. (7) in Ref. [155] in the fast demon limit $\Gamma_{D} \rightarrow \infty$, which is required to derive the ME (168). In this limit, $\dot{\Sigma}^{\text {cap }}$ also coincides with Eq. (24) in Ref. [157] and therefore gives it an alternative
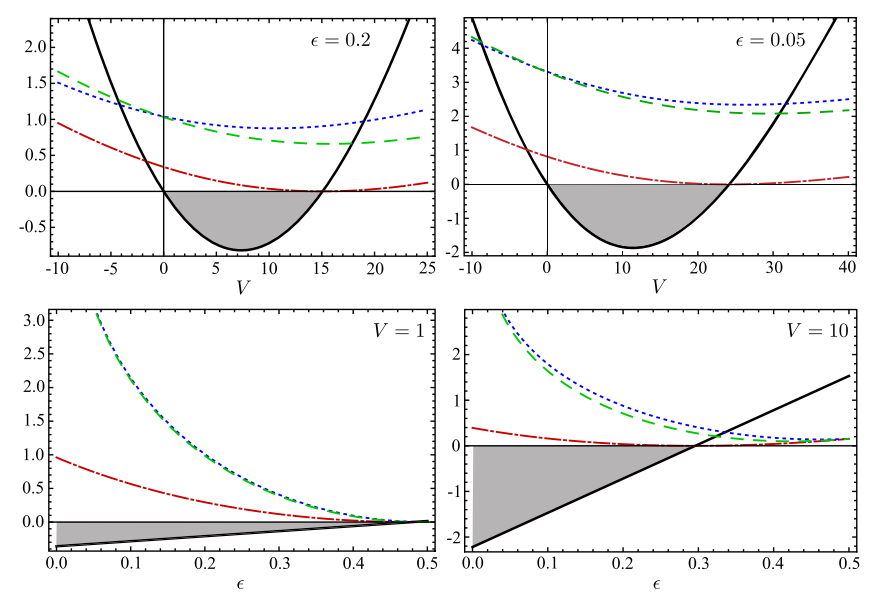

FIG. 8. Plot of the rate of chemical work $\beta\left(\mu_{L}-\mu_{R}\right) I_{L}$ (thick and solid black line) and of three different dissipations: the total one generated by the repeated interaction feedback mechanism $\dot{\Sigma}$ [dotted blue line, Eq. (170)], the total one generated by the capacitive feedback mechanism $\dot{\Sigma}^{\text {cap }}$ [dashed green line, Refs. [155,157]], and the "effective" estimate obtained in these two cases if the demon mechanisms were not known $\dot{\Sigma}_{S}^{\text {eff }}$ [dashdotted red line, Eq. (172) and Ref. [151]]. The top row shows these quantities as a function of the bias voltage $V \equiv \mu_{L}-\mu_{R}$ for two different measurement errors $\epsilon$. The bottom row shows them as a function of the measurement error $\epsilon \in[0,1 / 2)$ for two different voltages $V$. The region in which we extract work is shaded in gray. Other parameters are $\Gamma=1, \delta=\ln 2, \beta=0.1$, and $U=0.1$, and we choose a symmetric configuration of the bias, $\mu_{L}=\epsilon_{S}+V / 2$ and $\mu_{R}=\epsilon_{S}-V / 2$, effectively eliminating the dependence on $\epsilon_{S}$ in the equations.

interpretation in terms of the flow of mutual information. Note that we can link the measurement error $\epsilon$ to the parameters used in Ref. [157] via the relation $\beta_{D} U=$ $2 \ln [(1-\epsilon) / \epsilon]$. The comparison is done in Fig. 8. We observe that the effective second law $\dot{\Sigma}_{S}^{\text {eff }}$ greatly underestimates the true entropy production as expected, but also that the total entropy production generated by the two different demon mechanisms, $\dot{\Sigma}$ and $\dot{\Sigma}^{\text {cap }}$, is remarkably close.

\section{DISCUSSIONS AND CONCLUSIONS}

\section{A. Connection to traditional thermodynamics}

We have seen in Sec. IVA that if the units are initially thermal, our engine cannot surpass Carnot efficiency and deviations of the final unit state from the ideal thermal reservoir (i.e., nonequilibrium effects) always cause an even smaller efficiency. However, if the units are prepared in an arbitrary state, we found that one can continuously extract work from a single heat reservoir by lowering the nonequilibrium free energy of the units according to Eq. (49). We explained that this does not violate the second law of thermodynamics because the overall entropy does not decrease. One may nevertheless wonder if this 


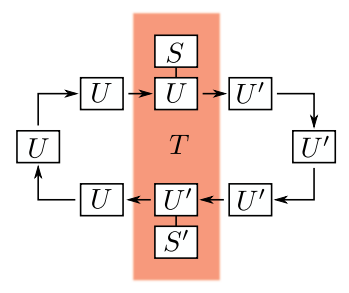

FIG. 9. Sketch of the setup to demonstrate the Kelvin-Planck statement of the second law: The outgoing units $U^{\prime}$ after the interaction with system $S$ are reset to the state $U$ via interaction with a second system $S^{\prime}$ coupled to the same thermal reservoir as $S$.

contradicts the classical formulation of the second law according to Kelvin and Planck, stating the following: "There is no cyclic process in nature whose sole result is the conversion of heat from a single reservoir into work".

To answer, let us close the "cycle of units" by feeding the outgoing units back into the system $S$ after they have interacted with an additional system $S^{\prime}$ in contact with the same overall heat reservoir at temperature $T$, as illustrated in Fig. 9. We assume to be at a steady state and denote the outgoing units after the interaction with system $S$ by $U^{\prime}$, which are, in turn, the incoming units for system $S^{\prime}$. The additional system $S^{\prime}$ is required to reset the units $U^{\prime}$ to the state $U$, which again correspond to the incoming units of $S$. Then, for $S$ and $S^{\prime}$ we find the two separate second laws (49),

$$
\begin{gathered}
\beta\left(W-\Delta F_{U}\right) \geq I_{S: U}(\tau) \geq 0, \\
\beta\left(W^{\prime}-\Delta F_{U^{\prime}}\right) \geq I_{S^{\prime}: U^{\prime}}(\tau) \geq 0,
\end{gathered}
$$

which, when added together and using $\Delta F_{U^{\prime}}=$ $F_{U}-F_{U^{\prime}}=-\Delta F_{U}$, lead to

$$
\beta\left(W+W^{\prime}\right) \geq I_{S: U}(\tau)+I_{S^{\prime}: U^{\prime}}(\tau) \geq 0 .
$$

Hence, although $W$ might be negative, the sum $W+W^{\prime}$ must be non-negative in perfect agreement with the KelvinPlanck statement of the second law of thermodynamics.

\section{B. Quantum vs classical thermodynamics}

At this point, it is worth revisiting the debated question of whether quantum thermodynamics offers advantages (e.g., in terms of a higher power output or efficiency) in comparison to classical thermodynamics. There is ample evidence that states with quantum properties such as entanglement, coherence, or squeezing can be used to extract more work than from thermal states; see, e.g., Refs. [22-25,27,28,82,175,176,185,186] (and also Sec. VIC). However, this by no means implies that quantum thermodynamics outperforms classical thermodynamics. Indeed, classical nonequilibrium properties are usually not considered, but if one only allows for nonequilibrium properties that are of a purely quantum origin, this amounts to an unfair competition. In the repeated interaction framework, the nonequilibrium free energy (23) captures both quantum and classical effects, and we will now use it to analyze the thermodynamics of work extraction. Note that, despite its limitations, the framework fully captures quantum effects in the unit state and in the system-unit interaction.

In the ideal scenario where no final system-unit correlations are present (since they always degrade the amount of extractable work) [187], the second law (49) bounds the extractable work as

$$
-W \leq-\Delta F_{U}=F_{U}(0)-F_{U}(\tau) .
$$

The extractable work $-W$ therefore gets maximized for a maximum initial and minimum final nonequilibrium free energy. The relevant question is therefore whether this procedure can be improved because of quantum effects?

Let us consider an arbitrary unit that has $N$ levels and a Hamiltonian $H_{U}=\sum_{n} E_{n}|n\rangle\langle n|$ with $E_{1} \leq \ldots \leq E_{N}$. The state with maximum free energy corresponds to a state with maximum energy and minimum entropy as we can easily infer from Eq. (23). This state is given by $\rho_{U}(0)=|N\rangle\langle N|$ and thus is also an allowed classical state [188]. Finding the state with minimum free energy is more tricky and, in general, context dependent [189]. We can nevertheless easily show that the state with minimum free energy must be "classical." For this purpose, let us define classical states as states that are diagonal in the energy eigenbasis, i.e., states that can be written as $\rho_{\mathrm{cl}}=\sum_{n} p_{n}|n\rangle\langle n|$. Let us denote by $\rho_{\mathrm{QM}}$ quantum states (i.e., states for which there exists $n \neq m$ such that $\left\langle n\left|\rho_{\mathrm{QM}}\right| m\right\rangle \neq 0$ ) which minimize the nonequilibrium free energy. Its corresponding classical state (obtained by neglecting all coherences) is given by $\rho_{\mathrm{cl}}=\sum_{n}\left\langle n\left|\rho_{\mathrm{QM}}\right| n\right\rangle|n\rangle\langle n|$. If we now assume that the nonequilibrium free energy corresponding to $\rho_{\mathrm{QM}}$ is strictly smaller than that of $\rho_{\mathrm{cl}}$, we get

$$
E\left(\rho_{\mathrm{QM}}\right)-E\left(\rho_{\mathrm{cl}}\right)<T\left[S\left(\rho_{\mathrm{QM}}\right)-S\left(\rho_{\mathrm{cl}}\right)\right] .
$$

By construction, we know that the left-hand side is zero since

$E\left(\rho_{\mathrm{QM}}\right)=\operatorname{tr}\left\{H_{U} \rho_{Q M}\right\}=\sum_{n} E_{n}\left\langle n\left|\rho_{\mathrm{QM}}\right| n\right\rangle=E\left(\rho_{\mathrm{cl}}\right)$.

Furthermore, since we have that $S\left(\rho_{\mathrm{QM}}\right)<S\left(\rho_{\mathrm{cl}}\right)$ (Theorem 11.9 in [43]), Eq. (177) leads to a contradiction, and the state of minimum free energy must necessarily be classical.

We thus proved that within our framework, there is no benefit in using quantum over classical "resources" in terms of the bound dictated by the generalized second law. That is 
to say, for any scenario where quantum effects are used to extract work, an equivalent classical scenario can be conceived (using classical units with the same number $N$ of basis states but a different interaction), which extracts at least the same amount of work. It should be noted, however, that we did not investigate the question of how this bound can be reached, and there is evidence that quantum systems can offer advantages in terms of speed, i.e., if we want to extract work at finite power [190-195].

\section{Summary and outlook}

We start by summarizing the paper together with its key results. In Sec. II, we reviewed the exact identities describing the correlated dynamics of two interacting systems, one of which could be considered as a reservoir that is initially thermal. We also considered the weakcoupling limit, which implicitly assumes macroscopic reservoirs. In Sec. III, we extended these concepts to describe a system that, in addition to continuously interacting with an initially thermal reservoir, is subjected to repeated interactions with identical units prepared in arbitrary states. By establishing exact energy and entropy balances, we showed that the stream of units can be seen as a nonequilibrium reservoir or a resource of free energy. In Sec. IV, we identified the limits where these units operate as a pure work, heat, or information reservoir and also formulated Landauer's principle. Most importantly, we showed that our setup can be used to formulate quantum feedback control, derived a new generalized bound for the extractable work, and provided a clean connection to the theory of information reservoirs.

Up to that point, the discussion was based on exact identities, which are conceptually powerful but of limited practical use. In Sec. V, we started focusing on limits where the system obeys a closed effective dynamics. We derived effective MEs for the system and established their corresponding thermodynamics: This has been done in Sec. VA when the system weakly and continuously interacts with a thermal reservoir while rarely interacting with units arriving at Poisson-distributed times, and in Sec. V B when the system frequently interacts solely with the units. We also discussed in Sec. VC the limit where a time-dependent system Hamiltonian can be effectively mimicked by a stream of units behaving as a pure work source.

Finally, in Sec. VI, we used our framework to analyze important models that were previously considered for their nonconventional thermodynamic features. In Sec. VI A, we proposed a microscopic model effectively implementing the Mandal-Jarzynski engine. We showed that the work extracted from the entropy of the tape originates from the switching on and off of the system-unit interaction. In Sec. VI B, we used our framework to study the thermodynamics of the micromaser, which is probably the most popular setup making use of repeated interactions. Building on an extension of this model, we showed in Sec. VIC that work can be extracted from purely quantum features based on the idea of "lasing without inversion." Finally, in Sec. VID, we considered a Maxwell demon effect on an electronic current crossing a single-level quantum dot that was theoretically studied in the past and also experimentally realized. We analyzed this effect thermodynamically when the demon mechanism operates by repeated interactions with a stream of units, and we showed how it differs from the previously considered mechanisms.

The framework of repeated interactions presented in this paper is quite general and provides a unifying picture for many problems currently encountered in the literature. It nevertheless has limitations. For instance, our results crucially rely on the fact that the individual units in the incoming stream are decorrelated. This assumption is often justified, but recent works started to investigate the role of correlated units, classically [196-200] as well as quantum mechanically [201]. In general, this leads to a refined second law with tighter bounds on the amount of extractable work [196,197,200]. The cost for creating these correlated units ("patterns") was considered in Ref. [198], and Ref. [199] proposed a simple device to exploit these based on techniques of predictive coding (which is a special coding technique; see, e.g., Sec. 4.7.2 in Ref. [94]). In turn, Ref. [201] investigated a model where work extraction and information erasure are simultaneously possible (and are even enhanced by quantum correlations). It would also be interesting to investigate the role of temporal correlations in the unit string due to nonexponential waiting time distribution.

Another limitation of our results when deriving effective master equations is that, although system-unit interactions can be arbitrarily strong, the system-reservoir interaction must be weak and the resulting dynamics Markovian. Uzdin et al. [202] recently tried to tackle this issue via "heat exchangers" that could be strongly coupled to the system and that are equivalent to our units. Therefore, their work faces similar limitations as ours. Besides a few exact identities, the correct thermodynamic description of a system in strong contact with a continuous (perhaps non-Markovian) reservoir is still an open and active field of research [47,203-216].

As a final remark, let us note that connecting our present work to the recently developed quantum resource theories $[38,39]$ is an interesting perspective, as already indicated in Sec. III E. The goal of these theories is to establish an axiomatic mathematical framework to study quantum thermodynamics based on the study of the interconvertibility of states under certain constraints. In fact, if we switch off the permanent coupling to the heat reservoir, assume initially thermal units, and demand that $\Delta E_{S}+\Delta E_{U}=0$, our framework becomes identical to the one used in the resource theory of thermal operations [39]. However, we have demonstrated that a consistent thermodynamic framework can also be established for a 
much larger class of situations which are also of experimental relevance. Applying the tools and results from resource theory to such problems could prove useful.

\section{ACKNOWLEDGMENTS}

We thank Juan M. R. Parrondo for stimulating discussions concerning the statistical interpretation of the state of the units and its relation to entropy changes. This work benefited from the COST Action MP1209. Financial support by the DFG (Grants No. SCHA 1646/3-1, No. SFB 910, and No. GRK 1558), by the National Research Fund Luxembourg (Project No. FNR/A11/02), and by the European Research Council (Project No. 681456) is gratefully acknowledged.

Note added.-While this manuscript was under review, one of the co-authors, Tobias Brandes, passed away.

[1] R. Kosloff, Quantum Thermodynamics: A Dynamical Viewpoint, Entropy 15, 2100 (2013).

[2] G. Schaller, Open Quantum Systems Far from Equilibrium, Lecture Notes in Physics (Springer, New York, 2014).

[3] D. Gelbwaser-Klimovsky, W. Niedenzu, and G. Kurizki, Chapter Twelve-Thermodynamics of Quantum Systems under Dynamical Control, Adv. At. Mol. Opt. Phys. 64, 329 (2015).

[4] G. Bulnes Cuetara, M. Esposito, and G. Schaller, Quantum Thermodynamics with Degenerate Eigenstate Coherences, Entropy 18, 447 (2016).

[5] M. Esposito, U. Harbola, and S. Mukamel, Nonequilibrium Fluctuations, Fluctuation Theorems and Counting Statistics in Quantum Systems, Rev. Mod. Phys. 81, 1665 (2009).

[6] M. Campisi, P. Hänggi, and P. Talkner, Colloquium: Quantum Fluctuation Relations: Foundations and Applications, Rev. Mod. Phys. 83, 771 (2011).

[7] C. Jarzynski, Equalities and Inequalities: Irreversibility and the Second Law of Thermodynamics at the Nanoscale, Annu. Rev. Condens. Matter Phys. 2, 329 (2011).

[8] U. Seifert, Stochastic Thermodynamics, Fluctuation Theorems and Molecular Machines, Rep. Prog. Phys. 75, 126001 (2012).

[9] C. Van den Broeck and M. Esposito, Ensemble and Trajectory Thermodynamics: A Brief Introduction, Physica (Amsterdam) 418A, 6 (2015).

[10] C. Bustamante, J. Liphardt, and F. Ritort, The Nonequilibrium Thermodynamics of Small Systems, Phys. Today 58, 43 (2005).

[11] H. Ge, M. Qian, and H. Qian, Stochastic Theory of Nonequilibrium Steady States. Part II: Applications in Chemical Biophysics, Phys. Rep. 510, 87 (2012).

[12] S. Ciliberto, R. Gomez-Solano, and A. Petrosyan, Fluctuations, Linear Response and Currents in Out-ofEquilibrium Systems, Annu. Rev. Condens. Matter Phys. 4, 235 (2013).
[13] J.P. Pekola, Towards Quantum Thermodynamics in Electronic Circuits, Nat. Phys. 11, 118 (2015).

[14] H. Thierschmann, R. Sánchez, B. Sothmann, H. Buhmann, and L. W. Molenkamp, Thermoelectrics with Coulomb Coupled Quantum Dots, C.R. Phys. 17, 1109 (2016).

[15] M. Serra-Garcia, A. Foehr, M. Molerón, J. Lydon, C. Chong, and C. Daraio, Mechanical Autonomous Stochastic Heat Engine, Phys. Rev. Lett. 117, 010602 (2016).

[16] J. Roßnagel, S. T. Dawkins, K. N. Tolazzi, O. Abah, E. Lutz, F. Schmidt-Kaler, and K. Singer, A Single-Atom Heat Engine, Science 352, 325 (2016).

[17] C. H. Bennett, The Thermodynamics of Computation-A Review, Int. J. Theor. Phys. 21, 905 (1982).

[18] Maxwell's Demon 2: Entropy, Classical and Quantum Information, Computing, edited by H. S. Leff and A. F. Rex (IOP Publishing, Bristol, 2003).

[19] K. Maruyama, F. Nori, and V. Vedral, Colloquium: The Physics of Maxwell's Demon and Information, Rev. Mod. Phys. 81, 1 (2009).

[20] T. Sagawa, Thermodynamic and Logical Reversibilities Revisited, J. Stat. Mech. (2014) P03025.

[21] J. M. R. Parrondo, J. M. Horowitz, and T. Sagawa, Thermodynamics of Information, Nat. Phys. 11, 131 (2015).

[22] M. O. Scully, M.S. Zubairy, G. S. Agarwal, and H. Walther, Extracting Work from a Single Heat Bath via Vanishing Quantum Coherence, Science 299, 862 (2003).

[23] R. Dillenschneider and E. Lutz, Energetics of Quantum Correlations, Europhys. Lett. 88, 50003 (2009).

[24] J. Roßnagel, O. Abah, F. Schmidt-Kaler, K. Singer, and E. Lutz, Nanoscale Heat Engine Beyond the Carnot Limit, Phys. Rev. Lett. 112, 030602 (2014).

[25] H. Li, J. Zou, W.-L. Yu, B.-M. Xu, J.-G. Li, and B. Shao, Quantum Coherence Rather than Quantum Correlations Reflect the Effects of a Reservoir on a System's Work Capability, Phys. Rev. E 89, 052132 (2014).

[26] R. Alicki and D. Gelbwaser-Klimovsky, Non-equilibrium Quantum Heat Machines, New J. Phys. 17, 115012 (2015).

[27] G. Manzano, F. Galve, R. Zambrini, and J. M. R. Parrondo, Entropy Production and Thermodynamic Power of the Squeezed Thermal Reservoir, Phys. Rev. E 93, 052120 (2016).

[28] W. Niedenzu, A. G. Kofman, D. Gelbwaser-Klimovsky, and G. Kurizki, On the Operation of Machines Powered by Quantum Non-thermal Baths, New J. Phys. 18, 083012 (2016).

[29] B. Gardas and S. Deffner, Thermodynamic Universality of Quantum Carnot Engines, Phys. Rev. E 92, 042126 (2015).

[30] B. Gardas, S. Deffner, and A. Saxena, Non-Hermitian Quantum Thermodynamics, Sci. Rep. 6, 23408 (2016).

[31] D. Meschede, H. Walther, and G. Müller, One-Atom Maser, Phys. Rev. Lett. 54, 551 (1985).

[32] P. Filipowicz, J. Javanainen, and P. Meystre, Theory of a Microscopic Maser, Phys. Rev. A 34, 3077 (1986).

[33] M. O. Scully and M. Suhail Zubairy, Quantum Optics (Cambridge University Press, Cambridge, England, 1997).

[34] C. Sayrin, I. Dotsenko, X. Zhou, B. Peaudecerf, T. Rybarczyk, S. Gleyzes, P. Rouchon, M. Mirrahimi, H. Amini, M. Brune, J.-M. Raimond, and S. Haroche, 
Real-Time Quantum Feedback Prepares and Stabilizes Photon Number States, Nature (London) 477, 73 (2011).

[35] D. Mandal and C. Jarzynski, Work and Information Processing in a Solvable Model of Maxwell's Demon, Proc. Natl. Acad. Sci. U.S.A. 109, 11641 (2012).

[36] D. Mandal, H. T. Quan, and C. Jarzynski, Maxwell's Refrigerator: An Exactly Solvable Model, Phys. Rev. Lett. 111, 030602 (2013).

[37] P. Strasberg, J. Cerrillo, G. Schaller, and T. Brandes, Thermodynamics of Stochastic Turing Machines, Phys. Rev. E 92, 042104 (2015).

[38] G. Gour, M. P. Müller, V. Narasimhachar, R. W. Spekkens, and N. Y. Halpern, The Resource Theory of Informational Nonequilibrium in Thermodynamics, Phys. Rep. 583, 1 (2015).

[39] J. Goold, M. Huber, A. Riera, L. del Rio, and P. Skrzypzyk, The Role of Quantum Information in Thermodynamics-A Topical Review, J. Phys. A 49, 143001 (2016).

[40] R. Uzdin and R. Kosloff, The Multilevel Four-Stroke Swap Engine and Its Environment, New J. Phys. 16, 095003 (2014).

[41] R. S. Whitney, Most Efficient Quantum Thermoelectric at Finite Power Output, Phys. Rev. Lett. 112, 130601 (2014).

[42] K. Kraus, States, Effects and Operations: Fundamental Notions of Quantum Theory (Springer-Verlag, Berlin, Heidelberg, 1983).

[43] M. A. Nielsen and I. L. Chuang, Quantum Computation and Quantum Information (Cambridge University Press, Cambridge, England, 2000).

[44] V. Vedral, The Role of Relative Entropy in Quantum Information Theory, Rev. Mod. Phys. 74, 197 (2002).

[45] H. M. Wiseman and G. J. Milburn, Quantum Measurement and Control (Cambridge University Press, Cambridge, England, 2010).

[46] G. Lindblad, Completely Positive Maps and Entropy Inequalities, Commun. Math. Phys. 40, 147 (1975).

[47] M. Esposito, K. Lindenberg, and C. Van den Broeck, Entropy Production as Correlation Between System and Reservoir, New J. Phys. 12, 013013 (2010).

[48] S. Deffner and C. Jarzynski, Information Processing and the Second Law of Thermodynamics: An Inclusive, Hamiltonian Approach, Phys. Rev. X 3, 041003 (2013).

[49] D. Reeb and M. M. Wolf, An Improved Landauer Principle with Finite-Size Corrections, New J. Phys. 16, 103011 (2014).

[50] J. Goold, M. Paternostro, and K. Modi, Nonequilibrium Quantum Landauer Principle, Phys. Rev. Lett. 114, 060602 (2015).

[51] D. Kondepudi and I. Prigogine, Modern Thermodynamics: From Heat Engines to Dissipative Structures (John Wiley \& Sons, West Sussex, 2007).

[52] Proving $D\left[\rho_{R}(\tau) \| \rho_{\beta}^{R}\right]=\mathcal{O}\left(\epsilon^{2}\right)$ can be done by expanding in a power series $D\left[\rho_{R}(\tau) \| \rho_{\beta}^{R}\right]=D_{0}+\epsilon D_{1}+\mathcal{O}\left(\epsilon^{2}\right)$. Clearly, $D_{0}=D\left[\rho_{\beta}^{R} \| \rho_{\beta}^{R}\right]=0$ and $D_{1}=0$ due to Klein's inequality (10) because $\epsilon$ can be positive as well as negative.

[53] B. Gaveau and L. S. Schulman, A General Framework for Non-equilibrium Phenomena: The Master Equation and Its Formal Consequences, Phys. Lett. A 229, 347 (1997).
[54] G. E. Crooks, Beyond Boltzmann-Gibbs Statistics: Maximum Entropy Hyperensembles out of Equilibrium, Phys. Rev. E 75, 041119 (2007).

[55] M. Esposito and C. Van den Broeck, Second Law and Landauer Principle Far from Equilibrium, Europhys. Lett. 95, 40004 (2011).

[56] K. Jacobs, Second Law of Thermodynamics and Quantum Feedback Control: Maxwell's Demon with Weak Measurements, Phys. Rev. A 80, 012322 (2009).

[57] J. M. Horowitz and J. M. R. Parrondo, Designing Optimal Discrete-Feedback Thermodynamic Engines, New J. Phys. 13, 123019 (2011).

[58] P. Skrzypczyk, A. J. Short, and S. Popescu, Work Extraction and Thermodynamics for Individual Quantum Systems, Nat. Commun. 5, 4185 (2014).

[59] H. Spohn and J. L. Lebowitz, Irreversible Thermodynamics for Quantum Systems Weakly Coupled to Thermal Reservoirs, Adv. Chem. Phys. 38, 109 (1979).

[60] R. Alicki, The Quantum Open System as a Model of the Heat Engine, J. Phys. A 12, L103 (1979).

[61] H. P. Breuer and F. Petruccione, The Theory of Open Quantum Systems (Oxford University Press, Oxford, 2002).

[62] As a technical side remark, one should note that the system Hamiltonian $H_{X}(t)$ usually gets renormalized due to Lamb shift terms [61]. In the following, we will not explicitly mention them, tacitly assuming that they were already conveniently absorbed in the definition of $H_{X}(t)$.

[63] F. Haupt, M. Leijnse, H. L. Calvo, L. Classen, J. Splettstoesser, and M.R. Wegewijs, Heat, Molecular Vibrations and Adiabatic Driving in Nonequilibrium Transport through Interacting Quantum Dots, Phys. Status Solidi B 250, 2315 (2013).

[64] M. Yamaguchi, T. Yuge, and T. Ogawa, Markovian Quantum Master Equation Beyond Adiabatic Regime, Phys. Rev. E 95, 012136 (2017).

[65] Other MEs can be derived for (strong) periodic driving using Floquet theory $[1,3,61]$ and also give rise to a consistent nonequilibrium thermodynamics [1,3,66,67]. If the driving is neither slow nor periodic, no method is currently known to derive a ME, although the thermodynamic quantities defined here remain meaningful; see also Ref. [68].

[66] K. Szczygielski, D. Gelbwaser-Klimovsky, and R. Alicki, Markovian Master Equation and Thermodynamics of a Two-Level System in a Strong Laser Field, Phys. Rev. E 87, 012120 (2013).

[67] G. Bulnes Cuetara, A. Engel, and M. Esposito, Stochastic Thermodynamics of Rapidly Driven Quantum Systems, New J. Phys. 17, 055002 (2015).

[68] S. Deffner and E. Lutz, Nonequilibrium Entropy Production for Open Quantum Systems, Phys. Rev. Lett. 107, 140404 (2011).

[69] G. Lindblad, On the Generators of Quantum Dynamical Semigroups, Commun. Math. Phys. 48, 119 (1976).

[70] V. Gorini, A. Kossakowski, and E. C. G. Sudarshan, Completely Positive Dynamical Semigroups of $N$-Level Systems, J. Math. Phys. (N.Y.) 17, 821 (1976).

[71] H. Spohn, Entropy Production for Quantum Dynamical Semigroups, J. Math. Phys. (N.Y.) 19, 1227 (1978). 
[72] In Sec. V C, we discuss, for a particular application, which changes have to be made if this assumption is not fulfilled.

[73] In fact, if the $m$ th unit is allowed to come back and interact again with the system in the $n$th interval $(n>m)$, then a simple description in terms of the system and single unit (as carried out above) would not be possible anymore.

[74] The proof is easily carried out by first noting that any system state must asymptotically (i.e., for $n \rightarrow \infty$ ) reach some steady state because the sequence $f_{n} \equiv$ $D\left[\Phi_{S}^{n}\left(\rho_{S}\right) \| \sigma_{S}\right]$ for some steady state $\sigma_{S}$ is monotonically decreasing and bounded from below, and second, by realizing that the existence of two different steady states $\sigma_{S}$ and $\sigma_{S}^{*}$ contradicts Eq. (54).

[75] D. Petz, Monotonicity of Quantum Relative Entropy Revisited, Rev. Math. Phys. 15, 79 (2003).

[76] M. Horodecki and J. Oppenheim, Fundamental Limitations for Quantum and Nanoscale Thermodynamics, Nat. Commun. 4, 2059 (2013).

[77] F. Brandão, M. Horodecki, N. Ng, J. Oppenheim, and S. Wehner, The Second Laws of Quantum Thermodynamics, Proc. Natl. Acad. Sci. U.S.A. 112, 3275 (2015).

[78] M. Lostaglio, D. Jennings, and T. Rudolph, Description of Quantum Coherence in Thermodynamic Processes Requires Constraints Beyond Free Energy, Nat. Commun. 6, 6383 (2015).

[79] D. E. Bruschi, M. Perarnau-Llobet, N. Friis, K. V. Hovhannisyan, and M. Huber, Thermodynamics of Creating Correlations: Limitations and Optimal Protocols, Phys. Rev. E 91, 032118 (2015).

[80] M.P. Woods, N. Ng, and S. Wehner, The Maximum Efficiency of Nano Heat Engines Depends on More than Temperature, arXiv:1506.02322 [Phys. Rev. X (to be published)].

[81] H. Wilming, R. Gallego, and J. Eisert, Second Law of Thermodynamics under Control Restrictions, Phys. Rev. E 93, 042126 (2016).

[82] K. Korzekwa, M. Lostaglio, J. Oppenheim, and D. Jennings, The Extraction of Work from Quantum Coherence, New J. Phys. 18, 023045 (2016).

[83] To quote Sommerfeld (p. 36 in Ref. [84]): “Thermodynamics investigates the conditions that govern the transformation of heat into work. It teaches us to recognize temperature as the measure of the work-value of heat. Heat of higher temperature is richer, is capable of doing more work. Work may be regarded as heat of an infinitely high temperature, as unconditionally available heat."

[84] A. Sommerfeld, Atomic Structure and Spectral Lines (Methuen \& Co, London, 1923).

[85] A. E. Allahverdyan and T. M. Nieuwenhuizen, Fluctuations of Work from Quantum Subensembles: The Case Against Quantum Work-Fluctuation Theorems, Phys. Rev. E 71, 066102 (2005).

[86] P. Talkner, E. Lutz, and P. Hänggi, Fluctuation Theorems: Work Is Not an Observable, Phys. Rev. E 75, 050102(R) (2007).

[87] H. Weimer, M. J. Henrich, F. Rempp, H. Schröder, and G. Mahler, Local Effective Dynamics of Quantum Systems: A Generalized Approach to Work and Heat, Europhys. Lett. 83, 30008 (2008).
[88] H. Schröder and G. Mahler, Work Exchange Between Quantum Systems: The Spin-Oscillator Model, Phys. Rev. E 81, 021118 (2010).

[89] J. Salmilehto, P. Solinas, and M. Möttönen, Quantum Driving and Work, Phys. Rev. E 89, 052128 (2014).

[90] H. Hossein-Nejad, E. J. O'Reilly, and A. Olaya-Castro, Work, Heat and Entropy Production in Bipartite Quantum Systems, New J. Phys. 17, 075014 (2015).

[91] R. Gallego, J. Eisert, and H. Wilming, Thermodynamic Work from Operational Principles, New J. Phys. 18, 103017 (2016).

[92] C. Jarzynski, H. T. Quan, and S. Rahav, The QuantumClassical Correspondence Principle for Work Distributions, Phys. Rev. X 5, 031038 (2015).

[93] C. E. Shannon, A Mathematical Theory of Communication, Bell Syst. Tech. J. 27, 379 (1948).

[94] R. P. Feynman, Feynman Lectures on Computation (Addison-Wesley Publishing Company, Reading, 1996).

[95] J. Hoppenau and A. Engel, On the Energetics of Information Exchange, Europhys. Lett. 105, 50002 (2014).

[96] M. Esposito, Stochastic Thermodynamics under Coarse Graining, Phys. Rev. E 85, 041125 (2012).

[97] M. O. Scully, Extracting Work from a Single Thermal Bath via Quantum Negentropy, Phys. Rev. Lett. 87, 220601 (2001).

[98] A. C. Barato and U. Seifert, An Autonomous and Reversible Maxwell's Demon, Europhys. Lett. 101, 60001 (2013).

[99] S. Deffner, Information-Driven Current in a Quantum Maxwell Demon, Phys. Rev. E 88, 062128 (2013).

[100] Z. Lu, D. Mandal, and C. Jarzynski, Engineering Maxwell's Demon, Phys. Today 67, 60 (2014).

[101] A. C. Barato and U. Seifert, Stochastic Thermodynamics with Information Reservoirs, Phys. Rev. E 90, 042150 (2014).

[102] P. Strasberg, G. Schaller, T. Brandes, and C. Jarzynski, The Second Laws for an Information Driven Current through a Spin Valve, Phys. Rev. E 90, 062107 (2014).

[103] Y. Cao, Z. Gong, and H. T. Quan, Thermodynamics of Information Processing Based on Enzyme Kinetics: An Exactly Solvable Model of an Information Pump, Phys. Rev. E 91, 062117 (2015).

[104] R. Landauer, Irreversibility and Heat Generation in the Computing Process, IBM J. Res. Dev. 5, 183 (1961).

[105] R. W. Keyes and R. Landauer, Minimal Energy Dissipation in Logic, IBM J. Res. Dev. 14, 152 (1970).

[106] K. Likharev, Classical and Quantum Limitations on Energy Consumption in Computation, Int. J. Theor. Phys. 21, 311 (1982).

[107] K. Shizume, Heat Generation Required by Information Erasure, Phys. Rev. E 52, 3495 (1995).

[108] B. Piechocinska, Information Erasure, Phys. Rev. A 61, 062314 (2000).

[109] M. B. Plenio and V. Vitelli, The Physics of Forgetting: Landauer's Erasure Principle and Information Theory, Contemp. Phys. 42, 25 (2001).

[110] T. Sagawa and M. Ueda, Minimal Energy Cost for Thermodynamic Information Processing: Measurement and Information Erasure, Phys. Rev. Lett. 102, 250602 (2009). 
[111] E. Aurell, K. Gawedzki, C. Mejía-Monasterio, R. Mohayaee, and P. Muratore-Ginanneschi, Refined Second Law of Thermodynamics for Fast Random Processes, J. Stat. Phys. 147, 487 (2012).

[112] G. Diana, G. B. Bagci, and M. Esposito, Finite-Time Erasing of Information Stored in Fermionic Bits, Phys. Rev. E 87, 012111 (2013).

[113] S. Lorenzo, R. McCloskey, F. Ciccarello, M. Paternostro, and G. M. Palma, Landauer's Principle in Multipartite Open Quantum System Dynamics, Phys. Rev. Lett. 115, 120403 (2015).

[114] E. Hanson, A. Joye, Y. Pautrat, and R. Raquépas, Landauer's Principle in Repeated Interaction Systems, Commun. Math. Phys. 349, 285 (2017).

[115] M. Pezzutto, M. Paternostro, and Y. Omar, Implications of Non-Markovian Dynamics for the Landauer Bound, New J. Phys. 18, 123018 (2016).

[116] A. O. Orlov, C. S. Lent, C. C. Thorpe, G. P. Boechler, and G. L. Snider, Experimental Test of Landauer's Principle at the Sub- $k_{b} T$ Level, Jpn. J. Appl. Phys. 51, 06FE10 (2012).

[117] A. Bérut, A. Arakelyan, A. Petrosyan, S. Ciliberto, R. Dillenschneider, and E. Lutz, Experimental Verification of Landauer's Principle Linking Information and Thermodynamics, Nature (London) 483, 187 (2012).

[118] Y. Jun, M. Gavrilov, and J. Bechhoefer, High-Precision Test of Landauer's Principle in a Feedback Trap, Phys. Rev. Lett. 113, 190601 (2014).

[119] A. Bérut, A. Petrosyan, and S. Ciliberto, Information and Thermodynamics: Experimental Verification of Landauer's Erasure Principle, J. Stat. Mech. (2015) P06015.

[120] J. P. P. Silva, R. S. Sarthour, A. M. Souza, I. S. Oliveira, J. Goold, K. Modi, D. O. Soares-Pinto, and L. C. Céleri, Experimental Demonstration of Information to Energy Conversion in a Quantum System at the Landauer Limit, Proc. R. Soc. A 472, 20150813 (2016).

[121] J. Hong, B. Lambson, S. Dhuey, and J. Bokor, Experimental Test of Landauer's Principle in Single-Bit Operations on Nanomagnetic Memory Bits, Sci. Adv. 2, e1501492 (2016).

[122] M. Gavrilov and J. Bechhoefer, Erasure without Work in an Asymmetric Double-Well Potential, Phys. Rev. Lett. 117, 200601 (2016).

[123] J.D. Norton, All Shook up: Fluctuations, Maxwell's Demon and the Thermodynamics of Computation, Entropy 15, 4432 (2013).

[124] L. Del Rio, J. Åberg, R. Renner, O. Dahlsten, and V. Vedral, The Thermodynamic Meaning of Negative Entropy, Nature (London) 474, 61 (2011).

[125] This statement is also true in the classical context and does not require any quantum entanglement.

[126] If randomization was a computational process, one could build computers that are perfect random number generators, which is not the case.

[127] S. Lloyd, Use of Mutual Information to Decrease Entropy: Implications for the Second Law of Thermodynamics, Phys. Rev. A 39, 5378 (1989).

[128] H. Touchette and S. Lloyd, Information-Theoretic Limits of Control, Phys. Rev. Lett. 84, 1156 (2000).
[129] H. Touchette and S. Lloyd, Information-Theoretic Approach to the Study of Control Systems, Physica (Amsterdam) 331A, 140 (2004).

[130] T. Sagawa and M. Ueda, Second Law of Thermodynamics with Discrete Quantum Feedback Control, Phys. Rev. Lett. 100, 080403 (2008).

[131] F. J. Cao and M. Feito, Thermodynamics of Feedback Controlled Systems, Phys. Rev. E 79, 041118 (2009).

[132] T. Sagawa and M. Ueda, Generalized Jarzynski Equality under Nonequilibrium Feedback Control, Phys. Rev. Lett. 104, 090602 (2010).

[133] S. Deffner and E. Lutz, Information Free Energy for Nonequilibrium States, arXiv:1201.3888 [Phys. Rev. Lett. (to be published)].

[134] M. Ponmurugan, Generalized Detailed Fluctuation Theorem under Nonequilibrium Feedback Control, Phys. Rev. E 82, 031129 (2010).

[135] J. M. Horowitz and S. Vaikuntanathan, Nonequilibrium Detailed Fluctuation Theorem for Repeated Discrete Feedback, Phys. Rev. E 82, 061120 (2010).

[136] D. Abreu and U. Seifert, Thermodynamics of Genuine Nonequilibrium States under Feedback Control, Phys. Rev. Lett. 108, 030601 (2012).

[137] T. Sagawa and M. Ueda, Fluctuation Theorem with Information Exchange: Role of Correlations in Stochastic Thermodynamics, Phys. Rev. Lett. 109, 180602 (2012).

[138] T. Sagawa and M. Ueda, Nonequilibrium Thermodynamics of Feedback Control, Phys. Rev. E 85, 021104 (2012).

[139] S. Toyabe, T. Sagawa, M. Ueda, E. Muneyuki, and M. Sano, Experimental Demonstration of Information-toEnergy Conversion and Validation of the Generalized Jarzynski Equality, Nat. Phys. 6, 988 (2010).

[140] J. V. Koski, V. F. Maisi, J. P. Pekola, and D. V. Averin, Experimental Realization of a Szilard Engine with a Single Electron, Proc. Natl. Acad. Sci. U.S.A. 111, 13786 (2014).

[141] J. V. Koski, V. F. Maisi, T. Sagawa, and J. P. Pekola, Experimental Observation of the Role of Mutual Information in the Nonequilibrium Dynamics of a Maxwell Demon, Phys. Rev. Lett. 113, 030601 (2014).

[142] The unitary evolution operator associated with $H_{\mathrm{fb}}(t)$ is then of the form $U_{\mathrm{fb}}=\sum_{o} \Pi_{o} U_{S R}^{(o)}$, where $U_{S R}^{(o)}$ acts exclusively in the system-reservoir space and the reduced system-reservoir state is obtained by tracing over the unit Hilbert space.

[143] H. M. Wiseman, Ph.D. thesis, University of Queensland, 1994.

[144] H. M. Wiseman and G. J. Milburn, All-Optical Versus Electro-optical Feedback, Phys. Rev. A 49, 4110 (1994).

[145] S. Lloyd, Coherent Quantum Feedback, Phys. Rev. A 62, 022108 (2000).

[146] J. Bechhoefer, Feedback for Physicists: A Tutorial Essay on Control, Rev. Mod. Phys. 77, 783 (2005).

[147] We note that Eq. (75) does not rely on the assumption that the system-unit state is decorrelated at the beginning of the feedback interval [see Eq. (20) or (35)]. This assumption is only used initially (before the measurement), and it enables us to consider a regime of repeated interactions.

[148] A. C. Barato and U. Seifert, Unifying Three Perspectives on Information Processing in Stochastic Thermodynamics, Phys. Rev. Lett. 112, 090601 (2014). 
[149] J. M. Horowitz and H. Sandberg, Second-Law-Like Inequalities with Information and Their Interpretations, New J. Phys. 16, 125007 (2014).

[150] N. Shiraishi, T. Matsumoto, and T. Sagawa, MeasurementFeedback Formalism Meets Information Reservoirs, New J. Phys. 18, 013044 (2016).

[151] M. Esposito and G. Schaller, Stochastic Thermodynamics for “Maxwell Demon" Feedbacks, Europhys. Lett. 99, 30003 (2012).

[152] T. Munakata and M. L. Rosinberg, Entropy Production and Fluctuation Theorems under Feedback Control: The Molecular Tefrigerator Model Revisited, J. Stat. Mech. (2012) P05010.

[153] P. Strasberg, G. Schaller, T. Brandes, and M. Esposito, Thermodynamics of Quantum-Jump-Conditioned Feedback Control, Phys. Rev. E 88, 062107 (2013).

[154] T. Munakata and M. L. Rosinberg, Feedback Cooling, Measurement Errors and Entropy Production, J. Stat. Mech. (2013) P06014.

[155] P. Strasberg, G. Schaller, T. Brandes, and M. Esposito, Thermodynamics of a Physical Model Implementing a Maxwell Demon, Phys. Rev. Lett. 110, 040601 (2013).

[156] D. Hartich, A. C. Barato, and U. Seifert, Stochastic Thermodynamics of Bipartite Systems: Transfer Entropy Inequalities and a Maxwell's Demon Interpretation, J. Stat. Mech. (2014) P02016.

[157] J. M. Horowitz and M. Esposito, Thermodynamics with Continuous Information Flow, Phys. Rev. X 4, 031015 (2014).

[158] N. Shiraishi and T. Sagawa, Fluctuation Theorem for Partially Masked Nonequilibrium Dynamics, Phys. Rev. E 91, 012130 (2015).

[159] J. M. Horowitz, Multipartite Information Flow for Multiple Maxwell Demons, J. Stat. Mech. (2015) P03006.

[160] J. M. Horowitz, Quantum-Trajectory Approach to the Stochastic Thermodynamics of a Forced Harmonic Oscillator, Phys. Rev. E 85, 031110 (2012).

[161] J. M. Horowitz and J. M. R. Parrondo, Entropy Production Along Nonequilibrium Quantum Jump Trajectories, New J. Phys. 15, 085028 (2013).

[162] F. Barra, The Thermodynamic Cost of Driving Quantum Systems by Their Boundaries, Sci. Rep. 5, 14873 (2015).

[163] S. Lorenzo, A. Farace, F. Ciccarello, G. Massimo Palma, and V. Giovannetti, Heat Flux and Quantum Correlations in Dissipative Cascaded Systems, Phys. Rev. A 91, 022121 (2015).

[164] P. Filipowicz, J. Javanainen, and P. Meystre, Quantum and Semiclassical Steady States of a Kicked Cavity Mode, J. Opt. Soc. Am. B 3, 906 (1986).

[165] G. J. Milburn, Kicked Quantized Cavity Mode: An OpenSystems-Theory Approach, Phys. Rev. A 36, 744 (1987).

[166] S. Attal and Y. Pautrat, From Repeated to Continuous Quantum Interactions, Ann. Inst. Henri Poincaré 7, 59 (2006).

[167] D. Grimmer, D. Layden, R. B. Mann, and E. MartínMartínez, Open Dynamics under Rapid Repeated Interaction, Phys. Rev. A 94, 032126 (2016).

[168] D. Karevski and T. Platini, Quantum Nonequilibrium Steady States Induced by Repeated Interactions, Phys. Rev. Lett. 102, 207207 (2009).
[169] V. Giovannetti and G. M. Palma, Master Equations for Correlated Quantum Channels, Phys. Rev. Lett. 108, 040401 (2012).

[170] J. Åberg, Catalytic coherence, Phys. Rev. Lett. 113, 150402 (2014).

[171] A. S. L. Malabarba, A. J. Short, and P. Kammerlander, Clock-Driven Quantum Thermal Engines, New J. Phys. 17, 045027 (2015).

[172] In this case, each unit in our setup would actually correspond to two subunits, which are both pairwise entangled with each other but interact with the system sequentially.

[173] D. A. Rodrigues, J. Imbers, and A. D. Armour, Quantum Dynamics of a Resonator Driven by a Superconducting Single-Electron Transistor: A Solid-State Analogue of the Micromaser, Phys. Rev. Lett. 98, 067204 (2007).

[174] G. Rempe, F. Schmidt-Kaler, and H. Walther, Observation of Sub-Poissonian Photon Statistics in a Micromaser, Phys. Rev. Lett. 64, 2783 (1990).

[175] M. O. Scully, Quantum Photocell: Using Quantum Coherence to Reduce Radiative Recombination and Increase Efficiency, Phys. Rev. Lett. 104, 207701 (2010).

[176] M. O. Scully, K. R. Chapin, K. E. Dorfman, M. B. Kim, and A. Svidzinsky, Quantum Heat Engine Power Can Be Increased by Noise-Induced Coherence, Proc. Natl. Acad. Sci. U.S.A. 108, 15097 (2011).

[177] S. Datta, Nanodevices and Maxwells Demon, in Nanoscale Phenomena (Springer, New York, 2008), pp. 59-81.

[178] G. Schaller, C. Emary, G. Kiesslich, and T. Brandes, Probing the Power of an Electronic Maxwell's Demon: Single-Electron Transistor Monitored by a Quantum Point Contact, Phys. Rev. B 84, 085418 (2011).

[179] D. V. Averin, M. Möttönen, and J. P. Pekola, Maxwell's Demon Based on a Single-Electron Pump, Phys. Rev. B 84, 245448 (2011).

[180] L. B. Kish and C.-G. Granqvist, Electrical Maxwell Demon and Szilard Engine Utilizing Johnson Noise, Measurement, Logic and Control, PLoS One 7, e46800 (2012).

[181] J. Bergli, Y. M. Galperin, and N. B. Kopnin, Information Flow and Optimal Protocol for a Maxwell-Demon SingleElectron Pump, Phys. Rev. E 88, 062139 (2013).

[182] A. Kutvonen, J. Koski, and T. Ala-Nissilä, Thermodynamics and Efficiency of an Autonomous On-Chip Maxwell's Demon, Sci. Rep. 6, 21126 (2016).

[183] J. V. Koski, A. Kutvonen, I. M. Khaymovich, T. Ala-Nissila, and J. P. Pekola, On-Chip Maxwell's Demon as an Information-Powered Refrigerator, Phys. Rev. Lett. 115, 260602 (2015).

[184] G. Schaller, Fighting Decoherence by FeedbackControlled Dissipation, Phys. Rev. A 85, 062118 (2012).

[185] R. Uzdin, Coherence-Induced Reversibility and Collective Operation of Quantum Heat Machines via Coherence Recycling, Phys. Rev. Applied 6, 024004 (2016).

[186] P. Kammerlander and J. Anders, Coherence and Measurement in Quantum Thermodynamics, Sci. Rep. 6, 22174 (2016).

[187] We note that the maximum value of the quantum mutual information $I_{S: U}$ can be twice as large as its classical counterpart. Thus, quantum correlations in the outgoing 
state have the potential to degrade the amount of extractable work to a larger extent than classical ones.

[188] If the highest energy belongs to a degenerate subspace, i.e., $E_{N}=E_{N-1}=\ldots$, then there is an additional freedom in the choice of $\rho_{U}(0)$ which, however, does not change the nonequilibrium free energy.

[189] We remark that the argument presented here is even valid at finite times $\tau<\infty$ and for time-dependent Hamiltonians, i.e., out of equilibrium. Realizing that a Gibbs state is a state of minimum free energy with respect to a reservoir at inverse temperature $\beta$ is therefore of no help since the system might be driven and/or does not have time to reach the Gibbs state.

[190] K. V. Hovhannisyan, M. Perarnau-Llobet, M. Huber, and A. Acín, Entanglement Generation Is Not Necessary for Optimal Work Extraction, Phys. Rev. Lett. 111, 240401 (2013).

[191] N. Brunner, M. Huber, N. Linden, S. Popescu, R. Silva, and P. Skrzypczyk, Entanglement Enhances Cooling in Microscopic Quantum Refrigerators, Phys. Rev. E 89, 032115 (2014).

[192] F. C. Binder, S. Vinjanampathy, K. Modi, and J. Goold, Quantacell: Powerful Charging of Quantum Batteries, New J. Phys. 17, 075015 (2015).

[193] R. Uzdin, A. Levy, and R. Kosloff, Equivalence of Quantum Heat Machines, and Quantum-Thermodynamic Signatures, Phys. Rev. X 5, 031044 (2015).

[194] W. Niedenzu, D. Gelbwaser-Klimovsky, and G. Kurizki, Performance Limits of Multilevel and Multipartite Quantum Heat Machines, Phys. Rev. E 92, 042123 (2015).

[195] D. Gelbwaser-Klimovsky, W. Niedenzu, P. Brumer, and G. Kurizki, Power Enhancement of Heat Engines via Correlated Thermalization in a Three-Level "Working Fluid", Sci. Rep. 5, 14413 (2015).

[196] N. Merhav, Sequence Complexity and Work Extraction, J. Stat. Mech. (2015) P06037.

[197] A. B. Boyd, D. Mandal, and J. P. Crutchfield, Identifying Functional Thermodynamics in Autonomous Maxwellian Ratchets, New J. Phys. 18, 023049 (2016).

[198] A. J. Garner, J. Thompson, V. Vedral, and M. Gu, The Thermodynamics of Complexity and Pattern Manipulation, arXiv:1510.00010 [Phys. Rev. X (to be published)].

[199] P. Strasberg, Ph.D. thesis, TU Berlin, 2015.

[200] A. B. Boyd, D. Mandal, and J. P. Crutchfield, CorrelationPowered Information Engines and the Thermodynamics of Self-Correction, Phys. Rev. E 95, 012152 (2017).

[201] A. Chapman and A. Miyake, How Can an Autonomous Quantum Maxwell Demon Harness Correlated Information?, Phys. Rev. E 92, 062125 (2015).
[202] R. Uzdin, A. Levy, and R. Kosloff, Quantum Heat Machines Equivalence, Work Extraction Beyond Markovianity and Strong Coupling via Heat Exchangers, Entropy 18, 124 (2016).

[203] C. Jarzynski, Nonequilibrium Work Theorem for a System Strongly Coupled to a Thermal Environment, J. Stat. Mech. (2004) P09005.

[204] M. Campisi, P. Talkner, and P. Hänggi, Fluctuation Theorem for Arbitrary Open Quantum Systems, Phys. Rev. Lett. 102, 210401 (2009).

[205] G. Schaller, T. Krause, T. Brandes, and M. Esposito, Single-Electron Transistor Strongly Coupled to Vibrations: Counting Statistics and Fluctuation Theorem, New J. Phys. 15, 033032 (2013).

[206] L. Pucci, M. Esposito, and L. Peliti, Entropy Production in Quantum Brownian Motion, J. Stat. Mech. (2013) P04005.

[207] M. F. Ludovico, J. S. Lim, M. Moskalets, L. Arrachea, and D. Sánchez, Dynamical Energy Transfer in ac-Driven Quantum Systems, Phys. Rev. B 89, 161306 (2014).

[208] M. Esposito, M. A. Ochoa, and M. Galperin, Quantum Thermodynamics: A Nonequilibrium Greens Function Approach, Phys. Rev. Lett. 114, 080602 (2015).

[209] M. Esposito, M. A. Ochoa, and M. Galperin, Nature of Heat in Strongly Coupled Open Quantum Systems, Phys. Rev. B 92, 235440 (2015).

[210] A. Bruch, M. Thomas, S. V. Kusminskiy, F. von Oppen, and A. Nitzan, Quantum Thermodynamics of the Driven Resonant Level Model, Phys. Rev. B 93, 115318 (2016).

[211] U. Seifert, First and Second Law of Thermodynamics at Strong Coupling, Phys. Rev. Lett. 116, 020601 (2016).

[212] P. Strasberg, G. Schaller, N. Lambert, and T. Brandes, Nonequilibrium Thermodynamics in the Strong Coupling and Non-Markovian Regime Based on a Reaction Coordinate Mapping, New J. Phys. 18, 073007 (2016).

[213] M. Carrega, P. Solinas, M. Sassetti, and U. Weiss, Energy Exchange in Driven Open Quantum Systems at Strong Coupling, Phys. Rev. Lett. 116, 240403 (2016).

[214] P. Talkner and P. Hänggi, Open System Trajectories Specify Fluctuating Work but Not Heat, Phys. Rev. E 94, 022143 (2016).

[215] A. Kato and Y. Tanimura, Quantum Heat Current under Non-perturbative and Non-Markovian Conditions: Applications to Heat Machines, J. Chem. Phys. 145, 224105 (2016).

[216] C. Jarzynski, Stochastic and Macroscopic Thermodynamics of Strongly Coupled Systems, Phys. Rev. X 7, 011008 (2017). 